\title{
Four-Manifolds with Positive Isotropic Curvature
}

\author{
RICHARD S. HAMILTON
}

\section{Table of Contents.}

1. Positive Isotropic Curvature $\ldots \ldots \ldots \ldots \ldots \ldots \ldots \ldots \ldots \ldots \ldots$

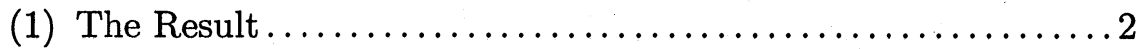

(2) The Algebra of Isotropic Curvature.................

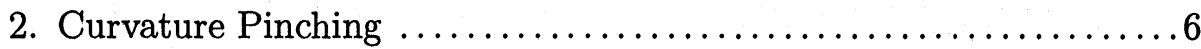

(1) Pinching Estimates which are Preserved $\ldots \ldots \ldots \ldots \ldots \ldots$

(2) Pinching Estimates which Improve ................ 13

(3) Necklike Curvature Pinching $\ldots \ldots \ldots \ldots \ldots \ldots \ldots \ldots \ldots$

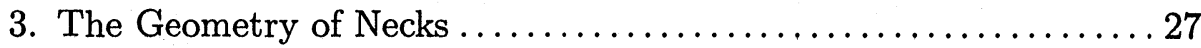

(1) Harmonic Parametrizations by Spheres ..............27

(2) Geometric Necks .............................. 30

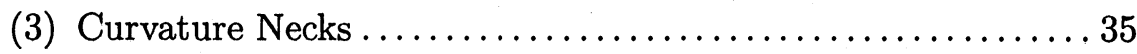

(4) The Fundamental Group .....................41

(5) Finding Necks........................... 44

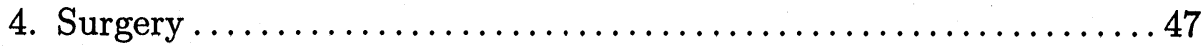

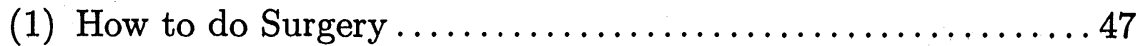

(2) Curvature Changes under Surgery ................49

(3) Pinching Estimates under Surgery ............... 57

5. Recovering the Manifold from Surgery $\ldots \ldots \ldots \ldots \ldots \ldots \ldots \ldots$

\footnotetext{
${ }^{1}$ research supported by NSF grant \# DMS92-04336
} 


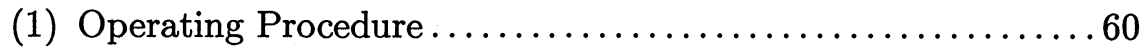

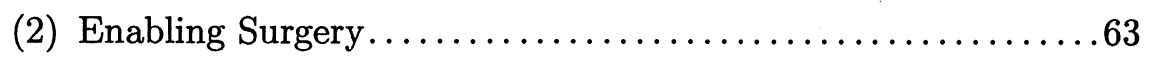

(3) Ancient Solutions with Necklike Ends................. 71

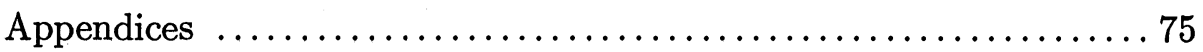

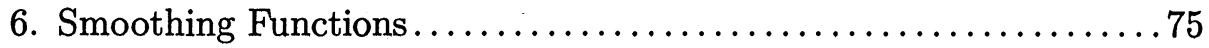

(1) Heat Flow Estimates on Tensors .................. 75

(2) The Smoothing Procedure $\ldots \ldots \ldots \ldots \ldots \ldots \ldots \ldots \ldots \ldots \ldots \ldots \ldots \ldots \ldots$

7. A replacement for Schoenfliess Conjecture $\ldots \ldots \ldots \ldots \ldots 8$

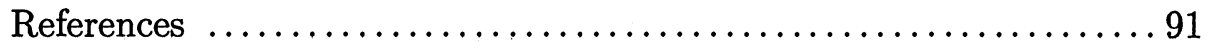

\section{Positive Isotropic Curvature.}

\subsection{The Result.}

A Riemannian manifold of dimension at least 4 is said to have positive isotropic curvature if for every orthonormal 4-frame the curvature tensor satisfies

$$
R_{1313}+R_{1414}+R_{2323}+R_{2424} \geq 2 R_{1234} .
$$

Using minimal surface theory, Micallef and Moore proved [13] that any compact simply connected manifold with positive isotropic curvature is homeomorphic to the sphere $S^{n}$. In this paper we improve on this result in the case where the dimension is exactly four, using the Ricci Flow.

An incompressible space form $N^{3}$ in a four-manifold $M^{4}$ is a smooth submanifold of dimension three diffeomorphic to the quotient $S^{3} / \Gamma$ of the three-sphere by a group of linear isometries without fixed points, such that the fundamental group $\pi_{1}\left(N^{3}\right)$ injects into $\pi_{1}\left(M^{4}\right)$. We say the space form is essential unless $\Gamma=\{1\}$, or $\Gamma=Z_{2}$ and the normal bundle is non-orientable.

Theorem 1.1 Main Theorem. Let $M^{4}$ be a compact four-manifold with no essential incompressible space-form. Then $M^{4}$ admits a metric of positive isotropic curvature if and only if $M^{4}$ is diffeomorphic to the sphere $S^{4}$, the projective space $R P^{4}$, the product $S^{3} \times S^{1}$, the twisted product $S^{3} \widetilde{\times} S^{1}$ which is the only unoriented $S^{3}$ bundle over $S^{1}$, or a connected sum of the above. 
Corollary 1.2. If $M^{4}$ is a compact four-manifold with positive isotropic curvature, then

- (a) if $\pi_{1}=\{1\}, M^{4}$ is diffeomorphic to $S^{4}$

- (b) if $\pi_{1}=Z_{2}, M^{4}$ is diffeomorphic to $R P^{4}$

- (c) if $\pi_{1}=Z, M^{4}$ is diffeomorphic to $S^{3} \times S^{1}$ if it is oriented, and to $S^{3} \widetilde{\times} S^{1}$ if it is not.

In four dimensions it is an open question to date whether there are any exotic differentiable structures on $S^{4}$. Therefore 1.2(a) is possibly an improvement on the result of Micallef and Moore, as their argument only proves that the manifold is a homotopy sphere and then uses Freedman's result. (By contrast, in dimensions 5 and 6 the differentiable structure on $S^{5}$ and $S^{6}$ is unique.) The quarter-pinching hypothesis of Klingenberg is stronger, but Klingenberg writes the manifold as a union of two balls, and in four dimensions such a manifold is known to be diffeomorphic to $S^{4}$. In the case $\pi_{1}\left(M^{4}\right)=Z_{2}$ we certainly get a new result in $1.2(\mathrm{~b})$, as there is known to exist an exotic $Z_{2}$ action on $S^{4}$ such that the quotient is a fake $R P^{4}$, homeomorphic to the standard one but not diffeomorphic. The standard $R P^{4}$ admits a metric of positive isotropic curvature, but the fake one does not. Hence this curvature pinching hypothesis is sensitive to the differentiable structure.

The theorem will be proved by using a surgically modified Ricci flow where we halt the flow at certain times and change the topology. After flowing for a while, we recognize a neck, a region where the metric is very close to the product metric on $S^{3} \times B^{1}$ where $B^{1}$ is an interval, or a quotient of this by a finite group acting freely. When there is no quotient in question, we replace $S^{3} \times B^{1}$ with two copies of the ball $B^{4}$ by cutting the neck and rounding off the ends, in a way we will describe precisely later in terms of what happens to the metric. When the group is $Z_{2}$ acting antipodally on $S^{3}$ and by reflection on $B^{1}$, we can do the quotient surgery to eliminate an $R P^{4}$ summand. For other quotients we get one of the named obstructions $S^{3} / \Gamma$.

Surgery may disconnect the manifold into a number of pieces. If one of these pieces is diffeomorphic to one of the standard models, we throw it away. We can of course recognize such a piece as soon as its curvature operator becomes positive, by [4]. After a finite number of surgeries in a finite time, and discarding a finite number of pieces, we are left with nothing. We can then recover the original manifold by starting with some collection 
of standard spaces and doing surgeries replacing two $B^{4}$ 's with an $S^{3} \times B^{1}$, or one $B^{4}$ with $S^{3} \times B^{1} / Z_{2}$ as described before, which is equivalent to adding an $R P^{4}$ by connected sum anyway. If we do extra surgeries of the manifold to itself this is equivalent to adding $S^{3} \times S^{1}$ 's or $S^{3} \widetilde{\times} S^{1}$ 's by connected sum. This will prove the theorem.

\subsection{The Algebra of Isotropic Curvature.}

We start by explaining positive isotropic curvature. Given a real vector space $V$ we can consider its complexification

$$
\mathbb{C} \otimes_{R} V .
$$

If $V$ has an inner product $\langle$,$\rangle , we can extend it to be complex-linear in$ both arguments (not Hermitian linear). We then say that a complex vector $Z$ is isotropic if $\langle Z, Z\rangle=0$. If $Z=X+i Y$, so that $X$ and $Y$ are its real and imaginary parts, then $Z$ is isotropic if and only if $X \perp Y$ and $|X|=|Y|$. We say that a complex two-plane is isotropic if every vector in it is isotropic. Every complex isotropic two-plane $P$ can be spanned by two vectors $Z=X+i Y$ and $W=U+i V$ where $X, Y . U$, and $V$ are orthonormal. Note there are no complex isotropic two-planes unless the dimension is at least four. We define the sectional curvature of the complex isotropic tangent two-plane $P$ by

$$
K(P)=R(Z, W, \bar{Z}, \bar{W})
$$

for any such basis $Z$ and $W$ for $P$, extending the Riemannian curvature tensor to be complex-linear in all its arguments. Naturally $K(P)$ is real. Using the Bianchi identity we find that

$$
\begin{aligned}
K(P) & =R(X, U, X, U)+R(X, V, X, V)+R(Y, U, Y, U) \\
& +R(Y, V, Y, V)-2 R(X, Y, U, V) .
\end{aligned}
$$

We then say that the metric has positive isotropic curvature if $K(P)>0$ for every complex isotropic tangent two-plane.

In four dimensions it is natural to decompose the real two-forms $\Lambda^{2}$ into the direct sum of $\Lambda_{+}^{2}$ and $\Lambda_{-}^{2}$. Then the curvature operator on two-forms decomposes as a block matrix

$$
M=\left(\begin{array}{cc}
A & B \\
{ }^{t} B & C
\end{array}\right)
$$

as explained in [3]. Consider a positively oriented orthonormal basis $X_{1}, X_{2}, X_{3}, X_{4}$ and let $P$ be the complex isotropic tangent two-plane 
spanned by $X_{1}+i X_{2}$ and $X_{3}+i Y_{4}$. A basis for $\Lambda_{+}^{2}$ (under the obvious duality) is

$\varphi_{1}=X_{1} \wedge X_{2}+X_{3} \wedge X_{4} \quad \varphi_{2}=X_{1} \wedge X_{3}+X_{4} \wedge X_{2} \quad \varphi_{3}=X_{1} \wedge X_{4}+X_{2} \wedge X_{3}$

while a basis for $\Lambda_{-}^{2}$ is

$\psi_{1}=X_{1} \wedge X_{2}-X_{3} \wedge X_{4} \quad \psi_{2}=X_{1} \wedge X_{3}-X_{4} \wedge X_{2} \quad \psi_{3}=X_{1} \wedge X_{4}-X_{2} \wedge X_{3}$.

We can then compute

$$
\begin{aligned}
& A_{11}=R\left(\varphi_{1}, \varphi_{1}\right)=R_{1212}+R_{3434}+2 R_{1234} \\
& A_{22}=R\left(\varphi_{2}, \varphi_{2}\right)=R_{1313}+R_{4242}+2 R_{1342} \\
& A_{33}=R\left(\varphi_{3}, \varphi_{3}\right)=R_{1414}+R_{2323}+2 R_{1423}
\end{aligned}
$$

and

$$
\begin{aligned}
& C_{11}=R\left(\varphi_{1}, \varphi_{1}\right)=R_{1212}+R_{3434}+2 R_{1234} \\
& C_{22}=R\left(\varphi_{2}, \varphi_{2}\right)=R_{1313}+R_{4242}+2 R_{1342} \\
& C_{33}=R\left(\varphi_{3}, \varphi_{3}\right)=R_{1414}+R_{2323}+2 R_{1423} .
\end{aligned}
$$

The sectional curvature of the complex isotropic tangent two-plane $P$ is

$$
K(P)=R_{1313}+R_{1414}+R_{2323}+R_{2424}-2 R_{1234}
$$

and by the Bianchi identity

$$
R_{1234}+R_{1342}+R_{1423}=0 .
$$

Thus we see that

$$
K(P)=A_{22}+A_{33}
$$

for a positively oriented basis. If the basis had the opposite orientation, we would get

$$
K(P)=C_{22}+C_{33} .
$$

Since either can occur, we get the following characterization.

Lemma 2.1. A four-manifold has positive isotropic curvature if and only if

$$
a_{1}+a_{2}>0 \text { and } c_{1}+c_{2}>0
$$

where $a_{1}$ and $a_{2}$ are the two smallest eigenvalues if the matrix $A$, and $c_{1}$ and $c_{2}$ are the two smallest eigenvalues of the matrix $C$. 
For example, the spaces $S^{4}$ and $S^{3} \times R^{1}$ have curvature operators

$$
M_{S^{4}}=\left(\begin{array}{cc}
I & 0 \\
0 & I
\end{array}\right) \quad M_{S^{3} \times R^{1}}=\left(\begin{array}{cc}
I & I \\
I & I
\end{array}\right)
$$

and hence clearly have strictly positive isotropic curvature, while $S^{2} \times S^{2}$ and $S^{2} \times R^{2}$ and $\mathbb{C} P^{2}$ have curvature operators

$$
\begin{gathered}
M_{S^{2} \times S^{2}}=\left(\begin{array}{cc}
E & 0 \\
0 & E
\end{array}\right) \quad M_{S^{2} \times R^{2}}=\left(\begin{array}{ll}
E & E \\
E & E
\end{array}\right) \\
M_{\mathbb{C} P^{2}}=\left(\begin{array}{cc}
I & 0 \\
0 & 3 E
\end{array}\right)
\end{gathered}
$$

where $E$ is the matrix

$$
E=\left(\begin{array}{lll}
1 & 0 & 0 \\
0 & 0 & 0 \\
0 & 0 & 0
\end{array}\right),
$$

and thus these spaces only have non-negative curvature operator.

The trace of the $A$ matrix and the trace of the $C$ matrix are equal by the Bianchi identity, and both equal scalar curvature, up to a factor. The tracefree part of $A$ is the self-dual part $W_{+}$of the Weyl tensor, and the trace-free part of $C$ is the anti-self-dual part $W_{-}$. The matrix $B$ gives the traceless Ricci tensor. If a manifold is conformally flat with positive scalar curvature, then $A$ and $C$ are the same positive multiple of the identity matrix, and hence the manifold has positive isotropic curvature. The connected sum of two conformally flat manifolds can be made conformally flat, and the connected sum of two manifolds of positive scalar curvature can be made to have positive scalar curvature. Moreover these constructions are compatible. Hence a connected sum of $R P^{4}$ 's and $S^{3} \times S^{1}$ 's and $\left(S^{3} / \Gamma\right) \times S^{1}$ 's (or other quotients if $S^{3} \times S^{1}$ ) has a metric which is conformally flat with positive scalar curvature, and hence has positive isotropic curvature. This is the converse of our result. We leave the details to the reader.

\section{Curvature Pinching.}

\subsection{Pinching Estimates which are Preserved.}

In this section and the next we prove the following result. 
Theorem 1.1 Pinching Theorem. Given an initial metric on a compact four-manifold with strictly positive isotropic curvature, there exists $\rho>0$ and $\Omega<\infty$ depending only on the initial metric, such that for every $\delta>0$ there exists a constant $K$ depending only on $\rho, \Omega, \delta$ and the initial metric, with the following property. The subsequent solution to the Ricci Flow has

$$
a_{1}+\rho>0 \text { and } c_{1}+\rho>0
$$

and

$$
\max \left(a_{3}, b_{3}, c_{3}\right) \leq \Omega\left(a_{1}+\rho\right) \quad \text { and } \quad \max \left(a_{3}, b_{3}, c_{3}\right) \leq \Omega\left(c_{1}+\rho\right)
$$

and

$$
b_{3} \leq(1+\delta) \sqrt{\left(a_{1}+\rho\right)\left(c_{1}+\rho\right)}+K
$$

at all points and all times.

This result will be a Corollary of the last of a sequence of estimates, each of which is preserved by the Ricci Flow.

We follow the same procedure as in [3] to reduce the proof to the preservation of inequalities defining convex sets when we evolve the curvature matrix by a system of ordinary differential inequalities. We assume the reader is familiar with this paper.

Theorem 1.2. The Ricci flow on a compact 4-manifold preserves positive isotropic curvature. For any constant $m \geq 0$ the Ricci flow preserves the inequalities

$$
a_{1}+a_{2} \geq m \quad \text { and } \quad c_{1}+c_{2} \geq m
$$

Proof. From the ordinary differential inequalities in [3]

$$
\frac{d}{d t}\left(a_{1}+a_{2}\right) \geq a_{1}^{2}+a_{2}^{2}+2\left(a_{1}+a_{2}\right) a_{3}+b_{1}^{2}+b_{2}^{2} .
$$

Now if $a_{1}+a_{2} \geq m \geq 0$ then $a_{3} \geq 0$ also. The set $a_{1}+a_{2} \geq m$ is convex since $a_{1}+a_{2}$ is a concave function of the matrix $A$. This proves the theorem for $A$, and $C$ is the same. From now on we always assume $a_{1}+a_{2}>0$ and $c_{1}+c_{2}>0$.

Theorem 1.3. The Ricci flow preserves the inequality

$$
\left(b_{2}+b_{3}\right)^{2} \leq \Lambda\left(a_{1}+a_{2}\right)\left(c_{1}+c_{2}\right)
$$

for any constant $\Lambda \geq 0$. 
Proof. We have

$$
\begin{aligned}
& \frac{d}{d t} \ln \left(a_{1}+a_{2}\right) \geq 2 a_{3}+2 b_{1}+\frac{\left(a_{1}-b_{1}\right)^{2}+\left(a_{2}-b_{2}\right)^{2}+2 a_{2}\left(b_{2}-b_{1}\right)}{a_{1}+a_{2}} \\
& \frac{d}{d t} \ln \left(b_{2}+b_{3}\right) \leq a_{3}+2 b_{1}+c_{3}-\frac{\left[\left(a_{3}-a_{2}\right)+\left(c_{3}-c_{2}\right)\right] b_{2}}{b_{2}+b_{3}} \\
& \frac{d}{d t} \ln \left(c_{1}+c_{2}\right) \geq 2 b_{1}+2 c_{3}+\frac{\left(c_{1}-b_{1}\right)^{2}+\left(c_{2}-b_{2}\right)^{2}+2 c_{2}\left(b_{2}-b_{1}\right)}{c_{1}+c_{2}}
\end{aligned}
$$

and it follows then

$$
\frac{d}{d t} \ln \frac{\left(b_{2}+b_{3}\right)^{2}}{\left(a_{1}+a_{2}\right)\left(c_{1}+c_{2}\right)} \leq 0
$$

which shows the set is preserved. Now $a_{1}+a_{2}$ and $c_{1}+c_{2}$ concave functions, while $b_{2}+b_{3}$ is convex. Moreover the set

$$
y^{2} \leq \Lambda x z
$$

is convex for any $\Lambda$. Therefore the inequality defines a convex set if matrices, and we are done. Note that if $M^{4}$ is compact and $a_{1}+a_{2}>0$ and $c_{1}+c_{2}>0$ at $t=0$, there is some constant $\Lambda$ so that the estimate indeed holds at $t=0$, and now for $t \geq 0$.

Theorem 1.4. If the previous estimate

$$
\left(b_{2}+b_{3}\right)^{2} \leq \Lambda\left(a_{1}+a_{2}\right)\left(c_{1}+c_{2}\right)
$$

holds, and if $\Phi$ is any constant with

$$
\Phi \geq \Lambda+1
$$

then the estimates

$$
a_{2}+a_{3} \leq \Phi\left(a_{1}+a_{2}\right) \quad \text { and } \quad c_{2}+c_{3} \leq \Phi\left(c_{1}+c_{2}\right)
$$

are also preserved by the Ricci flow.

Proof. We have

$$
\frac{d}{d t}\left(a_{2}+a_{3}\right) \leq a_{2}^{2}+a_{3}^{2}+2 a_{1}\left(a_{2}+a_{3}\right)+b_{1}^{2}+b_{2}^{2} .
$$

Now

$$
a_{2}^{2}+a_{3}^{2} \leq a_{3}\left(a_{2}+a_{3}\right)
$$


and

$$
b_{1}^{2}+b_{2}^{2} \leq\left(b_{1}+b_{2}\right)^{2} \leq \Lambda\left(a_{1}+a_{2}\right)\left(c_{1}+c_{2}\right)
$$

and

$$
c_{1}+c_{2} \leq \frac{2}{3}\left(c_{1}+c_{2}+c_{3}\right)=\frac{2}{3}\left(a_{1}+a_{2}+a_{3}\right) \leq a_{2}+a_{3}
$$

since $a_{1}+a_{2}+a_{3}=c_{1}+c_{2}+c_{3}$ is the first Bianchi identity for $n=4$. Thus

$$
\frac{d}{d t} \ln \left(a_{2}+a_{3}\right) \leq a_{3}+2 a_{1}+\Lambda\left(a_{1}+a_{2}\right) .
$$

Also we have

$$
\frac{d}{d t}\left(a_{1}+a_{2}\right) \geq a_{1}^{2}+a_{2}^{2}+2\left(a_{1}+a_{2}\right) a_{3}+b_{1}^{2}+b_{2}^{2}
$$

and

$$
a_{1}^{2}+a_{2}^{2} \geq a_{1}\left(a_{1}+a_{2}\right)
$$

which easily gives

$$
\frac{d}{d t} \ln \left(a_{1}+a_{2}\right) \geq a_{1}+2 a_{3} .
$$

To preserve $a_{2}+a_{3} \leq \Phi\left(a_{1}+a_{2}\right)$ we need

$$
a_{3}+2 a_{1}+\Lambda\left(a_{1}+a_{2}\right) \leq a_{1}+2 a_{3}
$$

which is the same as

$$
(\Lambda+1)\left(a_{1}+a_{2}\right) \leq a_{2}+a_{3} .
$$

Now on the boundary of the inequality

$$
a_{2}+a_{3}=\Phi\left(a_{1}+a_{2}\right)
$$

and if $\Phi \geq \Lambda+1$ we are done. The other estimate is the same.

Corollary 1.5. If the above estimates hold, we can find a constant $\Sigma=$ $\Phi+1$ such that

$$
\max \left(a_{3}, b_{3}, c_{3}\right) \leq \Sigma\left(a_{1}+a_{2}\right) \quad \text { and } \max \left(a_{3}, b_{3}, c_{3}\right) \leq \Sigma\left(c_{1}+c_{2}\right)
$$

and

$$
R \leq \Sigma\left(a_{1}+a_{2}\right) \text { and } R \leq \Sigma\left(c_{1}+c_{2}\right) \text {. }
$$


Proof. Since $a_{2} \geq 0$ we use

$$
a_{3} \leq a_{2}+a_{3} \leq \Phi\left(a_{1}+a_{2}\right)
$$

Since $c_{1}+c_{2} \geq 0$ we use

$c_{3} \leq c_{1}+c_{2}+c_{3}=a_{1}+a_{2}+a_{3} \leq\left(a_{1}+a_{2}\right)+\left(a_{2}+a_{3}\right) \leq(\Phi+1)\left(a_{1}+a_{2}\right)$.

We have already used $c_{1}+c_{2} \leq a_{2}+a_{3}$, so

$$
\left(b_{2}+b_{3}\right)^{2} \leq \Lambda\left(a_{1}+a_{2}\right)\left(c_{1}+c_{2}\right) \leq \Lambda \Phi\left(a_{1}+a_{2}\right)^{2}
$$

and we use $b_{2} \geq 0$ to get

$$
b_{3} \leq b_{2}+b_{3} \leq \sqrt{\Lambda \Phi}\left(a_{1}+a_{2}\right) .
$$

Since $\Phi \geq \Lambda+1 \geq \Lambda$ we can take $\Sigma=\Phi+1$. Also

$$
R=a_{1}+a_{2}+a_{3}=c_{1}+c_{2}+c_{3}
$$

so

$$
R \leq\left(a_{1}+a_{2}\right)+\left(a_{2}+a_{3}\right)
$$

and

$$
R \leq \Sigma\left(a_{1}+a_{2}\right)
$$

as desired.

Theorem 1.6. For any constant $\rho$ the estimates

$$
a_{1}+\rho \geq 0 \quad \text { and } \quad c_{1}+\rho \geq 0
$$

are preserved by the Ricci flow.

Proof. We use

$$
\frac{d}{d t} a_{1} \geq a_{1}^{2}+2 a_{2} a_{3}+b_{1}^{2}
$$

and if $a_{1}+a_{2} \geq 0$ then $a_{2} \geq 0$ and $a_{3} \geq 0$ so $a_{2} a_{3} \geq 0$.

Theorem 1.7. If the previous estimates

$$
\max \left(a_{3}, b_{3}, c_{3}\right) \leq \Sigma\left(a_{1}+a_{2}\right) \quad \text { and } \quad \max \left(a_{3}, b_{3}, c_{3}\right) \leq \Sigma\left(c_{1}+c_{2}\right)
$$


hold and $a_{1}+\rho \geq 0$ and $c_{1}+\rho \geq 0$ and if $\Psi$ is any constant with

$$
\Psi \geq 4 \Sigma^{2}+1
$$

then the estimates

$$
a_{3} \leq \Psi\left(a_{1}+\rho\right) \quad \text { and } \quad c_{3} \leq \Psi\left(c_{1}+\rho\right)
$$

are preserved by the Ricci flow.

Proof. Since $a_{1}+a_{2} \leq 2 a_{2}$ we have

$$
\max \left(a_{3}, b_{3}, c_{3}\right) \leq 2 \Sigma a_{2} .
$$

Now $a_{3}^{2} \leq 4 \Sigma^{2} a_{2}^{2}$ and $b_{3}^{2} \leq 4 \Sigma^{2} a_{2}^{2}$ and $2 a_{1} a_{2} \leq 2 a_{2}^{2}$ and

$$
\frac{d}{d t} a_{3} \leq a_{3}^{2}+2 a_{1} a_{2}+b_{3}^{2} \leq\left(8 \Sigma^{2}+2\right) a_{2}^{2}
$$

and hence using $a_{2} \leq a_{3}$

$$
\frac{d}{d t} \ln a_{3} \leq\left(8 \Sigma^{2}+2\right) a_{2} .
$$

On the other hand

$$
\frac{d}{d t}\left(a_{1}+\rho\right) \geq a_{1}^{2}+2 a_{2} a_{3}+b_{1}^{2} \geq 2 a_{2} a_{3}
$$

and on the boundary of the inequality we have

$$
a_{3}=\Psi\left(a_{1}+\rho\right) .
$$

This gives

$$
\frac{d}{d t} \ln \left(a_{1}+\rho\right) \geq 2 \Psi a_{2} .
$$

Then the inequality is preserved if

$$
2 \Psi \geq 8 \Sigma^{2}+2
$$

which reduces to our hypothesis. Since $a_{3}$ is convex and $a_{1}+\rho$ is concave, the inequality defines a convex set. This proves the theorem. The other estimate is the same. 
Corollary 1.8. If the above estimates hold, we can find a constant $\Omega=\Sigma \Psi$ such that

$$
\max \left(a_{3}, b_{3}, c_{3}\right) \leq \Omega\left(a_{1}+\rho\right) \text { and } \max \left(a_{3}, b_{3}, c_{3}\right) \leq \Omega\left(c_{1}+\rho\right)
$$

Proof. We have

$$
\max \left(a_{3}, b_{3}, c_{3}\right) \leq \Sigma\left(a_{1}+a_{2}\right)
$$

and $a_{2} \leq a_{3} \leq \Psi\left(a_{1}+\rho\right)$ so the estimate holds with $\Omega=\Sigma \Psi$. The other is the same.

Theorem 1.9. If the previous estimates

$$
\begin{aligned}
& a_{1}+\rho>0 \text { and } \\
& c_{1}+\rho>0 \\
& \max \left(a_{3}, b_{3}, c_{3}\right) \leq \Omega\left(a_{1}+\rho\right) \text { and } \max \left(a_{3}, b_{3}, c_{3}\right) \leq \Omega\left(c_{1}+\rho\right)
\end{aligned}
$$

hold, then for any constant $\Xi \geq 0$ the inequality

$$
b_{3} \leq \Xi e^{P t} \sqrt{\left(a_{1}+\rho\right)\left(c_{1}+\rho\right)}
$$

is preserved by the Ricci flow with $P=4 \Omega \rho$.

Proof. We have

$$
\frac{d}{d t} b_{3} \leq\left(a_{3}+c_{3}\right) b_{3}+2 b_{1} b_{2}
$$

and this makes

$$
\frac{d}{d t} \ln b_{3} \leq a_{3}+b_{3}+2 b_{1}
$$

On the other hand

$$
\frac{d}{d t}\left(a_{1}+\rho\right) \geq a_{1}^{2}+2 a_{2} a_{3}+b_{1}^{2}
$$

and this makes

$$
\frac{d}{d t} \ln \left(a_{1}+\rho\right) \geq 2 a_{3}+2 b_{1}-\frac{2\left(a_{3}+b_{1}\right) \rho}{a_{1}+\rho}
$$

Now we use our hypothesis to bound

$$
2\left(a_{3}+b_{1}\right) \leq 4 \Omega\left(a_{1}+\rho\right)
$$


and we get

$$
\frac{d}{d t} \ln \left(a_{1}+\rho\right) \geq 2 a_{3}+2 b_{1}-4 \Omega \rho
$$

Likewise

$$
\frac{d}{d t} \ln \left(c_{1}+\rho\right) \geq 2 c_{3}+2 b_{1}-4 \Omega \rho
$$

and we have

$$
\frac{d}{d t} \ln \frac{b_{3}}{\sqrt{\left(a_{1}+\rho\right)\left(c_{1}+\rho\right)}} \leq 4 \Omega \rho=P
$$

which shows the inequality is preserved by the Ricci Flow. Since $y^{2} \leq C x z$ is a convex set, this proves the Theorem.

We remark that $\Omega$ and $\rho$ can be chosen in term of the data at $t=0$, and the minimum of the scalar curvature at $t=0$ determines an upper bound $T$ on the time the solution can exist. This gives a uniform bound on the constant $e^{8 \Omega \rho t}$.

\subsection{Pinching Estimates which Improve.}

In this part we show that as the curvature gets big the isotropic pinching improves. A similar but easier result holds in dimension 3 without curvature restrictions (see [10]).

Theorem 2.1. For any constants $\Lambda$ and $\Omega$ as before we can find a constant $K$ such that the inequality

$$
\frac{2 b_{3}}{\sqrt{\left(a_{1}+a_{2}\right)\left(c_{1}+c_{2}\right)}} \leq 1+\frac{K}{\max \left\{\ln \sqrt{\left(a_{1}+a_{2}\right)\left(c_{1}+c_{2}\right)}, 2\right\}}
$$

is preserved by the Ricci Flow.

Proof. Again we must show the inequality defines a convex set of matrices preserved by the ordinary differential inequalities. To see the set is convex, observe that $a_{1}+a_{2}$ and $c_{1}+c_{2}$ are concave, so

$$
x=\sqrt{\left(a_{1}+a_{2}\right)\left(c_{1}+c_{2}\right)}
$$

is concave, while

$$
y=2 b_{3}
$$


is convex. Then if $y=f(x)$ is a concave increasing function, the set $y \leq$ $f(x)$ will be convex set, because a concave increasing function of a concave function is concave. We can write our set in this form where

$$
y= \begin{cases}\left(1+\frac{K}{\ln x}\right) x & \text { for } x \geq e^{2} \\ \left(1+\frac{K}{2}\right) x & \text { for } x \leq e^{2} .\end{cases}
$$

Note both definitions agree at $x=e^{2}$. Also $y$ is increasing since $y^{\prime} \geq 1$; indeed

$$
y^{\prime}= \begin{cases}1+\frac{K}{\ln x}-\frac{K}{\ln ^{2} x} & \text { for } x \geq e^{2} \\ 1+\frac{K}{2} & \text { for } x \leq e^{2}\end{cases}
$$

and since at $x=e^{2}$ the derivative from the left is greater than the derivative from the right, there is no problem with concavity there. Then

$$
y^{\prime \prime}=\left\{\begin{array}{cc}
-\frac{K(\ln x-2)}{x \ln ^{3} x} & \text { for } x>e^{2} \\
0 & \text { for } x<e^{2} .
\end{array}\right.
$$

and in either case we have $y^{\prime \prime} \leq 0$. This shows our set of matrices is convex.

Now we must check the differential inequalities. When

$$
x=\sqrt{\left(a_{1}+a_{2}\right)\left(c_{1}+c_{2}\right)} \leq e^{2}
$$

we can take $K$ so large that

$$
1+\frac{K}{2} \geq \Lambda
$$

and then the inequality

$$
\frac{y}{x} \leq 1+\frac{K}{2}
$$

is preserved by our previous pinching estimate. So we only need to check when $x \geq e^{2}$. Then what we need to show is that

$$
\frac{d y}{d t} \leq f^{\prime}(x) \frac{d x}{d t}
$$

when $y=f(x)$ and $x \geq e^{2}$, since we can only leave the designated region at the boundary. Recall from [3] that the inequality is understood in the sense of lim sup (on the left) and lim inf (on the right) of forward difference quotients, since $x$ and $y$ may not be differentiable everywhere but only Lipschitz continuous (being the eigenvalues of a matrix). 
By the differential inequalities

$$
\begin{aligned}
& \frac{d}{d t} \ln \left(a_{1}+a_{2}\right) \geq 2 a_{3}+2 b_{1}+\frac{\left(a_{1}-b_{1}\right)^{2}+\left(a_{2}-b_{2}\right)^{2}+2 a_{2}\left(b_{2}-b_{1}\right)}{a_{1}+a_{2}} \\
& \frac{d}{d t} \ln \left(c_{1}+c_{2}\right) \geq 2 c_{3}+2 b_{1}+\frac{\left(c_{1}-b_{1}\right)^{2}+\left(c_{2}-b_{2}\right)^{2}+2 c_{2}\left(b_{2}-b_{1}\right)}{c_{1}+c_{2}}
\end{aligned}
$$

and

$$
\frac{d}{d t} \ln b_{3} \leq a_{3}+c_{3}+2 b_{1}-\frac{2 b_{1}\left(b_{3}-b_{2}\right)}{b_{3}} .
$$

From this we see that

$$
\frac{d}{d t} \ln x \geq D+E_{1} \quad \text { and } \quad \frac{d}{d t} \ln y \leq D+E_{2}
$$

where

$$
D=a_{3}+c_{3}+2 b_{1}
$$

and

$E_{1}=\frac{\left(a_{1}-b_{1}\right)^{2}+\left(a_{2}-b_{2}\right)^{2}+2 a_{2}\left(b_{2}-b_{1}\right)}{2\left(a_{1}+a_{2}\right)}+\frac{\left(c_{1}-b_{1}\right)^{2}+\left(c_{2}-b_{2}\right)^{2}+2 c_{2}\left(b_{2}-b_{1}\right)}{2\left(c_{1}+c_{2}\right)}$

and

$$
E_{2}=\frac{2 b_{1}\left(b_{3}-b_{2}\right)}{b_{3}}
$$

We need to show that

$$
D+E_{2} \leq\left[1+\frac{K}{\ln x}-\frac{K}{\ln ^{2} x}\right]\left(D+E_{1}\right)
$$

when

$$
y=\left(1+\frac{K}{\ln x}\right) x
$$

Solve the latter to get

$$
\ln x=\frac{K x}{y-x}
$$

and substitute this above. If we simplify the resulting inequality with $\lambda=$ $y / x$, it suffices to show

$$
(\lambda-1)^{2}\left(D+E_{2}\right) \leq K \lambda\left(E_{1}+E_{2}\right) .
$$

Now for $x \geq e^{2}$ surely $\lambda \geq 1$, so we can get by with

$$
(\lambda-1)^{2}\left(D+E_{2}\right) \leq K\left(E_{1}+E_{2}\right) .
$$


Moreover $\lambda \leq \Lambda$ always, so if $K$ is large compared to $\Lambda$ we get

$$
(\Lambda-1)^{2} \leq \frac{1}{2} K
$$

and then it suffices to have

$$
(\lambda-1)^{2} D \leq \frac{1}{2} K\left(E_{1}+E_{2}\right)
$$

Since $D \leq C|R m|$ for some $C$, it suffices to take $K \geq 2 C / \delta$ where $\delta$ is given by the following estimate.

Lemma 2.2. There exists $a \delta>0$ depending on $\Lambda$ and $\Omega$ such that

$$
E_{1}+E_{2} \geq \delta(\lambda-1)^{2}|R m|
$$

Proof. Clearly

$E_{1} \geq \eta\left\{\left(a_{1}-b_{1}\right)^{2}+\left(a_{2}-b_{2}\right)^{2}+\left(b_{2}-b_{1}\right)^{2}+\left(c_{1}-b_{1}\right)^{2}+\left(c_{2}-b_{2}\right)^{2}\right\} /|R m|$

for some constant $\eta>0$. Now $a_{1}+a_{2}$ is at least a fraction of $|R m|$, so unless $a_{1}, a_{2}, b_{1}, b_{2}, c_{1}, c_{2}$ are all at least a fraction of $|R m|$, we would have $E_{1}$ a fraction of $|R m|$, and we could find $\delta$ as desired since $\lambda \leq \Lambda$ and $E_{2} \geq 0$. Since $b_{1}$ is a fraction of $|R m|$ and $b_{3}$ is bounded by $|R m|$, we get

$$
E_{2} \geq \eta\left(b_{3}-b_{2}\right)^{2} /|R m|
$$

for some constant $\eta>0$.

Now we use

$$
\lambda=\frac{2 b_{3}}{\sqrt{\left(a_{1}+a_{2}\right)\left(c_{1}+c_{2}\right)}} \leq \frac{2 b_{3}}{\min \left\{a_{1}+a_{2}, c_{1}+c_{2}\right\}}
$$

to see

$$
\lambda-1 \leq \max \left\{\frac{2 b_{3}}{a_{1}+a_{2}}-1, \frac{2 b_{3}}{c_{1}+c_{2}}-1\right\} .
$$

When $|R m| \leq \Omega\left(a_{1}+a_{2}\right)$ and $|R m| \leq \Omega\left(c_{1}+c_{2}\right)$, this makes

$$
\lambda-1 \leq C\left(\left|b_{3}-a_{1}\right|+\left|b_{3}-a_{2}\right|+\left|b_{3}-c_{1}\right|+\left|b_{3}-c_{2}\right|\right) /|R m|
$$

for some constant depending on $\Omega$. Then

$$
(\lambda-1)^{2}|R m| \leq C\left(E_{1}+E_{2}\right)
$$

for some $C$ depending on $\Lambda$ and $\Omega$, and we take $\delta=1 / C$. 
Theorem 2.3. We can find constants $L, P$, and $Q$ depending on $\Lambda, \Omega, \rho$ and $K$ given as before, much that the inequality

$$
\frac{b_{3}}{\sqrt{\left(a_{1}+\rho\right)\left(c_{1}+\rho\right)}} \leq 1+\frac{L e^{P t}}{\max \left\{\ln \sqrt{\left(a_{1}+\rho\right)\left(c_{1}+\rho\right)}, Q\right\}}
$$

is true at $t=0$ and is preserved by the Ricci flow.

Proof. Clearly if $L$ is large compared to $Q$ and $\Omega$ and the initial maximum of the curvature, then it is true at $t=0$. The inequality defines a convex set for the same reason as before if $Q \geq 2$. Hence it suffices to show the set is preserved by the ordinary differential inequalities. Let

$$
u=\sqrt{\left(a_{1}+\rho\right)\left(c_{1}+\rho\right)} .
$$

For $u \leq e^{Q}$, the set is the same sort preserved before. So we only have to check $u \geq e^{Q}$, where the $\max$ is the logarithm. Let $\mu$ be the ratio

$$
\mu=\frac{b_{3}}{\sqrt{\left(a_{1}+\rho\right)\left(c_{1}+\rho\right)}} .
$$

We are done when we can compare $\mu$ favorably to our previous ratio $\lambda$ and use the bound for $\lambda$ given before. To see this, consider the case where

$$
\frac{a_{2}-a_{1}}{a_{1}+\rho}+\frac{c_{2}-c_{1}}{c_{1}+\rho} \leq 2 \delta(\mu-1)
$$

for some $\delta>0$ we choose later. Then

$$
\frac{a_{1}+a_{2}}{a_{1}+\rho} \leq 2[1+\delta(\mu-1)] \text { and } \frac{c_{1}+c_{2}}{c_{1}+\rho} \leq 2[1+\delta(\mu-1)]
$$

which makes

$$
\mu \leq \lambda[1+\delta(\mu-1)]
$$

which makes

$$
\mu-1 \leq \frac{\lambda-1}{1-\delta \lambda} .
$$

Since $\lambda$ is bounded above in terms of $K$, if we take $\delta$ small compared to $K$ we have $\delta \lambda \leq 1 / 2$ and

$$
\mu-1 \leq 2(\lambda-1) .
$$

But

$$
\lambda-1 \leq \frac{K}{\ln x}
$$


by our previous estimate. Also $a_{1}+\rho$ and $c_{1}+\rho$ are at least a fraction of $|R m|$ while $a_{1}+a_{2}$ and $c_{1}+c_{2}$ are at most a multiple. This makes $u \leq C x$ for some $C$, or

$$
\ln u \leq \ln x+\ln C
$$

and now if $u \geq e^{Q}$ with $Q$ big we easily get

$$
\ln u \leq 2 \ln x
$$

which makes

$$
\mu-1 \leq \frac{4 K}{\ln u}
$$

and we are done in this case with $L \geq 4 K$.

The other case is when

$$
\frac{a_{2}-a_{1}}{a_{1}+\rho}+\frac{c_{2}-c_{1}}{c_{1}+\rho} \geq 2 \delta(\mu-1)
$$

which we assume now. From the differential inequalities

$$
\frac{d}{d t} \ln \left(a_{1}+\rho\right) \geq 2 a_{3}+2 b_{1}+\frac{\left(a_{1}-b_{1}\right)^{2}+2\left(a_{2}-a_{1}\right) a_{3}}{a_{1}+\rho}-\frac{\rho}{a_{1}+\rho}\left(2 a_{3}+2 b_{1}\right) .
$$

Since $a_{3}$ is at least a fraction of $u$ and $a_{1}+\rho$ is at least a fraction of $2 a_{3}+2 b_{1}$, we get

$$
\frac{d}{d t} \ln \left(a_{1}+\rho\right) \geq 2 a_{3}+2 b_{1}+\theta\left(\frac{a_{2}-a_{1}}{a_{1}+\rho}\right) u-C \rho
$$

for some constants $\theta>0$ and $C$; and likewise

$$
\frac{d}{d t} \ln \left(c_{1}+\rho\right) \geq 2 c_{3}+2 b_{1}+\theta\left(\frac{c_{2}-c_{1}}{c_{1}+\rho}\right) u-C \rho .
$$

With our previous estimate we get

$$
\frac{d}{d t} \ln u \geq a_{3}+c_{3}+2 b_{1}+2 \zeta(\mu-1) u-C \rho
$$

for some $\zeta=\theta \delta>0$.

On the boundary of the set we are trying to preserve, the inequality defining the set becomes an equality; so on the boundary

$$
\mu-1=\frac{L e^{P t}}{\ln u} \geq \frac{L}{\ln u}
$$


and so if we take $L$ or $Q$ large enough

$$
\zeta(\mu-1) u \geq \frac{\zeta L u}{\ln u} \geq \frac{\zeta L Q}{\ln Q} \geq C \rho
$$

and this leaves

$$
2 \zeta(\mu-1) u-C \rho \geq \zeta(\mu-1) u .
$$

This gives the cleaner result

$$
\frac{d}{d t} \ln u \geq a_{3}+c_{3}+2 b_{1}+\zeta(\mu-1) u .
$$

On the other hand, we have seen

$$
\frac{d}{d t} \ln b_{3} \leq a_{3}+c_{3}+2 b_{1} .
$$

To keep the notation like before, let $z=b_{3}$, and write

$$
\begin{gathered}
D=a_{3}+c_{3}+2 b_{1} \\
E=\zeta(\mu-1) u .
\end{gathered}
$$

Then we have $\mu=z / u$ and

$$
\frac{d}{d t} \ln u \geq D+E \quad \text { and } \quad \frac{d}{d t} \ln z \leq D
$$

on the boundary of the set where

$$
z \leq\left(1+\frac{L e^{P t}}{\ln u}\right) u
$$

We need to check that this set is preserved by the differential inequalities. Thus we need to show that

$$
\frac{d z}{d t} \leq\left(1+\frac{L e^{P t}}{\ln u}-\frac{L e^{P t}}{\ln ^{2} u}\right) \frac{d u}{d t}+\frac{P L e^{P t}}{\ln u}
$$

on the set where

$$
z=\left(1+\frac{L e^{P t}}{\ln u}\right) u
$$

Solve the latter for

$$
\ln u=\frac{L e^{P t} u}{z-u}
$$


and substitute above (and throw away the final term $P L e^{P t} / \ln u$ ). Then it suffices to show (using our bounds on $d z / d t$ and $d u / d t$ ) that

$$
D z \leq\left[1+\frac{z-u}{u}-\frac{1}{L e^{P t}}\left(\frac{z-u}{u}\right)^{2}\right](D+E) u
$$

which simplifies to

$$
(\mu-1)^{2}(D+E) \leq \mu L e^{P t} E .
$$

Since $\mu-1 \leq \mu$, it suffices to show

$$
(\mu-1)(D+E) \leq L e^{P t} E .
$$

Now in a previous bound in Theorem 1.9 we have

$$
\mu=\frac{b_{3}}{\sqrt{\left(a_{1}+\rho\right)\left(c_{1}+\rho\right)}} \leq \frac{1}{2} L e^{P t}
$$

preserved by the Ricci Flow for any $L=2 \Xi$ and a suitaably large $P=4 \Omega \rho$, and we take our $L$ and $P$ here at least this large. Then

$$
(\mu-1) E \leq \frac{1}{2} L e^{P t} E
$$

and it suffices to show

$$
(\mu-1) D \leq L e^{P t} E
$$

Now we use $e^{P t} \geq 1$ and

$$
D \leq C u \quad \text { and } \quad E=\zeta(\mu-1) u
$$

and it suffices to have $L$ big. This proves the Theorem.

Now it is easy to derive the Pinching Theorem 1.1 as a Corollary. We have already remarked the minimum of the scalar curvature gives an upper bound $T$ on the time the solution exists. Choose $\rho$ as before, let

$$
B=1+\frac{L e^{P T}}{Q}
$$

and given $\delta>0$, choose the constant $K$ in the Theorem as

$$
K=B e^{L e^{P t / \delta}}
$$

If $b_{3} \leq K$ we surely have

$$
b_{3} \leq(1+\delta) \sqrt{\left(a_{1}+\rho\right)\left(c_{1}+\rho\right)}+K
$$


and we are done. Otherwise we always have

$$
b_{3} \leq B \sqrt{\left(a_{1}+\rho\right)\left(c_{1}+\rho\right)}
$$

by the previous estimate using the worst cases where $t=T$ and

$$
\sqrt{\left(a_{1}+\rho\right)\left(c_{1}+\rho\right)} \leq Q .
$$

The bounds on $b_{3}$ give

$$
\sqrt{\left(a_{1}+\rho\right)\left(c_{1}+\rho\right)} \geq K / B
$$

and this makes

$$
\frac{b_{3}}{\sqrt{\left(a_{1}+\rho\right)\left(c_{1}+\rho\right)}} \leq 1+\frac{L e^{P t}}{\ln (K / B)} \leq 1+\delta
$$

and we are done.

\subsection{Necklike Curvature Pinching.}

In this part we prove the following alternative: for a solution with positive isotropic curvature, either there is some point where the curvature is not small compared to the time to blow up and where the curvature tensor is nearly that on a cylinder, or else the curvature operator becomes positive everywhere. We assume our solution has isotropic eccentricities bounded by $\Lambda$ and $\Sigma$ as before, so that

$$
\left(b_{2}+b_{3}\right)^{2} \leq \Lambda\left(a_{1}+a_{2}\right)\left(c_{1}+c_{2}\right)
$$

and

$$
R \leq \Sigma\left(a_{1}+a_{2}\right) \text { and } R \leq \Sigma\left(c_{1}+c_{2}\right) .
$$

By the argument in [3] we know

$$
\begin{gathered}
\frac{d}{d t}\left(a_{1}+a_{2}\right) \geq a_{1}^{2}+2 a_{2} a_{3}+a_{2}^{2}+2 a_{1} a_{3}+b_{1}^{2}+b_{2}^{2} \\
\frac{d}{d t}\left(c_{1}+c_{2}\right) \geq c_{1}^{2}+2 c_{2} c_{3}+c_{2}^{2}+2 c_{1} c_{3}+b_{1}^{2}+b_{2}^{2} \\
\frac{d}{d t} b_{3} \leq a_{3} b_{3}+b_{3} c_{3}+2 b_{1} b_{2} \\
\frac{d}{d t}\left(b_{2}+b_{3}\right) \leq a_{2} b_{2}+b_{2} c_{2}+2 b_{1} b_{3}+a_{3} b_{3}+b_{3} c_{3}+2 b_{1} b_{2}
\end{gathered}
$$

and hence by some algebraic manipulation we get the following result. 
Lemma 3.1. We have

$$
\begin{aligned}
\frac{d}{d t} \ln \frac{\left(b_{2}+2 b_{3}\right)^{2}}{\left(a_{1}+a_{2}\right)\left(c_{1}+c_{2}\right)} \leq & -\left\{\frac{\left(a_{1}-b_{1}\right)^{2}+\left(a_{2}-b_{2}\right)^{2}+2 a_{2}\left(b_{2}-b_{1}\right)}{a_{1}+a_{2}}\right. \\
& +\frac{\left(c_{1}-b_{1}\right)^{2}+\left(c_{2}-b_{2}\right)^{2}+2 c_{2}\left(b_{2}-b_{1}\right)}{c_{1}+c_{2}} \\
& \left.+\frac{2\left[\left(a_{3}-a_{2}\right)+\left(c_{3}-c_{2}\right)\right] b_{2}+4 b_{1}\left(b_{3}-b_{2}\right)}{b_{2}+2 b_{3}}\right\}
\end{aligned}
$$

Lemma 3.2. If $\rho=R / 3$ and $\delta \leq 1$ and $\delta \leq 1 / 6 \Sigma$ and if

$$
\begin{gathered}
\max \left\{\left|a_{1}-\rho\right|,\left|a_{2}-\rho\right|,\left|a_{3}-\rho\right|,\left|b_{1}-\rho\right|,\left|b_{2}-\rho\right|,\right. \\
\left.\left|b_{3}-\rho\right|,\left|c_{1}-\rho\right|,\left|c_{2}-\rho\right|,\left|c_{3}-\rho\right|\right\} \geq \delta R
\end{gathered}
$$

then

$$
\frac{d}{d t} \ln \frac{\left(b_{2}+2 b_{3}\right)^{2}}{\left(a_{1}+a_{2}\right)\left(c_{1}+c_{2}\right)} \geq c \delta^{2} R
$$

for some constant $c>0$ depending on the initial conditions but independent of $\delta$.

Proof. In the previous Lemma all the terms on the right have the same sign. We have a bound $R \leq \Sigma\left(a_{1}+a_{2}\right)$, so if $\left|a_{1}-b_{1}\right| \geq \delta R$ we have

$$
\frac{\left(a_{1}-b_{1}\right)^{2}}{a_{1}+a_{2}} \geq \frac{\delta^{2}}{\Sigma} R
$$

and the above holds with $c=1 / \Sigma$. Likewise if $\left|a_{2}-b_{2}\right| \geq \delta R$ or $\left|c_{1}-b_{1}\right| \geq \delta R$ or $\left|c_{2}-b_{2}\right| \geq \delta R$. Also if $\left|b_{2}-b_{1}\right| \geq \delta R$ then

$$
\frac{2 a_{2}\left(b_{2}-b_{1}\right)}{a_{1}+a_{2}} \geq \delta R \geq \delta^{2} R
$$

as long as $\delta \leq 1$. If all the above fails, so that

$$
\left|a_{1}-b_{1}\right| \leq \delta R,\left|a_{2}-b_{2}\right| \leq \delta R,\left|b_{2}-b_{1}\right| \leq \delta R
$$

then

$$
b_{2} \geq a_{2}-\delta R \geq \frac{1}{2}\left(a_{1}+a_{2}\right)-\delta R \geq\left(\frac{1}{2 \Sigma}-\delta\right) R
$$

and so if $\delta \leq 1 / 4 \Sigma$ then $b_{2} \geq \frac{1}{4 \Sigma} R$ while if $\delta \leq 1 / 6 \Sigma$

$$
b_{1} \geq b_{2}-\delta R \geq\left(\frac{1}{2 \Sigma}-2 \delta\right) R \geq \frac{1}{6 \Sigma} R .
$$


On the other hand $b_{2}+2 b_{3} \leq 2\left(b_{2}+b_{3}\right)$ and

$$
\left(b_{2}+b_{3}\right)^{2} \leq \Lambda\left(a_{1}+a_{2}\right)\left(c_{1}+c_{2}\right) \leq \Lambda R^{2}
$$

so $b_{2}+b_{3} \leq \Lambda R$ for a constant which is the initial isotropic eccentricity $\Lambda$. Thus for $\delta \leq 1 / 6 \Lambda$

$$
\frac{b_{1}}{b_{2}+2 b_{3}} \geq \frac{1}{6 \Sigma \Lambda}
$$

and we get that the expression on the right in Lemma 2.1 above is at least $c \delta^{2} R$ as claimed.

Next observe

$$
\frac{d}{d t} \ln \left(a_{1}+a_{2}\right) \leq C R \text { and } \frac{d}{d t} \ln \left(c_{1}+c_{2}\right) \leq C R .
$$

for a constant $c$ depending only on $\Sigma$.

Theorem 3.3. For every $\Lambda$ and $\Sigma$ we can find a constant $C$ and $\zeta>0$ such that for every $\delta>0$ there exists an $\alpha>0$ with the property that if we have any complete solution on $0 \leq t<T$ with bounded curvature and eccentricities bounded by $\Lambda$ and $\Sigma$, and if it avoids the exceptional set $E$ where the curvature is necklike and not too small

$$
E=\left\{R(T-t) \geq \zeta \text { and } \max \left\{\left|a_{1}-\rho\right|, \ldots\right\} \leq \delta R\right\}
$$

then

$$
\frac{\left(b_{2}+2 b_{3}\right)^{2}(T-t)^{\alpha}}{\left(a_{1}+a_{2}\right)^{1-\alpha}\left(c_{1}+c_{2}\right)^{1-\alpha}} \leq C \widehat{R}_{0}^{2 \alpha} T^{\alpha}
$$

where $\widehat{R}_{0}=$ maximum of $R$ at $t=0$.

Proof. We have

$\frac{d}{d t} \ln \frac{\left(b_{2}+2 b_{3}\right)^{2}(T-t)^{\alpha}}{\left(a_{1}+a_{2}\right)^{1-\alpha}\left(c_{1}+c_{2}\right)^{1-\alpha}} \leq\left\{\frac{d}{d t} \ln \frac{\left(b_{2}+2 b_{3}\right)^{2}}{\left(a_{1}+a_{2}\right)\left(c_{1}+c_{2}\right)}\right\}-\frac{\alpha}{T-t}+C \alpha R$

for some constant $C$ (depending on $\Lambda$ and $\Sigma$ ). Now if $R(T-t) \leq 1 / C=\zeta$ we are done. Also if $\max \left\{\left|a_{1}-\rho\right|, \ldots\right\} \geq \delta R$ then the first term on the right is $\leq-c \delta^{2} R$ and we are done if $\alpha \leq c \delta^{2} / C$. This shows how to pick $\zeta$ depending on $\Lambda$ and $\Sigma$ only, and $\alpha$ depending on $\Lambda$ and $\Sigma$ and $\delta$. As long 
as we avoid $E$, one way or the other works. Then we only need bound the expression at $t=0$, where

$$
\frac{\left(b_{2}+2 b_{3}\right)^{2}}{\left(a_{1}+a_{2}\right)\left(c_{1}+c_{2}\right)} \leq C
$$

and we are done. Observe that $a_{1}+a_{2}$ and $c_{1}+c_{2}$ are concave functions of the curvature operator $M$, while $b_{3}$ and $b_{2}+b_{3}$ are convex, and hence so is the sum $b_{2}+2 b_{3}$. Thus the set

$$
b_{2}+2 b_{3} \leq C\left(a_{1}+a_{2}\right)^{\frac{1-\alpha}{2}}\left(c_{1}+c_{2}\right)^{\frac{1-\alpha}{2}}\left(\frac{1}{T-t}\right)^{\frac{\alpha}{2}}
$$

is a convex set in $M$ and the function $\frac{1}{T-t}$, which satisfies the evolution

$$
D_{t}\left(\frac{1}{T-t}\right)=\Delta\left(\frac{1}{T-t}\right)+\left(\frac{1}{T-t}\right)^{2}
$$

so we can apply the theorem to the pair $\left(M, \frac{1}{T-t}\right)$ as a system.

If the manifold is compact this completes the proof, using the maximum principle for system explained in [3]. If the manifold is complete with bounded curvature, we can modify the proof along the lines of W.-X. Shi. Since the maximum principle for parabolic equations on complete manifolds is now well understood, we omit the details.

Next we study $a_{3}-a_{1}$. We have

$$
\frac{d}{d t}\left(a_{3}-a_{1}\right) \leq a_{3}^{2}+2 a_{1} a_{2}+b_{3}^{2}-a_{1}^{2}-2 a_{2} a_{3}-b_{1}^{2}
$$

so

$$
\frac{d}{d t} \ln \left(a_{3}-a_{1}\right) \leq a_{3}+a_{1}-2 a_{2}+\frac{b_{3}^{2}-b_{1}^{2}}{a_{3}-a_{1}} .
$$

Now on the set where for some constant $C_{*}$

$$
\frac{\left(a_{3}-a_{1}\right)(T-t)^{\alpha}}{\left(a_{1}+a_{2}\right)^{1-2 \alpha}}=C_{*} \widehat{R}_{0}^{2 \alpha} T^{\alpha}
$$

we have

$$
a_{3}-a_{1}=C_{*} \widehat{R}_{0}^{2 \alpha} T^{\alpha} \frac{\left(a_{1}+a_{2}\right)^{1-2 \alpha}}{(T-t)^{\alpha}} \geq c C_{*} \frac{R^{1-2 \alpha}}{(T-t)^{\alpha}} \widehat{R}_{0}^{2 \alpha} T^{\alpha}
$$


and from Theorem 3.3

$$
b_{3}^{2}-b_{1}^{2} \leq\left(b_{2}+2 b_{3}\right)^{2} \leq C \frac{\left(a_{1}+a_{2}\right)^{1-\alpha}\left(c_{1}+c_{2}\right)^{1-\alpha}}{(T-t)^{\alpha}} \widehat{R}_{0}^{2 \alpha} T^{\alpha}
$$

so

$$
b_{3}^{2}-b_{1}^{2} \leq C \frac{R^{2-2 \alpha}}{(T-t)^{\alpha}} \widehat{R}_{0}^{2 \alpha} T^{\alpha}
$$

This makes

$$
\frac{b_{3}^{2}-b_{1}^{2}}{a_{3}-a_{1}} \leq \frac{C}{C_{*}} R
$$

where $C$ depends only on initial data. Then since

$$
\frac{d}{d t} \ln \left(a_{1}+a_{2}\right) \geq 2 a_{3}+a_{1}
$$

we have

$$
\frac{d}{d t} \ln \frac{a_{3}-a_{1}}{a_{1}+a_{2}} \leq-a_{3}-2 a_{2}+\frac{C}{C_{*}} R \leq-c R+\frac{C}{C_{*}} R
$$

and

$$
\frac{d}{d t} \ln \frac{\left(a_{3}-a_{1}\right)(T-t)^{\alpha}}{\left(a_{1}+a_{2}\right)^{1-2 \alpha}} \leq-c R+C \alpha R+\frac{C}{C_{*}} R-\frac{\alpha}{T-t}
$$

which we can make $\leq 0$ by taking $\alpha$ small compared to some $c$ and $C_{*}$ large compared to some $C$ and discarding the last term.

As before the set

$$
a_{3}-a_{1} \leq C\left(a_{1}+a_{2}\right)^{1-2 \alpha}\left(\frac{1}{T-t}\right)^{\alpha}
$$

is convex and hence preserved by the PDE as long as we avoid $E$. So we get this result.

Theorem 3.4. Under the previous hypothesis are also have

$$
a_{3}-a_{1} \leq C\left(a_{1}+a_{2}\right)^{1-2 \alpha}\left(\frac{1}{T-t}\right)^{\alpha} \widehat{R}_{0}^{2 \alpha} T^{\alpha}
$$

and

$$
c_{3}-c_{1} \leq C\left(c_{1}+c_{2}\right)^{1-2 \alpha}\left(\frac{1}{T-t}\right)^{\alpha} \widehat{R}_{0}^{2 \alpha} T^{\alpha} .
$$


Corollary 3.5. Suppose we have an ancient solution to the Ricci Flow on $-\infty<t \leq 0$ which is complete with bounded curvature and positive isotropic curvature. Suppose the solution is Type $I$, so that $R|t| \leq \Omega$ for some constant $\Omega$, and the isotropic pinching is uniform, so that

$$
|R m| \leq \Gamma\left(a_{1}+a_{2}\right) \quad \text { and } \quad|R m| \leq \Gamma\left(c_{1}+c_{2}\right)
$$

for some constant $\Gamma$ everywhere on the manifold. Then either

(a) the manifold is a compact space form with the shrinking constant positive curvature operator solution, or

(b) there exists $a \zeta>0$ such that for every $\delta>0$ we can find a point $P$ and a time $t$ where

$$
R|t| \geq \zeta \quad \text { and } \max \left\{\left|a_{1}-\rho\right|, \ldots\right\} \leq \delta R
$$

(when $\rho=R / 3$ ).

Proof. By translating time in Theorems 3.3 and 3.4, from $\Gamma$ we can find $\Lambda$ and $\Sigma$ and choose a constant $C$ and $\zeta>0$ such that for every $\delta>0$ either we find a point $P$ and a time $t$ as in (b), or if we avoid this set then we can find $\alpha>0$ such that by 3.3 for every time $t \leq 0$ and every time $\theta \leq t$ we can estimate the curvature pinching at time $t$ by

$$
\frac{\left(b_{2}+2 b_{3}\right)^{2}|t|^{\alpha}}{\left(a_{1}+a_{2}\right)^{1-\alpha}\left(c_{1}+c_{2}\right)^{1-\alpha}} \leq C \widehat{R}_{\theta}^{2 \alpha}|\theta|^{\alpha}
$$

where $\widehat{R}_{\theta}$ is the maximum of $R$ at time $\theta$. Note we translate time so the new time $\theta$ translates to Theorem time 0 , new time $t$ translates to Theorem time $t$, and new time 0 translates to Theorem time $T$. Then in the result in the Theorem we replace $T-t$ by $-t=|t|$ and replace $T=T-0$ by $0-\theta=|\theta|$.] Likewise by 3.4 we get pinching

$$
\frac{\left(a_{3}-a_{1}\right)|t|^{\alpha}}{\left(a_{1}+a_{2}\right)^{1-2 \alpha}} \leq C \widehat{R}_{\theta}^{2 \alpha}|\theta|^{\alpha} \quad \text { and } \quad \frac{\left(c_{3}-c_{1}\right)|t|^{\alpha}}{\left(c_{1}+c_{2}\right)^{1-2 \alpha}} \leq C \widehat{R}_{\theta}^{2 \alpha}|\theta|^{\alpha}
$$

Now by our Type I hypothesis

$$
\widehat{R}_{\theta}^{2 \alpha} \leq \Omega /|\theta|^{2 \alpha}
$$

and since $\theta$ is arbitrarily large, we get $b_{2}+2 b_{3}=0$ and $a_{3}-a_{1}=0$ and $c_{3}-c_{1}=0$. But this forces the manifold to be locally isometric to $S^{4}$, and hence globally a quotient space form $S^{4} / \Gamma$. This is alternative (a). 


\section{The Geometry of Necks.}

\subsection{Harmonic Parametrizations by Spheres.}

Suppose we have a Riemannian manifold $M^{n}$ with metric $g$, and let $S^{n}$ be the sphere with its standard metric $g$. A spherical parametrization of $M^{n}$ is a local diffeomorphism

$$
P: S^{n} \longrightarrow M^{n}
$$

of the sphere to $M$. Let $g$ also denote the pull-back metric of $g$ to $S^{n}$ by $P$. Let $\Gamma$ and $\bar{\Gamma}$ be the connections of $g$ and $\bar{g}$, with covariant derivatives $D$ and $\bar{D}$. We say the spherical parametrization $P$ is harmonic if its Laplacian is zero;

$$
\Delta P=\bar{g}^{i j}\left\{\frac{\partial^{2} F^{\alpha}}{\partial x^{i} \partial x^{j}}-\bar{\Gamma}_{i j}^{k} \frac{\partial F^{\alpha}}{\partial x^{k}}+\Gamma_{\beta \gamma}^{\alpha} \frac{\partial F^{\beta}}{\partial x^{i}} \frac{\partial F^{\gamma}}{\partial x^{j}}\right\}=0
$$

or equivalently on $S^{n}$ if

$$
\bar{g}^{i j}\left\{\Gamma_{i j}^{k}-\bar{\Gamma}_{i j}^{k}\right\}=0 .
$$

We say the parametrization is geometrically $(\varepsilon, k)$ spherical if $g$ is $\varepsilon$-close to $\bar{g}$ on $S^{n}$ in the metric $\bar{g}$

$$
|g-\bar{g}|_{\bar{g}} \leq \varepsilon
$$

and the $j^{\text {th }}$ covariant derivatives $\bar{D}^{j}$ of $g$ are $\varepsilon$-close to zero in the metric $\bar{g}$

$$
\left|\bar{D}^{j} g\right|_{\bar{g}} \leq \varepsilon
$$

for $1 \leq j \leq k$.

Theorem 1.1. If there exists a geometrically $(\varepsilon, k)$ spherical parametrization of $M^{n}$, then there also exists a harmonic spherical parametrization. If $n \geq 3$ it is unique up to a rotation; while for $n=2$ it is unique up to a conformal transformation, and hence unique up to a rotation if we also require that the center of mass of the pull-back metric $g$ on $S^{n} \subseteq R^{n+1}$ lies at the origin 0.

Proof. We look for a harmonic parametrization $P^{*}=P F$ where we want

$$
F:\left(S^{n}, \bar{g}\right) \longrightarrow\left(S^{n}, g\right)
$$


to be harmonic from the standard metric $\bar{g}$ to the pull-back metric $g$. Let $X(F)$ be the vector field on $\left(S^{n}, \bar{g}\right)$

$$
x^{i}=\bar{g}^{i j} g_{\alpha \beta} \Delta F^{\alpha} \frac{\partial F^{\beta}}{\partial x^{j}}
$$

so that for all vector fields $W$ on $\left(S^{n}, \bar{g}\right)$

$$
\langle X, W\rangle_{\bar{g}}=\langle\Delta F, D F(W)\rangle_{g} .
$$

Lemma 1.2. If $V$ is a Killing vector field on $\left(S^{n}, \bar{g}\right)$, i.e. an infinitesimal rotation, then

$$
\int_{S^{n}}\langle X, V\rangle_{\bar{g}} d v(\bar{g})=0
$$

so $X=\mathcal{X}(F)$ is perpendicular to the infinitesimal rotations.

Proof. If $V$ is a Killing vector field

$$
\bar{g}_{i k} \bar{D}_{j} V^{k}+\bar{g}_{j k} \bar{D}_{i} V^{k}=0 \text {. }
$$

The derivative

$$
D F=\left\{\frac{\partial F^{\alpha}}{\partial x^{i}}\right\}
$$

is a section of the bundle of linear maps $L\left(T S^{n}, F^{*} T S^{n}\right)$ of the tangent bundle to the pull-back of the tangent bundle. Let $\nabla_{i}$ denote the covariant derivative on this bundle in the induced connection. Then

$$
\nabla_{i} \frac{\partial F^{\alpha}}{\partial x_{j}}=\nabla_{j} \frac{\partial F^{\alpha}}{\partial x^{i}}
$$

gives a symmetric second derivative, and

$$
\Delta f^{\alpha}=\bar{g}^{i j} \nabla j \frac{\partial F^{\alpha}}{\partial x^{i}} .
$$

The divergence theorem gives

$$
\int \bar{D}_{i}\left\{g_{\alpha \beta} \bar{g}^{i j} \frac{\partial F^{\alpha}}{\partial x^{j}} \frac{\partial F^{\beta}}{\partial x^{k}} V^{k}\right\} d v(\bar{g})=0
$$

which makes

$$
\begin{aligned}
\int g_{\alpha \beta} \Delta F^{\alpha} \frac{\partial F^{\beta}}{\partial x^{k}} V^{k} d v(\bar{g}) & +\int g_{\alpha \beta} \bar{g}^{i j} \frac{\partial F^{\alpha}}{\partial x^{j}} \nabla i \frac{\partial F^{\beta}}{\partial x^{k}} V^{k} d v(\bar{g}) \\
& +\int g_{\alpha \beta} \bar{g}^{i j} \frac{\partial F^{\alpha}}{\partial x^{j}} \frac{\partial F^{\beta}}{\partial x^{k}} \bar{D}_{i} V^{k} d v(g)=0 .
\end{aligned}
$$


If $V$ is Killing then $\bar{g}^{i j} \bar{D}_{i} V^{k}$ is anti-symmetric in $j$ and $k$, so the third term vanishes. But the divergence theorem also gives

$$
\int \bar{D}_{k}\left\{g_{\alpha \beta} \bar{g}^{i j} \frac{\partial F^{\alpha}}{\partial x^{i}} \frac{\partial F^{\beta}}{\partial x^{j}} V^{k}\right\} d v(\bar{g})=0
$$

which makes

$$
2 \int g_{\alpha \beta} \bar{g}^{i j} \frac{\partial F^{\alpha}}{\partial x^{i}} \nabla k \frac{\partial F^{\beta}}{\partial x^{j}} V^{k} d v(\bar{g})+\int g_{\alpha \beta} \bar{g}^{i j} \frac{\partial F^{\alpha}}{\partial x^{i}} \frac{\partial F^{\beta}}{\partial x^{j}} \bar{D}_{k} V^{k} d v(\bar{g})=0
$$

and $\bar{D}_{k} V^{k}=0$ if $V$ is Killing. Thus the second term in the first expansion is zero; and the first term must then be zero also. This proves the Lemma.

Now we return to the proof of the Theorem. Let $M^{2+\alpha}\left(S^{n}, S^{n}\right)$ denote the manifold of $C^{2+\alpha}$ smooth maps of $S^{n}$ to $S^{n}$, let $\mathcal{V}^{\alpha}\left(S^{n}\right)$ denote the space of $C^{\alpha}$ vector fields on $S^{n}$, and let $\mathcal{V}^{\alpha}\left(S^{n}\right)^{\perp}$ denote the subspace orthogonal to the infinitesimal rotations. Then $X=\mathcal{X}(F)$ defines a map

$$
\mathcal{X}: M^{2+\alpha}\left(S^{n}, S^{n}\right) \longrightarrow \mathcal{V}^{\alpha}\left(S^{n}\right)^{\perp}
$$

depending on the metric $g$ as a control parameter. When $g=\bar{g}$ and $F=I$ is the identity, the linearization of $\mathcal{X}$ is the linear operator

$$
D \mathcal{X}: \mathcal{V}^{2+\alpha}\left(S^{n}\right) \longrightarrow \mathcal{V}^{\alpha}\left(S^{n}\right)
$$

where

$$
D \mathcal{X}(V)=\Delta V+(n-1) V .
$$

This clearly has the infinitesimal rotations in its kernel. When $n \geq 3$ the kernel is no larger as can be seen from representation theory, and the image is everything orthogonal to the infinitesimal rotations. Then the inverse function theorem allows us to find a harmonic $F$ near the identity when $g$ is close enough to $\bar{g}$, and $F$ is unique up to a rotation. When $n=2$ the same argument works using infinitesimal conformal transformations. In fact harmonic maps close to the identity are holomorphic, and the result follows from the uniformization theorem.

Corollary 1.3. For every $(\hat{\varepsilon}, \hat{k})$ we can choose $(\varepsilon, k)$ above so that if the original parametrization is geometrically $(\varepsilon, k)$ spherical then the harmonic parametrization is geometrically $(\hat{\varepsilon}, \hat{k})$ spherical. 


\subsection{Geometric Necks.}

We now give precise definitions for several notions of a neck. A topological neck $N$ in a manifold $M^{n}$ is a local diffeomorphism of a cylinder into $M^{n}$ :

$$
N: S^{n-1} \times[a, b] \longrightarrow M^{n} .
$$

Note $N$ need not be globally a diffeomorphism. It will be useful to impose enough conditions on the map $N$ so that it is unique up to an isometry. Let $g$ denote the metric on $M^{n}$, and also its pull-back to the cylinder by $N$; while $\bar{g}$ denotes the standard product metric on the cylinder. We can define the mean radius $r(z)$ of the horizontal sphere $S^{n-1} \times\{z\}$ in the pull-back metric $g$ (restricted to the sphere) so that its area with respect to $g$ is

$$
A\left(S^{n-1} \times\{z\}, g\right)=\sigma_{n-1} r(z)^{n-1}
$$

where $\sigma_{n-1}$ is the area of the unit sphere in the standard metric. We say the topological neck $N$ is normal if it satisfies the following conditions:

- (a) each horizontal sphere $S^{n-1} \times\{z\}$ for $z \in[a, b]$ has constant mean curvature in the pull-back metric $g$;

- (b) the identity map from each horizontal sphere in the standard metric $\bar{g}$ (restricted to the sphere) to the horizontal sphere in the pull-back metric $g$ (restricted to the sphere) is harmonic; and

- $\left(\mathrm{b}^{\prime}\right)$ in the case $n=3$ only, we also require that the center of mass on each $S^{2} \times\{z\}$ in the Euclidean structure on $R^{n} \times\{z\}$ of the mass distribution $d a$ of the pull-back metric $g$ (restricted to the sphere) lies at the origin $\{0\} \times\{z\}$;

- (c) the volume of any subcylinder in the pull-back metric $g$ is given by

$$
V\left(S^{n-1} \times[z, w], g\right)=\sigma_{n-1} \int_{z}^{w} r(z)^{n} d z ;
$$

- (d) if $\bar{V}$ is an infinitesimal rotation on $S^{n-1} \times\{z\}$ (i.e., a Killing vector field in the metric $\bar{g}$ restricted to the sphere) then the $\bar{g}$ average of the $\bar{g}$ inner product of $V$ with the unit vector field $W$ which is $g$ orthonormal to the sphere is zero:

$$
\int_{S^{n-1} \times\{z\}} \bar{g}(V, W) d \bar{a}=0 .
$$


Now we want to make our necks close to a cylinder geometrically. Given $\varepsilon>0$ and an integer $k$, we say that a neck is geometrically $(\varepsilon, k)$ cylindrical if it satisfies the following conditions:

- (A) if $\hat{g}=g / r^{2}$ is the metric on the cylinder conformal to the pull-back metric $g$ modified by the factor $r^{2}$ on each horizontal sphere to have the area of each sphere the same as the unit sphere, then $\hat{g}$ is within $\varepsilon$ of the standard metric $\bar{g}$ :

$$
|\hat{g}-\bar{g}|_{\bar{g}} \leq \varepsilon
$$

- (B) the covariant derivatives $\bar{D}$ of $\hat{g}$ with respect to the standard metric $\bar{g}$ have size at most $\varepsilon$

$$
\left|\bar{D}^{j} \hat{g}\right|_{\bar{g}} \leq \varepsilon
$$

for $1 \leq j \leq k$;

- (C) the logarithm of the mean radius function $r$ has

$$
\left|\left(\frac{d}{d z}\right)^{j} \log r(z)\right| \leq \varepsilon
$$

for $1 \leq j \leq k$.

The set of metrics $g$ on the cylinder that satisfy these conditions form a closed neighborhood of $\bar{g}$, and the collection of all these neighborhoods for all $\varepsilon$ and $k$ defines a basis of the neighborhoods of $\bar{g}$ in the $C^{\infty}$ topology.

Of course to check that we have an $(\varepsilon, k)$ neck it suffices to find a single constant $\bar{r}$ so that if $\check{g}=g / \bar{r}^{2}$ then

$$
|\check{g}-\bar{g}|_{\bar{g}} \leq \varepsilon^{\prime}
$$

and

$$
\left|\bar{D}^{j} \check{g}\right|_{\bar{g}} \leq \varepsilon^{\prime}
$$

for $1 \leq j \leq k^{\prime}$ with suitable $\varepsilon^{\prime}$ and $k^{\prime}$. This definition would appear easier; but it does not work when we wish to combine two overlapping necks into one long one, since we may need a different scaling constant $\bar{r}$ on each neck. This is why we let $r$ be a function of $z$.

We have the following uniqueness result. 
Lemma 2.1. There exists $(\varepsilon, k)$ so that if $N_{1}$ and $N_{2}$ are necks in the same manifold $M^{n}$ and both are normal and geometrically $(\varepsilon, k)$ cylindrical, and if there exists a diffeomorphism $F$ of the cylinders such that $N_{2}=F N_{1}$, then $F$ is an isometry in the standard metrics on the cylinders.

Proof. For any smooth constant mean curvature hypersurface, there exists a unique one-parameter family of nearby constant mean curvature hypersurfaces by the universe function theorem. The map $F$ takes an end of one cylinder to an end of the other. Since these constant mean curvature hypersurfaces agree under $F$, so do all the nearly ones; and we can pursue this all the way from one end to the other. Thus condition (a) guarantees that $F$ preserves the foliation by horizontal spheres. Given the foliation, condition (b) together with the geometric closeness to the standard metric makes $F$ act by isometry on each horizontal sphere $S^{n-1} \times\{z\}$. Condition (c) forces the vertical height functions $z$ to differ by an isometry of $R^{1}$. Then condition (d) forces the isometries of the spheres to align themselves as an isometry of the cylinders; because by (a), (b) and (c) we are dealing with a map of the cylinder to itself which preserves the height and acts on each horizontal sphere by rotation, and if it is perpendicular to the rotations it must be constant.

Now we prove an existence result for normal forms.

Theorem 2.2. For any $\delta>0$ we can find $\varepsilon>0$ and $k$ such that if $N$ is a neck which is geometrically $(\varepsilon, k)$ cylindrical and defined on a cylinder of length at least $3 \delta$ in the standard metric then we can find a normal neck $N^{*}$ and a diffeomorphism $F$ of the domain cylinder of $N^{*}$ onto a region in the domain cylinder of $N$ containing all the points at standard distance at least $\delta$ from the ends, so that $N^{*}=N F$.

Proof. The inverse function theorem guarantees that for any $g$ in a small enough neighborhood of $\bar{g}$ every point at distance at least $\delta$ from the ends lies on a unique constant mean curvature hypersurface which is close to horizontal. Once we have this suitable foliation of a region inside the domain cylinder of $N$, we can choose harmonic parametrizations of the spheres to satisfy (b), choose the height function to satisfy (c), and straighten out the parametrization by rotations of the horizontal spheres to satisfy (d). 
Corollary 2.3. For any $\delta>0$ and any $(\hat{\varepsilon}, \hat{k})$ we can choose $(\varepsilon, k)$ so that the above holds, and the normal neck $N^{*}$ is $(\hat{\varepsilon}, \hat{k})$ cylindrical.

Proof. In the construction above $F$ will be close to the identity by the inverse function theorem.

The next result says that normal necks which are cylindrical enough and overlap more than a little bit near the ends can be combined to form a single long normal neck.

Theorem 2.4. For any $\delta>0$ we can choose $\varepsilon>0$ and $k$ with the following property. If $N_{1}$ and $N_{2}$ are two normal necks in the same manifold $M$ which are both geometrically $(\varepsilon, k)$ cylindrical, and if there is any point $P_{1}$ in the domain cylinder of $N_{1}$ at standard distance at least $\delta$ from the ends whose image in $M$ is also in the image of $N_{2}$, then there exists a normal neck $N$ which is also geometrically $(\varepsilon, k)$ cylindrical, and there exist diffeomorphisms $F_{1}$ and $F_{2}$ such that $N_{1}=N F_{1}$ and $N_{2}=N F_{2}$, provided $n \geq 3$.

Proof. For $n \geq 3$ the sphere $S^{n-1}$ is simply connected, and hence so is the cylinder $S^{n-1} \times[a, b]$. Let $P_{2}$ be a point on the cylinder $N_{2}$ whose image $P=N_{2} P_{2}$ in $M$ is the same as the image $P=N_{1} P_{1}$ of the given $P_{1}$. Suppose $P_{1}$ lies on the sphere $S^{n-1} \times\left\{z_{1}\right\}$ and $P_{2}$ lies on the sphere $S^{n-1} \times\left\{z_{2}\right\}$.

First we claim we can find a map

$$
G: S^{n-1} \times\left\{z_{2}\right\} \longrightarrow S^{n-1} \times\left\{z_{1}\right\}
$$

so that $N_{1} G=N_{2}$ and $G P_{2}=P_{1}$. To see this, take any path $\gamma_{2}$ from $P_{2}$ to any point $Q_{2}$ in $S^{n-1} \times\left\{z_{2}\right\}$. Let $\gamma=N_{2} \gamma_{2}$ be its projection in $M$, and lift $\gamma$ to a path $\gamma_{1}$ in the first cylinder with $\gamma=N_{1} \gamma_{1}$. The point $P_{1}$ is well in the interior, so we can lift the path until we reach a point $Q_{1}$ with $N_{1} Q_{1}=Q=N_{2} Q_{2}$, unless $\gamma_{1}$ runs into the boundary of the first cylinder. But we claim this won't happen because $\gamma_{1}$ is nearly horizontal. The metric $g$ on $M$ pulls back to metrics $g_{1}$ on the domain of $N_{1}$ and $g_{2}$ on the domain of $N_{2}$, each of which are close to the standard metrics $\bar{g}_{1}$ and $\bar{g}_{2}$ on the two cylinders. The horizontal spheres on the cylinders are where the Ricci curvatures of the product metric are all $n-1$, while the vertical direction is where they are 0 . For $k \geq 2$ the curvatures of $g_{1}$ are close to those of $\bar{g}_{1}$, and the curvatures of $g_{2}$ are close to those of $\bar{g}_{2}$. The Ricci curvature of $g_{2}$ 
in the direction of $\gamma_{2}$ is close to $(n-1)$, and the Ricci curvature of $g_{1}$ in the direction of $\gamma_{1}$ is equal to that of $g_{2}$ in the direction of $\gamma_{2}$. Hence $\gamma_{1}$ is close to horizontal. If the path $\gamma_{2}$ is not too long and $(\varepsilon, k)$ are chosen well enough, the path $\gamma_{1}$ cannot exit the cylinder since its length is about the same. Since $S^{n-1}$ is simply connected, the map $G$ taking $Q_{2}$ to $Q_{1}$ is uniquely defined by this process and the choice of $P_{1}$ and $P_{2}$.

Now we claim that the image of $S^{n-1} \times\left\{Z_{2}\right\}$ under $G$ is exactly the sphere $S^{n-1} \times\left\{z_{1}\right\}$, so that $\gamma_{1}$ stayed exactly horizontal. The reason is that the image of $S^{n-1} \times\left\{z_{2}\right\}$ under $G$ will be another constant mean curvature sphere, since locally $G$ extends to an isometry from $g_{2}$ to $g_{1}$, and this new constant mean curvature sphere is nearly horizontal and passes through $P_{1}$. But the inverse function theorem says that such spheres are unique.

The only remaining question is whether the orientations of the normal bundles in the cylinders to the two spheres agree in their images in $M$. If they don't, we can flip one of the cylinders and continue the argument. Then the spheres $S^{n-1} \times\left\{z_{2}+\mu\right\}$ will map to the spheres $S^{n-1} \times\left\{z_{1}+\mu\right\}$ under the obvious extension of $G$ by similar lifts, for $\mu$ near 0 , and hence for $\mu$ in some interval. This lets us patch the cylinders together using $G$, which must be an isometry from $\bar{g}_{2}$ to $\bar{g}_{1}$ by the previous Lemma.

We say a cylinder $N$ is a maximal normal $(\varepsilon, k)$ neck if $N$ is a normal neck which is geometrically $(\varepsilon, k)$ cylindrical, and if whenever $N^{*}$ is another such neck with $N=N^{*} F$ for some $F$ then the map $F$ is onto.

Theorem 2.5. For any $\delta>0$ we can choose $\varepsilon>0$ and $k$ so that any normal neck defined on a cylinder of length at least $3 \delta$ which is geometrically $(\varepsilon, k)$ cylindrical is contained in a maximal normal $(\varepsilon, k)$ neck; or else the target manifold $M$ is diffeomorphic to a quotient of $S^{n-1} \times R^{1}$ by a group of isometries in the standard metric.

Proof. Since the neck $N$ has a domain cylinder of standard length at least $3 \delta$, a point $P$ in the middle has standard distance at least $\delta$ from either end. If there is any other normal neck $N^{*}$ which is geometrically $(\varepsilon, k)$ cylindrical with $N=N^{*} F$ for some $F$, then the previous theorem allows us to extend the definition of $N$ to a longer cylinder, and this extension $\bar{N}$ is unique, and now $N^{*}=\bar{N} \bar{F}$ for a map $\bar{F}$. Take the largest extension $\widetilde{N}$ of $N$. It will be defined on $S^{n-1} \times B^{1}$ for some interval $B^{1} \subseteq R^{1}$. If $B^{1}$ is of the form $[a, b]$ with $-\infty<a<b<\infty$ we have a maximal normal $(\varepsilon, k)$ neck. If we have an interval $(a, b]$ or $[a, b)$ or $(a, b)$ with $-\infty<a<b<\infty$, we have enough bounds to extend the neck to the endpoints, so the original was not 
the largest. If $a=\infty$ but $b<\infty$ or $b=\infty$ but $a<\infty$, then there must be two points $P_{1}$ and $P_{2}$ in the domain cylinder at different heights $z_{1}$ and $z_{2}$ with the same image in $M$, because $M$ has finite volume and $N$ is nearly a local isometry so there must be considerable overlap. In fact we can make $P_{1}$ and $P_{2}$ at least $\delta$ from the finite end. Then the previous theorem shows that the neck $N$ must repeat itself, so both $a=\infty$ and $b=\infty$.

Now if $N: S^{n-1} \times(-\infty, \infty) \longrightarrow M$ is a local diffeomorphism which is geometrically $(\varepsilon, k)$ cylindrical and periodic, its image is open, closed, and connected, and hence all of $M$. Moreover $N$ lifts to the universal cover $\widetilde{M}$ of $M$

$$
\widetilde{N}: S^{n-1} \times(-\infty, \infty) \longrightarrow \widetilde{M}
$$

for $n \geq 3$. Any deck transformation $\gamma: \widetilde{M} \longrightarrow \widetilde{M}$ induces a transformation $\Gamma$ on $S^{n-1} \times(-\infty, \infty)$ with $\widetilde{N} \Gamma=\gamma \tilde{N}$. Arguing as before, we see that $\Gamma$ acts by isometries in the standard metric, since $N=N \Gamma$. This proves the Theorem.

Corollary 2.6. For any $\delta>0$ we can find $\varepsilon>0$ and $k$ so that any two maximal normal $(\epsilon, k)$ necks with lengths at least $3 \delta$ in the standard metrics cannot overlap except within standard distance $\delta$ of their ends.

Proof. Otherwise we could combine them to make a longer neck.

When we perform surgery, we shall do it on maximal $(\varepsilon, k)$ necks of standard lengths at least $3 \delta$, and we shall leave the manifold unchanged in the $\delta$-collars around their ends. This Corollary shows that such surgeries will not interfere with each other.

\subsection{Curvature Necks.}

It is going to be important to detect spheres and necks from their curvature alone. We say the curvature is $\varepsilon$-spherical at $P$ if the curvature operator $R m_{P}$ at $P$ satisfies

$$
\left|R m_{P}-\bar{R} m\right| \leq \varepsilon
$$

where $\bar{R} m$ is the identity curvature operator on the standard round sphere $S^{n}$; if this is true in one orthonormal frame, it is true in every one. We say the curvature is $\varepsilon$-cylindrical at $P$ if there exists an orthonormal frame at $P$ such that

$$
\left|R m_{P}-\bar{R} m\right| \leq \varepsilon
$$


where $\bar{R} m$ is the curvature operator on $S^{n-1} \times R^{1}$ in a standard frame. We say the curvature is $(\varepsilon, k)$ parallel at $P$ if

$$
\left|D^{\ell} R m_{P}\right| \leq \varepsilon \text { for } 1 \leq k \leq \ell .
$$

We say the curvature is $(\varepsilon, k)$ spherical if it is $\varepsilon$-round and $(\varepsilon, k)$ parallel at every point. We say the curvature is $(\varepsilon, k, L)$ cylindrical around $P$ if it is $\varepsilon$-cylindrical and $(\varepsilon, k)$ parallel at every point in the ball of radius $L$ around $P$. We say the curvature is $(\varepsilon, k)$ homothetically spherical or $(\varepsilon, k, L)$ homothetically cylindrical around $P$ if there exists a scaling constant $\sigma$ such that it is true for the scaled metric $\sigma g$. For simplicity, if the curvature is $(\varepsilon, k, L)$ homothetically cylindrical around $P$ we say $P$ lies at the center of an $(\varepsilon, k, L)$ curvature neck.

Theorem 3.1. For every $(\varepsilon, k)$ there exists $\left(\varepsilon^{\prime}, k^{\prime}\right)$ such that if the curvature of a metric is $\left(\varepsilon^{\prime}, k^{\prime}\right)$ spherical then the manifold has a geometrically $(\varepsilon, k)$ spherical parametrization.

Proof. Since the curvature is very pinched near the identity, the Ricci flow will converge by [11]. The convergence is quite rapid, so the limit metric is close to the original one. The limit is a sphere or space form with a round metric of radius almost one. This proves the Theorem.

Theorem 3.2. For every $(\varepsilon, k, L)$ there exists $\left(\varepsilon^{\prime}, k^{\prime}, L^{\prime}\right)$ such that if the curvature is $\left(\varepsilon^{\prime}, k^{\prime}, L^{\prime}\right)$ cylindrical around $P$ then $P$ lies on the center of a geometrically $(\varepsilon, k)$ cylindrical neck $N$ on $S^{n-1} \times[-L, L]$.

Before we prove this Theorem we need a few Lemmas. The strategy is to start by constructing a function which is like the length down the cylinder. Since the curvature at each point in the ball $B_{L^{\prime}}(P)$ of radius $L^{\prime}$ around $P$ is nearly like that on a cylinder, there will be a unique direction, which we call horizontal and write as $\pm H$ where $H$ is a unit tangent vector, where the Ricci curvature $R c(H, H)$ is least. Since the curvature tensor is within $\varepsilon$ of being parallel, the direction $\pm H$ will be a smooth subbundle. Note that it may not be possible to choose an orientation on this bundle in the whole ball, for example if we have a quotient of $S^{n-1} \times[-L, L]$ by a map which acts antipodally on the sphere and flips the interval.

Lemma 3.3. For every $\delta$ there exists $a \lambda$ and an $\left(\varepsilon^{\prime}, k^{\prime}\right)$ such that if we have any minimal geodesic contained in the ball $B_{L^{\prime}}(P)$ of length at least $\lambda$ then the angle it makes at each point with the horizontal is at most $\delta$. 
Proof. For any $\delta$ we can find $\varepsilon^{\prime}$ and $\eta>0$ such that if the curvature operator is within $\varepsilon^{\prime}$ of being cylindrical and if a vector $T$ makes an angle at least $\delta / 2$ with the horizontal $\pm H$ then the Ricci curvature $\operatorname{Rc}(T, T) \geq \eta>0$. Choose $\lambda$ long enough so that Myer's theorem contradicts $R c(T, T) \geq \eta>0$ for the unit tangent $T$ to a geodesic of length $\lambda$. If $\varepsilon^{\prime}$ is small enough and $k^{\prime}$ large enough compared to $\lambda$, then if the angle of the geodesic with the horizontal is at least $\delta$ at any point it will stay at least $\delta / 2$ at every point within distance $\lambda$. This proves the Lemma.

Lemma 3.4. There exists $a \lambda_{0}$ and an $\left(\varepsilon^{\prime}, k^{\prime}\right)$ such that if $\lambda \geq \lambda_{0}$ and we have any minimal geodesic contained in the ball $B_{L^{\prime} / 2}(P)$ from $M$ to $N$ of length at least $6 \lambda$ with midpoint $O$, and if $X$ is any point whose distance from $O$ is at most $\lambda$, then there exists a point $Y$ on the geodesic whose distance from $X$ is at most 4 .

Proof. We can replace 4 by any number greater than $\pi$, the diameter of the sphere. Since $d(O, M)=d(O, N)=3 \lambda$ and $d(O, X) \leq \lambda$, we have $d(X, M) \geq 2 \lambda$ and $d(X, N) \geq 2 \lambda$. Thus the point $Y$ on the geodesic which is closest to $X$ is not an endpoint. A minimal geodesic from $X$ to $Y$ will be perpendicular at $Y$ to the geodesic $M N$. If $\lambda$ is big and $\left(\varepsilon^{\prime}, k^{\prime}\right)$ good the angle between the geodesic $M N$ and $\pm H$ can be made at most $\delta$ for any $\delta>0$ by the previous Lemma. Then the angle between the geodesic $X Y$ and the horizontal $\pm H$ is at least $\pi / 2-\delta$ at $Y$. Since $6 \lambda \leq L^{\prime}, L^{\prime} / 2+\lambda \leq L^{\prime}$ and $X$ is in the ball $B_{L^{\prime}}(P)$ also. If $\left(\varepsilon^{\prime}, k^{\prime}\right)$ are good, the angle with the horizontal will stay at least $\pi / 2-2 \delta$ on all of the geodesic $X Y$. If $\varepsilon^{\prime}$ is small, the Ricci curvature $R c(V, V)$ in the direction of the unit tangent vector $V$ to the geodesic $X Y$ can be kept at least $(1-\theta)(n-1)$ for any $\theta>0$ we like. Then again Myer's theorem gives a contradiction on any length greater than $\pi$ for an appropriate $\theta$. This proves the Lemma.

Lemma 3.5. There exists $a \lambda_{0}$ and an $\left(\varepsilon^{\prime}, k^{\prime}\right)$ such that if $\lambda \geq \lambda_{0}$ and $a$ minimal geodesic from $M$ to $N$ of length at least $6 \lambda$ is contained in the ball $B_{L^{\prime} / 2}(P)$ and if $O$ is its midpoint, and if $X$ is any point at distance at most $\lambda$ from $O$, then a minimal geodesic from $M$ to $X$ and one from $N$ to $X$ arrive at $X$ from opposite sides, one near $H$ and one near $-H$.

Proof. For any $\delta>0$ we can make each minimal geodesic from $M$ or $N$ to $X$ arrive at $X$ at angle within $\delta$ of $\pm H$. Suppose one from $M$ and one from $N$ both arrive close to $+H$ (or both close to $-H$ ). Let $Z$ and $W$ be the points 
on the geodesics $M X$ and $N X$ at distance 5 from $X$. This is possible since $d(M, X) \geq 2 \lambda$ and $d(N, X) \geq 2 \lambda$ and $\lambda$ is big. Since the angle between the geodesics $M X$ and $N X$ at $X$ is at most $2 \delta$ and we can make $\delta$ as small as we like, if we choose $\left(\varepsilon^{\prime}, k^{\prime}\right)$ good enough then we can make $d(Z, W) \leq 1$.

By the triangle inequality

$$
d(M, N) \leq d(M, Z)+d(Z, W)+d(W, N)
$$

and we also have

$$
d(M, Z)=d(M, X)-5 \quad \text { and } \quad d(N, W)=d(N, X)-5
$$

so

$$
d(M, N) \leq d(M, X)+d(N, X)-9 .
$$

Now let $Y$ be the point on the geodesic $L N$ closest to $X$. By the previous Lemma $d(X, Y) \leq 4$. The triangle inequality gives

$$
\begin{aligned}
& d(M, X) \leq d(M, Y)+d(Y, X) \\
& d(N, X) \leq d(N, Y)+d(Y, X)
\end{aligned}
$$

and since $Y$ is on a minimal geodesic

$$
d(M, N)=d(M, Y)+d(Y, N) .
$$

This makes

$$
d(M, N) \geq d(M, X)+d(N, X)-8
$$

and we get a contradiction.

Corollary 3.6. In the ball of radius $\lambda$ around $O$ we can choose an orientation for the horizontal bundle so that at any point $X$ in this ball every minimal geodesic from $M$ to $X$ arrives close to $-H$ and every minimal geodesic from $N$ to $X$ arrives close to $+H$.

Proof. Pick a point $\bar{X}$. Define the orientation in a small neighborhood of $\bar{X}$ so that the criterion is satisfied at $\bar{X}$. Then I claim it also holds at all $X$ in a neighborhood of $\bar{X}$. If not, we can find a sequence of points $X_{j}$ converging to $\bar{X}$ and a sequence of geodesics from $L$ to $X_{j}$ and from $N$ to $X_{j}$ arriving the wrong way around. Since the end points converge, we can find subsequences so that these geodesics converge to a pair of geodesics 
from $L$ to $\bar{X}$ and from $N$ to $\bar{X}$ arriving there the wrong way around. This is a contradiction. Hence the orientation determined by the geodesics from $L$ and $N$ varies continuously. This proves the Corollary.

Now we define a function $f$ in the ball $B_{\lambda}(O)$ which will be approximately the length down the cylinder. We mimic the construction of Jost and Karcher [12]. Let

$$
f(x)=\frac{d(M, X)^{2}-d(N, X)^{2}}{2 d(M, N)} .
$$

Lemma 3.7. The function $f(x)$ is Lipschitz continuous, and smooth almost everywhere. Moreover for any $\gamma>0$ we can choose $\lambda_{0}$ and $\left(\varepsilon^{\prime}, k^{\prime}\right)$ so that if $\lambda \geq \lambda_{0}$ then in $B_{\lambda}(O)$

$$
|D f-H| \leq \gamma
$$

almost everywhere.

Proof. The distance function is Lipschitz continuous, and smooth almost everywhere, since the set of points on the cut locus has zero measure. Suppose $X$ is not on the cut locus of $M$ or $N$. Then the derivative of the distance function $d(M, X)$ from $M$ to $X$ with respect to the variable $X$ is given by the unit tangent vector $T_{M}$ at $X$ of the unique minimal geodesic from $M$ to $X$. For any $\delta>0$, by choosing $\lambda$ and $\left(\varepsilon^{\prime}, k^{\prime}\right)$ we can make

$$
\left|T_{M}-H\right| \leq \delta
$$

Likewise if $T_{N}$ is the unit tangent vector at $X$ of the unique minimal geodesic from $N$ to $X$ then the derivative of the distance function $d(N, X)$ from $N$ to $X$ with respect to the variable $X$ is given by $T_{N}$, and we can make

$$
\left|T_{N}+H\right| \leq \delta .
$$

Now by the chain rule, for any vector $V$

$$
D f \cdot V=\frac{2 d(M, X) T_{M} \cdot V-2 d(N, X) T_{N} \cdot V}{2 d(M, N)}
$$

Therefore

$$
\begin{aligned}
(D f-H) \cdot V & =\frac{d(M, X)\left(T_{M}-H\right) \cdot V-d(N, X)\left(T_{N}+H\right) \cdot V}{d(M, N)} \\
& +\left[\frac{d(M, X)+d(N, X)}{d(M, N)}-1\right] H \cdot V .
\end{aligned}
$$


Now we have seen that the closest point $Y$ to $X$ on the geodesic from $M$ to $N$ is at distance $d(X, Y) \leq 4$. Hence since $d(M, N) \geq 6 \lambda$ we can make

$$
1 \leq \frac{d(M, X)+d(N, X)}{d(M, N)} \leq \frac{6 \lambda+8}{6 \lambda} \leq 1+\psi
$$

for any $\psi>0$ by choosing $\lambda$ large compared to $\psi$. Then

$$
|(D f-H) \cdot V| \leq[(1+\psi) \delta+\psi]|V|
$$

for all $V$, so

$$
|D f-H| \leq(1+\psi) \delta+\psi \leq \gamma
$$

if we make $\psi$ and $\delta$ small compared to $\gamma$. This proves the Lemma.

Corollary 3.8. Under the same assumptions

$$
|f(x)-f(y)| \leq(1+\gamma) d(X, Y)
$$

Proof. We have $|D f| \leq 1+\gamma$ almost everywhere.

Proof. ¡Proof of Theorem 3.2.i Take a geodesic from $P$ to any point $Q$ at distance $L^{\prime}$. (We can do this unless the manifold is contained in the ball of radius $L^{\prime}$ around $P$, in which case the curvature is $\left(\varepsilon^{\prime}, k^{\prime}\right)$ cylindrical everywhere. We deal with this case elsewhere.) Then in a large ball around its midpoint we get a function $f$ and a one-form $H$ such that

$$
D f \approx H \text { and } D H \approx 0
$$

by the previous construction. By the Smoothing Theorem in appendix $\mathrm{F}$ we can find a function $\tilde{f}$ in a slightly smaller ball so that $\tilde{f} \approx f$ and $D \tilde{f} \approx H$ and $D^{2} \tilde{f} \approx 0$. The level sets of $\tilde{f}$ in a slightly smaller ball will be compact (since $\tilde{f}$ is close to $f$ and $f$ has this property) and they will be almost totally geodesic. Hence they have metrics of almost constant curvature. It follows from the sphere-pinching theorem of Huisken [11] that each level set of $\tilde{f}$ is topologically a sphere $S^{n-1}$ or a space form $S^{n-1} / \Gamma$. Moreover we can parametrize one level set by a map of the space form which is nearly an isometry, and extend it to a map of the cylinder $S^{n-1} / \Gamma \times[-L, L]$ into the manifold using the horizontal curves of unit speed along $H$, and this will be nearly local isometry. 
We would like to see that this map is injective. To see this, note that since $D \tilde{f} \approx H$, the rate of change of $\tilde{f}$ along each horizontal curve is nearly 1 . This prevents overlap, since different spheres correspond to different values of $f$.

Now because we have such good bounds on the curvature and its derivatives, we can extend our parametrization to a larger quotient cylinder by again starting at one leaf $S^{n-1} / \Gamma$ where $f$ is constant as before and following out along the direction $H$ with unit speed. The resulting map will be as close as we wish to an isometry, but it may no longer be injective. Nevertheless we can pull the metric on the manifold back to the quotient cylinder using this map, and lift to the cover by $\Gamma$ to get a metric as close as we like to the standard metric on a standard cylinder. Then on a slightly smaller cylinder we can choose a standard parametrization as before with constant mean curvature leaves. This parametrization is unique up to translation and rotation and reflection, and it is invariant under the cover group $\Gamma$, so $\Gamma$ acts by rotations. As a result the induced foliation descends to the quotient cylinder by $\Gamma$. Each quotient sphere $S^{n-1} / \Gamma$ has as its image a constant mean curvature surface which is nearly perpendicular to the vector field $H$. As we take the extension described in the direction back toward our original point $P$, we will stay close to the geodesic from $P$ to $Q$, and hence we will not leave the ball of radius $L^{\prime}$ where we control the curvature until we have gone nearly a distance $L^{\prime}$ back past $P$ in the other direction. In this way we produce a map of the cylinder $S^{n-1} \times[-L, L]$ onto a neighborhood of $P$ which is geometrically $(\varepsilon, k)$ cylindrical. This proves Theorem 3.2.

\subsection{The Fundamental Group.}

Consider a maximal normal geometrically $(\varepsilon, k)$ cylindrical neck

$$
N: S^{n-1} \times[a, b] \rightarrow M^{n} .
$$

The map $N$ may not be one-to-one. This can happen in two distinct ways. First there may be some overlap near the ends

$$
S^{n-1} \times[a, a+\delta] \text { and } S^{n-1} \times[b-\delta, b]
$$

for the $\delta>0$ in Theorem 2.4. This will not concern us, as we have remarked we will not do surgery here. Second, there may be overlap in the middle. But Theorem 2.4 applied to a maximal neck guarantees there is a diffeomorphism

$$
F: S^{n-1} \times[a, b] \rightarrow S^{n-1} \times[a, b]
$$


of the cylinder which induces the overlap, so that $N=N \circ F$. Moreover $F$ must be an isometry in the standard product metric (as well as the pullback metric). The collection of all such diffeomorphisms $F$ form a group $\Gamma$ of isometries of the cylinder in the standard product metric, and the map $N$ descends to a quotient map

$$
N / \Gamma: S^{n-1} \times[a, b] / \Gamma \rightarrow M^{n} .
$$

We call $N / \Gamma$ a geometrically $(\varepsilon, k)$ cylindrical quotient neck.

We can use the hypothesis of positive isotropic curvature to detect necks which are quotients by looking at the fundamental group of the ambient manifold.

Theorem 3.1. There exists $(\varepsilon, k, L)$ such that if a compact manifold $M^{4}$ has a metric of positive isotropic curvature with a geometrically $(\varepsilon, k)$ cylindrical quotient neck

$$
S^{3} \times[-L, L] / \Gamma \longrightarrow M^{4}
$$

then

$$
\pi_{1}\left(S^{3} \times\{0\} / \Gamma\right) \longrightarrow \pi_{1}\left(M^{4}\right)
$$

injects.

First we simplify the problem by considering the question of orientation. Suppose the group $\Gamma$ acts on the cylinder $S^{3} \times[-L, L]$, and the quotient embeds in a manifold $M^{4}$ :

$$
S^{3} \times[-L, L] / \Gamma \longrightarrow M^{4}
$$

Since $\Gamma$ fixes $S^{3} \times\{0\}$ and acts without fixed points, $\Gamma$ preserves the orientation of $S^{3} \times\{0\}$. Let $\Gamma^{+}$be the subgroup which preserves the orientation of the cylinder. If $\Gamma^{+} \neq \Gamma$, we have a diagram of a short exact sequences

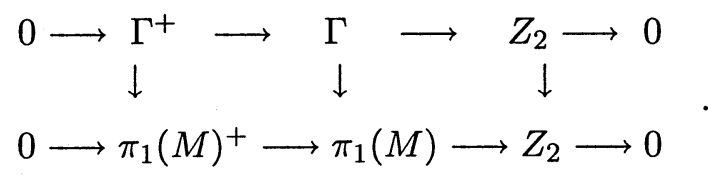

and any kernel of $\Gamma \rightarrow \pi_{1}(M)$ survives in the kernel of $\Gamma^{+} \rightarrow \pi_{1}(M)^{+}$. We can lift to the orientation-preserving cover $M^{+}$which has a neck $S^{n-1} \times$ $[-L, L] / \Gamma^{+}$and show $\pi_{1}$ injects there.

Since the curvature operator of the cylinder is in the interior of the region of positive isotropic curvatures, for $(\epsilon, k, L)$ good enough we can deform the 
metric in the neck so it is the standard metrics (up to a constant factor) in a smaller neck but still has positive isotropic curvature everywhere. This can be close by choosing a bump function $\varphi$ and forming the new metric $\tilde{g}$ from the old metric $g$ and the standard metric $\varphi$ using

$$
\tilde{g}=\varphi g+(1-\varphi) \bar{g} \text {. }
$$

Then

$$
\widetilde{R} m=\varphi R m+(1-\varphi) \bar{R} m+\ldots
$$

where the dots are terms involving derivatives of $R m$ or $\bar{R} m$, which are arbitrarily small.

Lemma 3.2. If $\pi_{1}\left(S^{3} \times\{0\} / \Gamma\right)$ does not inject in $\pi_{1}\left(M^{4}\right)$, then there is a curve $\gamma$ in $S^{3} \times\{0\} / \Gamma$ which does not bound a disk in $S^{3} \times\{0\} / \Gamma$ but does bound a disk in the manifold $M^{4}$ which meets $S^{3} \times\{0\} / \Gamma$ only at $\gamma$, where it is transversal.

Proof. We can find a curve $\gamma$ which does not bound a disc in $S^{3} \times\{0\} / \Gamma$ but does bound a disc $D^{2}$ in $M^{4}$. Choose this disc in general position, so it meets $S^{3} \times\{0\} / \Gamma$ in a collection of curves $\gamma_{i}$ one of which is $\gamma$, with the disc $D^{2}$ transversal to $S^{3} \times\{0\} / \Gamma$ along each $\gamma_{i}$, and with $D^{2}$ crossing itself transversally at isolated points away from the $\gamma_{i}$. The curves $\gamma_{i}$ pull back to smooth disjoint curves $\tilde{\gamma}_{i}$ on the disc $D^{2}$, and the curve $\gamma$ pulls back to the boundary of the disc $\tilde{\gamma}$.

Among all such discs, choose one where the number of curves $\gamma_{i}$ is minimal. One of the curves $\tilde{\gamma}_{*}$ must bound a disc $D_{*}^{2}$ in $D^{2}$. So $\gamma_{*}$ bounds a disc in $M^{4}$. If $\gamma_{*}$ also bounds a disc in $S^{3} \times\{0\} / \Gamma$, we could use this to modify the disc $D^{2}$ to eliminate the intersection curve $\gamma_{*}$ by pulling $\gamma_{*}$ a little off $S^{3} \times\{0\} / \Gamma$ to a neighboring space form $S^{3} \times\{z\} / \Gamma$ and bounding it there. Hence $\gamma_{*}$ does not bound a disc in $S^{3} \times\{0\} / \Gamma$, proving the Lemma.

Now construct a new manifold $\widehat{M}^{4}$ in the following way. Cut $M^{4}$ open along $S^{3} \times\{0\} / \Gamma$ to get a manifold with two boundary components $S^{3} \times\{0\} / \Gamma$, and double across the boundary to get $\widehat{M}^{4}$. Clearly $\widehat{M}^{4}$ also has a metric of positive isotropic curvature, since the boundary is flat extrinsically and we can double the metric. We can take our disk $D_{*}^{2}$ bounding the curve $\gamma_{*}$ and make $D_{*}^{2}$ perpendicular to the boundary in a neighborhood of the boundary, and then double it across one boundary component to get a sphere $S_{*}^{2}$ which is $Z_{2}$ invariant and intersects the boundary component transversally in $\gamma_{*}$. The homotopy class $\left[\gamma_{*}\right]$ is nontrivial in the 
fixed point set component $S^{3} / \Gamma$. Among all spheres which are $Z^{2}$ invariant and intersect the $S^{3} / \Gamma$ in the homotopy class $\left[\gamma_{*}\right] \neq 0$ there will be one of least area. This sphere must even have least area among all nearby spheres. For if a nearby sphere of less area divides in two parts bounding $\left[\gamma_{*}\right]$ in $S^{3} / \Gamma$, one side or the other has less than half the area of the original sphere. We could then double this half to get a sphere of less area which is $Z_{2}$ invariant, contradicting the assumption that ours was of least area among this class. But the hypothesis of positive isotropic curvature implies there are no stable minimal two-spheres as was shown in [13]. Hence we get a contradiction unless $\pi_{1}\left(S^{3} \times\{0\} / \Gamma\right)$ injects in $\pi_{1}\left(M^{4}\right)$.

\subsection{Finding Necks.}

Our previous curvature pinching estimates imply a derivative bound, which we now prove. We obtain it by a compactness argument. There should be a more constructive proof, as in the three-dimensional case with Ricci pinching.

Theorem 5.1. For every $(\varepsilon, k, L)$, every $\theta$ and $\Omega$ and every $\eta$ we can find $\mu$ and $\delta$ and $\sigma$ with the following property. For every $\rho$ and $K$ there exists an $M^{\#}$ so that if $M \geq M^{\#}$ and we have a complete solution to the Ricci Flow with bounded curvature on an interval $0 \leq t \leq T$ for $T \geq \theta / M$ with curvature bounded $|R m| \leq M$ in the ball of radius $\sigma / \sqrt{M}$ around some point $P$ on the time interval and the solution satisfies the estimates

$$
\begin{array}{rll}
a_{1}+\rho>0 & \text { and } & c_{1}+\rho>0 \\
\max \left\{a_{3}, b_{3}, c_{3}\right\} \leq \Omega\left(a_{1}+\rho\right) & \text { and } & \max \left\{a_{3}, b_{3}, c_{3}\right\} \leq \Omega\left(c_{1}+\rho\right)
\end{array}
$$

and

$$
b_{3} \leq(1+\delta) \sqrt{\left(a_{1}+\rho\right)\left(c_{1}+\rho\right)}+K
$$

and if $P$ is a point where

$$
|R m(P, T)| \geq \eta M
$$

and the smallest eigenvalue $\lambda_{1}(P, T)$ of the curvature operator $R m(P, T)$ satisfies

$$
\lambda_{1}(P, t) \leq \mu M
$$

then $P$ lies at the center of an $(\varepsilon, k, L)$ curvature neck. 
Proof. If no such estimate exists, we shall get a contradiction by taking a limit and seeing the limit is cylindrical. Suppose that the estimate fails. Then there exists $\varepsilon, k, L$ and there exists $\theta$ and $\Omega$ and there exists $\eta$ with the following property. For a sequence $\mu_{j} \rightarrow 0$ and $\delta_{j} \rightarrow 0$ there exists $\rho_{j}$ and $K_{j}$ so that for any sequence $M_{j}^{\#}$ we can find a sequence $M_{j} \geq M_{j}^{\#}$ and a sequence of complete solutions to the Ricci flow on intervals $0 \leq t \leq T_{j}$ with $T_{j} \geq \theta / M_{j}$ and with curvature bounded $\left|R m_{j}\right| \leq M_{j}$ in the balls of radius $\sigma / \sqrt{M_{j}}$ around some points $P_{j}$ satisfying

$$
\begin{aligned}
a_{1, j}+\rho_{j}>0 & \text { and } \quad c_{1, j}+\rho_{j}>0 \\
\max \left\{a_{3, j}, b_{3, j}, c_{3, j}\right\} \leq \Omega\left(a_{1, j}+\rho\right) & \text { and } \max \left\{a_{3, j}, b_{3, j}, c_{3, j}\right\} \leq \Omega\left(c_{1, j}+\rho\right)
\end{aligned}
$$

and

$$
b_{3, j} \leq\left(1+\delta_{j}\right) \sqrt{\left(a_{1, j}+\rho\right)\left(c_{1, j}+\rho\right)}+K_{j}
$$

and we can find points $P_{j}$ where

$$
R m_{j}\left(P_{j}, T_{j}\right) \geq \eta M_{j}
$$

and

$$
\lambda_{1, j}\left(P_{j}, T_{j}\right) \leq \mu_{j} M_{j}
$$

and such that $P_{j}$ is not the center of an $(\varepsilon, k, L)$ curvature neck in the metric of the $j^{\text {th }}$ solution at time $T_{j}$. This means we can find points $Q_{j}$ at distance $s_{j} \leq L /\left|R m\left(P_{j}, T_{j}\right)\right|$ where the curvature matrix at time $T_{j}$ scaled by $R m\left(P_{j}, T_{j}\right)$ fails to be within $\varepsilon$ of what it is on the cylinder in any frame or else some covariant derivative of the curvature scaled by $R m\left(P_{j}, T_{j}\right)$ fails to be within $\varepsilon$ of 0 at time $T_{j}$, for some $j \leq k$. Choose a minimal geodesic $\gamma_{j}$ from $P_{j}$ to $Q_{j}$ and choose a frame $F_{j}$ at $P_{j}$ whose first vector $F_{1, j}$ starts in the direction $\gamma_{j}$. (If $P_{j}=Q_{j}$ any frame will do.)

First we get to choose the sequence $M_{j}^{\#}$ to depend on $\rho_{j}$ and $K_{j}$, so we make

$$
\rho_{j} / M_{j}^{\#} \rightarrow 0 \quad \text { and } \quad K_{j} / M_{j}^{\#} \rightarrow 0 .
$$

Then since $M_{j} \geq M_{j}^{\#}$, we know

$$
\rho_{j} / M_{j} \rightarrow 0 \text { and } K_{j} / M_{j} \rightarrow 0 .
$$

Next we dilate the solutions so that $M_{j}$ dilates to $\widetilde{M}_{j}=1$. Then the dilations $\tilde{\rho}_{j}$ of $\rho_{j}$ have $\tilde{\rho}_{j} \rightarrow 0$, and the dilations $\widetilde{K}_{j}$ of $K_{j}$ have $\widetilde{K}_{j} \rightarrow 0$. The times $T_{j}$ dilate to $\widetilde{T}_{j} \geq \theta$, and by translating in time and neglecting the early part we 
can take the dilations to all be defined on $0 \leq t \leq \theta$. They are still complete with dilated curvatures $\widetilde{R} m_{j}$ bounded by $\left|\widetilde{R} m_{j}\right| \leq 1$. We also have

$$
\left|\widetilde{R} m_{j}\left(P_{j}, \theta\right)\right| \geq \eta
$$

and

$$
\tilde{\lambda}_{j}\left(P_{j}, \theta\right) \leq \mu_{j} .
$$

We can now take a limit of these solutions. Since we lack both global curvature bounds and an injectivity radius estimate at $P_{j}$ we may not get a global limit, but we can at least take a local limit of a subsequence in the geodesic tubes out of the $P_{j}$ in the directions $F_{1, j}$ of lengths $2 L / \eta$ in the dilated metrics if $\sigma$ is large compared to $L / \eta$. We choose this since $Q_{j}$ is out along the tube at distance $\tilde{s}_{j} \leq L / \eta$. By choosing another subsequence we can arrange for the $Q_{j}$ to converge to a point $Q$ at distance $\tilde{s} \leq L / \eta$ down the tube.

The limit metric has

$$
a_{1}>0 \text { and } c_{1}>0
$$

since it is not flat, and the limit has

$$
\max \left\{a_{3}, b_{3}, c_{3}\right\} \leq \Omega a_{1} \text { and } \max \left\{a_{3}, b_{3}, c_{3}\right\} \leq \Omega c_{1}
$$

and the limit also has

$$
b_{3} \leq a_{1} c_{1}
$$

since $\delta_{j} \rightarrow 0$ and $\tilde{\rho}_{j} \rightarrow 0$ and $\widetilde{K}_{j} \rightarrow 0$. Moreover we know that in the limit at the point $P=\lim P_{j}$

$$
\lambda_{1}(P, \theta)=0
$$

since $\mu_{j} \rightarrow 0$. Therefore our limit metric has a null eigenvalue for the curvature operator, while the whole operator is non-negative. It follows from [3] that the Lie algebra of the holonomy group is restricted to a proper subalgebra if $\Lambda^{2}$. (Note the strong maximum principle works locally, so we do not need to have a complete solution, one just on the ball will do.) There are not too many possibilities now. Since the limit metric is not flat, holonomy $\{1\}$ is ruled out. Since the limit metric will still have positive isotropic curvature, as in did the pinching $b_{3}^{2} \leq a_{1} c_{1}$ holds, we rule out the Kähler examples of $U(2)$ or $S o(2) \times S o(2)$ or $S o(2) \times\{1\}$ as on $\mathbb{C} P^{2}$ or $S^{2} \times S^{2}$ or $S^{2} \times R^{2}$. The only remaining possibility for the Lie algebra of the holonomy is $S o(3)$. 
Now the only way we get holonomy $S o(3)$ is when in some basis we have $A=B=C$ in the curvature operator matrix, so that

$$
M=\left(\begin{array}{ll}
A & A \\
A & A
\end{array}\right)
$$

which corresponds to the fact that the metric is locally a product of a threedimensional piece with curvature operator $A$ and a one-dimensional piece (which is of course flat). But this makes $a_{1}=b_{1}=c_{1}$ and $a_{2}=b_{2}=c_{2}$ and $a_{3}=b_{3}=c_{3}$, so the inequality $b_{3}^{2} \leq a_{1} c_{1}$ now tells us that $a_{3} \leq a_{1}$. Hence $A$ is a multiple of the identity. Moreover this is true at every point. Now in dimension $>2$, when the curvature is the same in every direction at each point, it must be constant (by the contracted second Bianchi identity). Therefore our limit metric is isometric to an open subset of $S^{3} \times R^{1}$ with the product metric, even at the point $Q$, and the covariant derivatives of the curvature must be zero everywhere, even at $Q$. But at each $Q_{j}$ either we avoid a fixed neighborhood of the multiple of the curvature operator on $S^{3} \times R^{1}$, or some $j^{\text {th }}$ derivative for $j \leq k$ avoids a fixed neighborhood of 0 . By taking a subsequence of the $j$, we can make the same choice happen for each $j$. Then when $j \rightarrow \infty$, the same choice happens at $Q_{j}$ and we get a contradiction. This proves Theorem 5.1.

\section{Surgery.}

\subsection{How to Do Surgery.}

Now we discuss our surgical procedure. We shall perform surgery at certain operating times $0<\tau_{1}<\cdots<\tau_{j}<\ldots$ At each operating time $\tau_{j}$ we perform the following operation, which we call $(\varepsilon, k, L)$ surgery. It consists of performing a standard surgery (which we shall describe) on each maximal normal $(\varepsilon, k)$ neck

$$
N: S^{n-1} \times[a, b] \rightarrow M^{m}
$$

of standard length $b-a \geq L$. We call such a neck an $(\varepsilon, k, L)$ neck. The standard surgery will be invariant under the group of isometries on the cylinder $S^{n-1} \times[a, b]$, and each maximal normal $(\varepsilon, k)$ neck (for a good enough choice of $\varepsilon$ and $k$ ) is uniquely defined up to a rigid motion of the cylinder. It will leave the manifold and the metric unchanged on two collars of standard width $\lambda$ (chosen later) around the ends, i.e.,

$$
S^{n-1} \times[a, a+\lambda] \text { and } S^{n-1} \times[b-\lambda, b]
$$


and (for a good enough choice of $\varepsilon$ and $k$ ) the images of any two distinct maximal $(\varepsilon, k)$ necks are disjoint except possibly for these collars. The surgery will replace $S^{n-1} \times[a, b]$ with two copies of the ball $B_{1}^{n} \cup B_{2}^{n}$. If the neck $N$ is not 1-1 (except possibly on the collars), any multiplicity is represented by a group $\Gamma$ of isometries in both the standard and the pullback metries on the cylinder, so that

$$
N / \Gamma: S^{n-1} \times[a, b] / \Gamma \rightarrow M^{m}
$$

is 1-1 (except possibly on the collars). The group $\Gamma$ will still act by isometries in the new metric on the two balls $B_{1}^{n} \cup B_{2}^{n}$, and we can patch $B_{1}^{n} \cup B_{2}^{n} / \Gamma$ back into $M$. Of course even if the original $M$ has only smooth points, the resulting $M$ may have orbifold points. In fact this happens unless $\Gamma=$ $\{I d\}$, or $\Gamma=Z_{2}$ with the antipodal map $\alpha$ flipping both $S^{n-1}$ and the interval $[a, b]$. In the latter case the surgery on $M^{n}$ removes a copy of $R P^{n}$ as a direct summand. Our assumptions that $n=4$ and $M^{4}$ has positive isotropic curvature, and the only incompressible space forms are $R P^{4}$ 's with unoriented normal bundle, guarantee that surgery produces no orbifold points on $M^{4}$.

Now we discuss the standard surgery. The surgery will take place in four steps. We can describe it at the end near $S^{n-1} \times\{a\}$; and do the same thing at the other end $S^{n-1} \times\{b\}$. For simplicity let us take $a=0$. On the collar

$$
S^{n-1} \times[0, \lambda]
$$

we do nothing. On the collar

$$
S^{n-1} \times[\lambda, 3 \lambda]
$$

we modify the metric conformally by a function $f(z)$ depending only on the height $z$ of each sphere $S^{n-1} \times\{z\}$. This is the most delicate part. The aim is to choose $f$ so that the resulting metric has strictly positive curvature operator on the collar

$$
S^{n-1} \times[2 \lambda, 3 \lambda]
$$

which we do by pinching the neck slightly. We shall discuss this further in a minute. First we finish the description of the standard surgery.

Once the conformally pinched metric $e^{-2 f} g$ has strictly positive curvature operator, we can blend it into the corresponding metric $r_{0}^{2} e^{-2 f} \bar{g}$ obtained by pinching the standard metric $\bar{g}$ in the same way and scaling it by a constant factor $r_{0}$ chosen as the mean radius $r(0)$ at the end of the 
cylinder. We take a suitable bump function $\varphi$ with $\varphi=1$ for $z \leq 2 \lambda$ and $\varphi=0$ for $z \geq 3 \lambda$ and take

$$
\widehat{g}=\varphi e^{-2 f} g+(1-\varphi) r_{0}^{2} e^{-2 f} \bar{g}
$$

as the blended metric. Then $\widehat{g}=e^{-2 f} g$ for $z$ near 2 while $\widehat{g}=r_{0}^{2} e^{-2 f} \bar{g}$ for $z$ near 3.

If we extend the function $f(z)$ suitably with $f(z) \rightarrow 0$ as $z \rightarrow c \leq 4 \lambda$ the resulting metric will be smooth on the ball obtained by adding a point to $S^{n-1} \times[0, c)$. We take this as the metric on the last piece. This completes the surgery. The new metric equals

$$
\left\{\begin{array}{c}
g \text { on } S^{n-1} \times[0, \lambda] \\
e^{-2 f} g \text { on } S^{n-1} \times[\lambda, 2 \lambda] \\
\widehat{g} \text { on } S^{n-1} \times[2 \lambda, 3 \lambda] \\
r_{0}^{2} e^{-2 f} \bar{g} \text { on } S^{n-1} \times[3 \lambda, c]
\end{array}\right.
$$

and extends smoothly to the one-point compactification.

\subsection{Curvature Changes under Surgery.}

We shall show how to pick $\lambda, f$ and $\varphi$ to work for any $\varepsilon$ small enough and $k$ large enough. We also need $L \geq 8 \lambda$ to have enough room between one end of the neck and the other. The crucial part is for $\lambda \leq z \leq 2 \lambda$ where we make the conformal change $\widetilde{g}=e^{-2 f} g$ to pinch the neck a little to give it a positive curvature operator. The basic idea is that the eigenvalues $\lambda$ have their biggest change coming from acquiring a factor $\widetilde{\lambda}=e^{2 f} \lambda$, which doesn't hurt any ratios, while the rest of the change serves to increase the $a_{i}$ 's and $c_{i}$ 's and decrease the $b_{i}$ 's.

Consider then a conformal change of the original metric $g_{i j}$ to a new metric $\widetilde{g}_{i j}$ given by

$$
\tilde{g}_{i j}=e^{-2 f} g_{i j}
$$

Thus lengths are shrunk by a factor $e^{f} \geq 1$ when we take $f \geq 0$. If we take an orthonormal frame $F_{1}, F_{2} . F_{3}, F_{4}$ for $g_{i j}$ then

$$
\widetilde{F}_{a}=e^{f} F_{a}
$$

is an orthonormal frame for $\widetilde{g}_{i j}$. If

$$
F_{a}=F_{a}^{i} \frac{\partial}{\partial x^{i}}
$$


in local coordinates, the metric in this frame is $g_{a b}=g_{i j} F_{a}^{i} F_{b}^{j}=I_{a b}$, and we write the curvature tensor in this frame as

$$
R_{a b c d}=R_{i j k \ell} F_{a}^{i} F_{b}^{j} F_{c}^{k} F_{d}^{\ell}
$$

or equivalently

$$
R_{a b c d}=R m\left(F_{a}, F_{b}, F_{c}, F_{d}\right)
$$

and likewise for the Ricci tensor $R_{a b}$ or the Weyl tensor $W_{a b c d}$. Also we write $\widetilde{R}_{a b c d}$ and $\widetilde{R}_{a b}$ and $\widetilde{W}_{a b c d}$ for the same tensors of the metric $\widetilde{g}_{i j}$ in the frame $\widetilde{F}_{a}$. The following holds in dimension $n$.

Lemma 2.1. The Ricci tensors are related by

$$
\widetilde{R}_{a b}=e^{2 f}\left\{R_{a b}+\Delta f \cdot g_{a b}+(n-2)\left[D_{a} D_{b} f+D_{a} f D_{b} f-|D f|^{2} g_{a b}\right]\right\}
$$

and the Weyl tensors are related by

$$
\widetilde{W}_{a b c d}=e^{2 f} W_{a b c d}
$$

Corollary 2.2. The scalar curvatures are related by

$$
\widetilde{R}=e^{2 f}\left\{R+2(n-1) \Delta f-(n-2)(n-1)|D f|^{2}\right\}
$$

Proof. This is a standard computation, which may be done in local coordinates from

$$
T_{j k}^{i}=\widetilde{\Gamma}_{j k}^{i}-\Gamma_{j k}^{i}=\frac{1}{2} \widetilde{g}^{i \ell}\left(D_{j} \widetilde{g}_{k \ell}-D_{k} \widetilde{g}_{j \ell}-D_{\ell} \widetilde{g}_{j k}\right)
$$

and

$$
\widetilde{R}_{j k \ell}^{i}-R_{j k \ell}^{i}=D_{j} T_{k \ell}^{i}-D_{k} T_{j \ell}^{i}+T_{j m}^{i} T_{k \ell}^{m}-T_{k m}^{i} T_{j \ell}^{m}
$$

and we leave it as an exercise for any reader who has never done it himself or herself. Note as a check that when $n=1$ there is no curvature, and when $n=2$ the Ricci tensor is a multiple of the metric.

When we start the surgery $f$ is small, say $f=c e^{-p /(z-\lambda)}$ where $z$ is the standard distance along the neck and $c$ is a small constant and $p$ is a large one. In this case the second derivative terms $D_{i} D_{j} f$ will surely dominate the quadratic terms $D_{i} f D_{j} f$ in the first derivatives. Remember that since we can make the original metric as close to the product metric of a true 
neck as we wish, we only need a very small positive $f$ to get the neck to look spherical. The dominant part of $D_{i} D_{j} f$ will be in the direction of the neck.

First observe that up to the common factor $e^{2 f}$ the Weyl tensor is unchanged, while the scalar curvature increases. Now the scalar curvature $R$ is the common trace part of the $A$ and $C$ matrices, while the self-dual and anti-self dual parts $W^{+}$and $W^{-}$of the Weyl tensor are the trace-free parts of $A$ and $C$. Therefore all the eigenvalues of $A$ and $C$ will increase, neglecting the common factor $e^{2 f}$. In fact we have

$$
\widetilde{R} \approx e^{2 f}\left\{R+6 D_{4} D_{4} f\right\}
$$

and hence

$$
\tilde{a}_{i} \approx e^{2 f}\left(a_{i}+2 D_{4} D_{4} f\right) \quad \text { for } \quad 1 \leq i \leq 3 .
$$

Now $a_{1} \approx a_{2} \approx a_{3} \approx 1 / r_{0}^{2}$ where $r_{0}$ is the mean radius of the end sphere, while

$$
e^{2 f}-1 \ll \frac{1}{r_{0}^{2}} D_{4} D_{4} f \ll 1
$$

so we get the simpler approximations

$$
\tilde{a}_{i} \approx a_{i}+2 D_{4} D_{4} f \text { for } 1 \leq i \leq 3 .
$$

Likewise

$$
\widetilde{c}_{i} \approx c_{i}+2 D_{4} D_{4} f \text { for } 1 \leq i \leq 3 .
$$

Next we note that we can recover the principal strains of the $B$ matrix from the eigenvalues of the Ricci tensor.

Lemma 2.3. If the Ricci tensor has eigenvalues

$$
\rho_{1} \geq \rho_{2} \geq \rho_{3} \geq \rho_{4}
$$

then the principal strains of the B-matrix are

$$
\begin{aligned}
& b_{1}=\frac{1}{2}\left(\rho_{2}+\rho_{3}-\rho_{1}-\rho_{4}\right) \\
& b_{2}=\frac{1}{2}\left(\rho_{1}+\rho_{3}-\rho_{2}-\rho_{4}\right) \\
& b_{3}=\frac{1}{2}\left(\rho_{1}+\rho_{2}-\rho_{3}-\rho_{4}\right)
\end{aligned}
$$

(up to a constant factor). 
Proof. An orthonormal basis (up to a constant factor) for $\Lambda_{+}^{2}$ and $\Lambda_{-}^{2}$ is given in terms of a frame $F_{1}, F_{2}, F_{3}, F_{4}$ by

$$
\begin{array}{ll}
\varphi_{1}=F_{2} \wedge F_{3}+F_{1} \wedge F_{4} & \psi_{1}=F_{2} \wedge F_{3}-F_{1} \wedge F_{4} \\
\varphi_{2}=F_{3} \wedge F_{1}+F_{2} \wedge F_{4} & \psi_{2}=F_{3} \wedge F_{1}-F_{2} \wedge F_{4} \\
\varphi_{3}=F_{1} \wedge F_{2}+F_{3} \wedge F_{4} & \psi_{3}=F_{1} \wedge F_{2}-F_{3} \wedge F_{4}
\end{array}
$$

and we can compute the entries of the $B$ matrix, which are

$$
B_{11}=B\left(\varphi_{1}, \psi_{1}\right) \quad B_{12}=B\left(\varphi_{1}, \psi_{2}\right) \quad \text { etc. }
$$

to get

$$
\begin{aligned}
& B_{11}=R_{2323}-R_{1414} \\
& B_{22}=R_{3131}-R_{2424} \\
& B_{33}=R_{1212}-R_{3434}
\end{aligned}
$$

and

$$
B_{12}=R_{2331}+R_{1431}-R_{2324}-R_{1424} \text { etc. }
$$

Doing a little algebra we find

$$
\begin{aligned}
& B_{11}=\frac{1}{2}\left(R_{22}+R_{33}-R_{11}-R_{44}\right) \\
& B_{22}=\frac{1}{2}\left(R_{11}+R_{33}-R_{22}-R_{44}\right) \\
& B_{33}=\frac{1}{2}\left(R_{11}+R_{22}-R_{33}-R_{44}\right)
\end{aligned}
$$

while

$$
B_{12}=-\left(R_{12}+R_{34}\right) \text { etc. }
$$

If we choose the frame so that the Ricci tensor is diagonal, then $B$ is diagonal also. If the eigenvalues of the Ricci tensor are $\rho_{1} \geq \rho_{2} \geq \rho_{3} \geq \rho_{4}$ then the principal strains of $B$ are its positive diagonal entries which are given above in increasing order.

Now when the metric is very close to a neck, the first three eigenvalues will be almost equal and quite positive, while the fourth eigenvalue is nearly zero. But in our conformal change the dominant term will be the second derivative $D_{4} D_{4} f$ along the neck. The dominant terms in the change in the Ricci tensor (up to the common factor $e^{2 f}$ ) is thus to add one factor of $D_{4} D_{4} f$ to each term on the diagonal, and an extra two copies $(n-2=2$ if 
$n=4)$ of $D_{4} F_{4} f$ to the last diagonal entry. This has the effect of subtracting two copies of $D_{4} D_{4} f$ from each principal strain of the $B$ matrix. This is the dominant effect, and all other changes resulting from other linear terms $D_{i} D_{j} f$ and quadratic terms $D_{i} f D_{j} f$ can be made small in comparison. Thus all the principal strains of the $B$ matrix will decrease slightly, as desired. We get approximation

$$
\begin{aligned}
& \tilde{b}_{1} \approx e^{2 f}\left(b_{1}-2 D_{4} D_{4} f\right) \\
& \tilde{b}_{2} \approx e^{2 f}\left(b_{2}-2 D_{4} D_{4} f\right) \\
& \widetilde{b}_{3} \approx e^{2 f}\left(b_{3}-2 D_{4} D_{4} f\right) .
\end{aligned}
$$

We get approximations

$$
\tilde{b}_{i} \approx e^{2 f}\left(b_{i}-2 D_{4} D_{4} f\right) \quad \text { for } \quad 1 \leq i \leq 3
$$

which again leads to the easier approximations

$$
\widetilde{b}_{i} \approx-2 D_{4} D_{4} f \text { for } 1 \leq i \leq 3 .
$$

Instead of an approximation it is more rigorous to have estimates. Recall that $z$ is the coordinate down the cylinder $S^{n-1} \times[0,4 \lambda]$ with $z \in[0,4 \lambda]$, and $r_{0}$ is the mean radius of the metric $g$ at $z=0$. Take $f$ to be the function

$$
\left\{\begin{array}{cl}
f(z)=c e^{-p /(z-\lambda)} & \text { for } z>\lambda \\
f(z)=0 & \text { for } 0 \leq z \leq \lambda
\end{array}\right.
$$

where we shall choose appropriate constants $c$ and $p$ soon. This makes

$$
\frac{d f}{d z}=\frac{p}{z^{2}} f \quad \text { and } \quad \frac{d^{2} f}{d z^{2}}=\frac{p(p-2 z)}{z^{4}} f .
$$

Note we always have $f \leq c$. Also since $y=x^{4} e^{-x}$ has its maximum at $x=1 / 4$ where $y \leq 1$, we have

$$
\frac{d^{2} f}{d z^{2}} \leq \frac{c}{p^{2}}\left(\frac{p}{z}\right)^{4} e^{-p / z} \leq \frac{c}{p^{2}} .
$$

If $p \geq 4 \lambda$ then $p-2 z \geq p / 2$ and

$$
\frac{d^{2} f}{d z^{2}} \geq \frac{p^{2}}{2 z^{4}} f
$$

When $0 \leq z \leq \lambda$ we have

$$
f \leq \frac{2 \lambda^{4}}{p^{2}} \frac{d^{2} f}{d z^{2}}
$$


and

$$
\frac{d f}{d z} \leq \frac{2 \lambda^{2}}{p} \frac{d^{2} f}{d z^{2}}
$$

and

$$
\left(\frac{d f}{d z}\right)^{2} \leq 2 c \frac{d^{2} f}{d z^{2}}
$$

and

$$
e^{2 f}-1 \leq 2 e^{2 c} f \leq 2 e^{2 c} \cdot \frac{2 \lambda^{4}}{p^{2}} \frac{d^{2} f}{d z^{2}} .
$$

Now we easily have the following estimates.

Lemma 2.4. For any $\lambda$ and any $\delta>0$ we can choose $c$ small enough and $p$ large enough so that when $f=c e^{-p /(z-\lambda)}$

$$
e^{2 f}-1 \leq \delta \frac{d^{2} f}{d z^{2}} \quad \text { and } \quad \frac{d f}{d z} \leq \delta \frac{d^{2} f}{d z^{2}} \quad \text { and } \quad\left(\frac{d f}{d z}\right)^{2} \leq \delta \frac{d^{2} f}{d z^{2}}
$$

and

$$
\frac{d^{2} f}{d z^{2}} \leq \delta
$$

for $\lambda \leq z \leq 4 \lambda$.

Recall that $r(z)$ is the mean radius of $g$ on the sphere $S^{n-1} \times\{z\}$,

$$
\widehat{g}=g / r^{2}
$$

and $\bar{g}$ is the standard metric, and we have $\widehat{g}$ nearly $\bar{g}$ and $r$ nearly equal to its constant value $r_{0}$ at $z=0$. In particular in an $(\varepsilon, k)$ neck we have

$$
|\widehat{g}-\bar{g}|_{\bar{g}} \leq \varepsilon
$$

and

$$
\left|\bar{D}^{j} \widehat{g}\right|_{\bar{g}} \leq \varepsilon \quad \text { for } \quad 1 \leq j \leq k
$$

and

$$
\left|\left(\frac{d}{d z}\right)^{j} \log r(z)\right| \leq \varepsilon \text { for } 1 \leq j \leq k .
$$

Writing $g=r^{2} \widehat{g}$, we see that for any $k$ and any $\varepsilon^{\prime}$ we can choose $\varepsilon$ above to make

$$
\left|g-r_{0}^{2} \bar{g}\right|_{\bar{g}} \leq \varepsilon^{\prime} r_{0}^{2}
$$


and

$$
\left|\bar{D}^{j} g\right|_{\bar{g}} \leq \varepsilon^{\prime} r_{0}^{2} \quad \text { for } \quad 1 \leq j \leq k .
$$

We choose the frame $\mathcal{F}=\{F a\}$ orthonormal in the metric $g$ so that the Ricci tensor of $g$ is diagonal in this frame, with the eigenvalues $\rho_{1} \geq \rho_{2} \geq \rho_{3} \geq \rho_{4}$. Since $g$ is close to $r_{0}^{2} \bar{g}$,

$$
\rho_{1} \approx \rho_{2} \approx \rho_{3} \approx 2 / r_{0}^{2} \text { and } \rho_{4} \approx 0 .
$$

The covariant derivatives of $z$ in the metric $g$ will be nearly those in the metric $\bar{g}$ since the connections are close. The orthonormal frame $\mathcal{F}=\{F a\}$ in the metric $g$ will be nearly a multiple of an orthonormal frame $\overline{\mathcal{F}}=\left\{\bar{F}_{a}\right\}$ in the metric $\bar{g}$ with $\bar{F}_{4}=\frac{\partial}{\partial z}$

$$
\mathcal{F} \approx \frac{1}{r_{0}} \overline{\mathcal{F}} \quad \text { or } \quad F_{a} \approx \frac{1}{r_{0}} \overline{\mathcal{F}}_{a} .
$$

In particular for any $\varepsilon^{\prime \prime}$ we can find $\varepsilon^{\prime}$ and $k$ above so as to make the covariant derivatives $D_{a} z$ and $D_{a} D_{b} z$ in the orthonormal frame $\mathcal{F}=\left\{F_{a}\right\}$ in the metric $g$ satisfy

$$
\begin{gathered}
\left|D_{4} z-\frac{1}{r_{0}}\right| \leq \frac{\varepsilon^{\prime \prime}}{r_{0}} \\
\left|D_{a} z\right| \leq \frac{\varepsilon^{\prime \prime}}{r_{0}} \text { for } 1 \leq a \leq 3 \\
\left|D_{a} D_{b} z\right| \leq \frac{\varepsilon^{\prime \prime}}{r_{0}^{2}} \text { f or } 1 \leq a, b \leq 4 .
\end{gathered}
$$

Then we can compute the covariant derivatives $D_{a} f$ and $D_{a} D_{b} f$ in the orthonormal frame $\mathcal{F}=\left\{F_{a}\right\}$ in the metric $g$ from

$$
\begin{gathered}
D_{a} f=\frac{d f}{d z} D_{a} z \\
D_{a} D_{b} f=\frac{d f}{d z} D_{a} D_{b} z+\frac{d^{2} f}{d z^{2}} D_{a} z D_{b} z .
\end{gathered}
$$

Given any $\eta$ we can choose $\delta$ and $\varepsilon^{\prime \prime}$ above to get the following result.

Lemma 2.5. For any $\eta>0$ and any $\lambda$ we can choose $\varepsilon$ and $k$ and $c$ small enough and $p$ large enough so that if $f=c e^{-p /(z-\lambda)}$ then

$$
\left|D_{a} f\right| \leq \frac{\eta}{r_{0}} \frac{d^{2} f}{d z^{2}} \quad \text { for } \quad 1 \leq a \leq 4
$$


and

$$
\left|D_{a} D_{b} f\right| \leq \frac{\eta}{r_{0}^{2}} \frac{d^{2} f}{d z^{2}} \quad \text { unless } \quad a=b=4
$$

and

$$
\left|D_{4} D_{4} f-\frac{1}{r_{0}^{2}} \frac{d^{2} f}{d z^{2}}\right| \leq \frac{\eta}{r_{0}^{2}} \frac{d^{2} f}{d z^{2}}
$$

on $\lambda \leq z \leq 4 \lambda$ on any $(\varepsilon, k)$ neck.

Then by the proceeding arguments we can make the following eigenvalue estimates.

Theorem 2.6. For any $\theta>0$ and any $\lambda$ we can choose $\varepsilon$ and $k$ and $c$ small enough and $p$ large enough so that if $f=c e^{-p /(z-\lambda)}$ and $\widetilde{g}=e^{-2 f} g$ then the eigenvalues of the curvature operator of $\tilde{g}$ are related to those of $g$ by

$$
\begin{aligned}
& \left|\widetilde{a}_{i}-\left[a_{i}+2 D_{4} D_{4} f\right]\right| \leq \theta D_{4} D_{4} f \\
& \left|\widetilde{b}_{i}-\left[b_{i}+2 D_{4} D_{4} f\right]\right| \leq \theta D_{4} D_{4} f \\
& \left|\widetilde{c}_{i}-\left[c_{i}+2 D_{4} D_{4} f\right]\right| \leq \theta D_{4} D_{4} f
\end{aligned}
$$

for $1 \leq i \leq 3$ on $\lambda \leq z \leq 4 \lambda$ on any $(\varepsilon, k)$ neck.

Corollary 2.7. We have

$$
\widetilde{a}_{i} \geq a_{i} \quad \text { and } \tilde{b}_{i} \leq b_{i} \text { and } \tilde{c}_{i} \geq c_{i} .
$$

Proof. The previous Lemma lets us make

$$
\left|D_{a} f\right| \leq \eta^{\prime}\left|D_{4} D_{4} f\right| \quad \text { for } \quad 1 \leq a \leq 4
$$

and

$$
\left|D_{a} D_{b} f\right| \leq \eta^{\prime}\left|D_{4} D_{4} f\right| \quad \text { unless } \quad a=b=4
$$

for any $\eta^{\prime}$ we like. Then we can use our curvature formulas to estimate

$$
\begin{aligned}
\left|\widetilde{a}_{i}-e^{2 f}\left[a_{i}+2 D_{4} D_{4} f\right]\right| & \leq \theta^{\prime} D_{4} D_{4} f \\
\left|\tilde{b}_{i}-e^{2 f}\left[b_{i}-2 D_{4} D_{4} f\right]\right| & \leq \theta^{\prime} D_{4} D_{4} f \\
\left|\widetilde{c}_{i}-e^{2 f}\left[c_{i}+2 D_{4} D_{4} f\right]\right| & \leq \theta^{\prime} D_{4} D_{4} f
\end{aligned}
$$


for any $\theta^{\prime}$ we like. Since

$$
a_{i} \approx b_{i} \approx c_{i} \approx 1 / r_{0}^{2}
$$

we can make them all less than $2 / r_{0}^{2}$ easily, and use

$$
e^{2 f}-1 \leq \delta \frac{d^{2} f}{d z^{2}} \leq \frac{\delta}{1-\eta} r_{0}^{2} D_{4} D_{4} f
$$

to expand and get rid of the $e^{2 f}$ in the estimates. The resulting quadratic term in $D_{4} D_{4} f$ can be handled by comparing $r_{0}^{2} D_{4} D_{4} f$ to $d^{2} f / d z^{2}$ and using $d^{2} f / d z^{2} \leq \delta$. In particular

$$
\tilde{a}_{i}-e^{2 f}\left[a_{i}+2 D_{4} D_{4} f\right]=\left\{\widetilde{a}_{i}-\left[a_{i}+2 D_{4} D_{4} f\right]\right\}-\left(e^{2 f}-1\right) a_{i}-2\left(e^{2 f}-1\right) D_{4} D_{4} f
$$

and

$$
\left|\left(e^{2 f}-1\right) a_{i}\right| \leq \frac{2 \delta}{1-\eta} D_{4} D_{4} f
$$

and

$$
\left(e^{2 f}-1\right) D_{4} D_{4} f \leq \frac{\delta^{2}}{1-\eta} D_{4} D_{4} f
$$

gives us what we need. The same works on $\tilde{b}_{i}$ or $\tilde{c}_{i}$. This proves the Theorem.

\subsection{Pinching Estimates under Surgery.}

In the section on curvature pinching estimates we showed that the following estimates are preserved by the Ricci Flow:

$$
\begin{gathered}
a_{1}+a_{2} \geq m \text { and } c_{1}+c_{2} \geq m \\
\left(b_{2}+b_{3}\right)^{2} \leq \Lambda\left(a_{1}+a_{2}\right)\left(c_{1}+c_{2}\right) \\
a_{2}+a_{3} \leq \Phi\left(a_{1}+a_{2}\right) \text { and } c_{2}+c_{3} \leq \Phi\left(c_{1}+c_{2}\right) \\
a_{1}+\rho \geq 0 \text { and } c_{1}+\rho \geq 0 \\
a_{3} \leq \Psi\left(a_{1}+\rho\right) \text { and } c_{3} \leq \Psi\left(c_{1}+\rho\right) \\
b_{3} \leq \Xi e^{P t} \sqrt{\left(a_{1}+\rho\right)\left(c_{1}+\rho\right)} \\
\frac{K b_{3}}{\sqrt{\left(a_{1}+a_{2}\right)\left(c_{1}+c_{2}\right)}} \leq 1+\frac{K}{\max \left\{\ln \sqrt{\left(a_{1}+a_{2}\right)\left(c_{1}+c_{2}\right)}, 2\right\}}
\end{gathered}
$$




$$
\frac{b_{3}}{\sqrt{\left(a_{1}+\rho\right)\left(c_{1}+\rho\right)}} \leq 1+\frac{L e^{P t}}{\max \left\{\ln \sqrt{\left(a_{1}+\rho\right)\left(c_{1}+\rho\right)}, Q\right\}} .
$$

Now we also establish the following.

Theorem 3.1. For a suitable choice of $\varepsilon, k, L, \lambda, f$ and $\varphi$, the above estimates are also preserved by the standard surgery.

Proof. Consider the estimates we need to preserve. Since the $a_{i}$ and $c_{i}$ increase while the $b_{i}$ decrease

$$
\begin{gathered}
a_{1}+a_{2} \geq m \text { and } c_{1}+c_{2} \geq m \\
\left(b_{2}+b_{3}\right)^{2} \leq \Lambda\left(a_{1}+a_{2}\right)\left(c_{1}+c_{2}\right) \\
a_{1}+\rho \geq 0 \text { and } c_{1}+\rho \geq 0 \\
b_{3} \leq \Xi e^{P t} \sqrt{\left(a_{1}+\rho\right)\left(c_{1}+\rho\right)}
\end{gathered}
$$

are all easy. The estimates

$$
\begin{aligned}
& a_{2}+a_{3} \leq \Phi\left(a_{1}+a_{2}\right) \text { and } c_{2}+c_{3} \leq \Phi\left(c_{1}+c_{2}\right) \\
& a_{3} \leq \Psi\left(a_{1}+\rho\right) \quad \text { and } \quad c_{3} \leq \Psi\left(c_{1}+\rho\right)
\end{aligned}
$$

are also easy if $\Phi \geq 2$ and $\Psi \geq 2$, since the $a_{1}, a_{2}, a_{3}$ all increase about the same amount.

For the improved pinching estimates it is easier to see they are preserved if we multiply them out and write them as

$$
2 b_{3} \leq \sqrt{\left(a_{1}+a_{2}\right)\left(c_{1}+c_{2}\right)}\left[1+\frac{K}{\max \left\{\ln \sqrt{\left(a_{1}+a_{2}\right)\left(c_{1}+c_{2}\right)}, 2\right\}}\right]
$$

and

$$
b_{3} \leq \sqrt{\left(a_{1}+\rho\right)\left(c_{1}+\rho\right)}\left[1+\frac{L e^{p t}}{\max \left\{\ln \sqrt{\left(a_{1}+\rho\right)\left(c_{1}+\rho\right)}, Q\right\}}\right]
$$

Now the right hand sides increase if $a_{1}, a_{2}, c_{1}, c_{2}$ increase (at least if $Q \geq 1$ ), since the function

$$
y=\frac{x}{\ln x}
$$

has

$$
y^{\prime}=\frac{\ln x-1}{\ln ^{2} x}
$$


and hence $y$ increases when $\ln x \geq 1$. This shows that all the pinching estimates are preserved by the conformal change on $0 \leq z \leq \lambda$.

If we choose $\varepsilon$ and $k$ good enough, we can make

$$
\begin{aligned}
& \left|a_{i}-\frac{1}{r_{0}^{2}}\right| \leq \frac{\zeta}{r_{0}^{2}} \\
& \left|b_{i}-\frac{1}{r_{0}^{2}}\right| \leq \frac{\zeta}{r_{0}^{2}} \\
& \left|c_{i}-\frac{1}{r_{0}^{2}}\right| \leq \frac{\zeta}{r_{0}^{2}}
\end{aligned}
$$

in an $(\varepsilon, k)$ neck for any $\zeta$ we like. Make $\zeta$ small compared to the minimum size of $d^{2} f / d z^{2}$ on $2 \lambda \leq z \leq 4 \lambda$. Then we can make

$$
a_{i} \geq \frac{1+\zeta}{r_{0}^{2}} \quad \text { and } \quad b_{i} \leq \frac{1-\zeta}{r_{0}^{2}} \quad \text { and } \quad c_{i} \geq \frac{1+\zeta}{r_{0}^{2}}
$$

for $1 \leq i \leq \zeta$ on $2 \lambda \leq z \leq 4 \lambda$. This makes the metric $\tilde{g}=e^{-2 f} g$ have strictly positive curvature operator for $2 \lambda \leq z \leq 4 \lambda$ with a uniform positive lower bound proportional to $\zeta / r_{0}^{2}$.

In the part of the neck where $2 \lambda \leq z \leq 3 \lambda$ we blend the metric $e^{-2 f} g$ into the metric $e^{-2 f} \bar{g}$ with a bump function $\varphi$ and take

$$
\widehat{g}=\varphi e^{-2 f} g+(1-\varphi) \rho_{0}^{2} e^{-2 f} \bar{g}
$$

as our new metric, where $\varphi=1$ for $z \leq 2 \lambda$ and $\varphi=0$ for $z \geq 3 \lambda$. If $(\varepsilon, k)$ are chosen good enough then $g$ is so close to $\bar{g}$ that $\widehat{g}$ is as close as we like to $\rho_{0}^{2} e^{-2 f} \bar{g}$ together with as many derivatives as we like. Thus $\widehat{g}$ also has strictly positive curvature operator and $\widehat{b}_{3} \leq \widehat{a}_{1} \widehat{c}_{1}$. We can now check our curvature pinching estimates on $\widehat{g}$. The estimates

$$
a_{1}+a_{2} \geq m \quad \text { and } \quad c_{1}+c_{2} \geq m
$$

and

$$
a_{1}+\rho \geq 0 \quad \text { and } \quad c_{1}+\rho \geq 0
$$

will hold if we do surgery only on necks with large enough curvature so that $r_{0}$ is small enough. The estimates

$$
a_{2}+a_{3} \leq \Phi\left(a_{1}+a_{2}\right) \quad \text { and } \quad c_{2}+c_{3} \leq \Phi\left(c_{1}+c_{2}\right)
$$


and

$$
a_{3} \leq \Psi\left(a_{1}+\rho\right) \quad \text { and } \quad c_{3} \leq \Psi\left(c_{1}+\rho\right)
$$

hold if $\Phi$ and $\Psi$ are large enough, which is no loss. The remaining estimates hold easily if $b_{3} \leq a_{1} c_{1}$.

Past $z=3 \lambda$ we have the known metric $e^{-2 f} \bar{g}$ on the neck. Now all we have to do is choose $f$ the rest of the way to give a nice end cap, keeping $b_{3} \leq a_{1} c_{1}$. This proves the theorem.

\section{Recovering the Manifold from Surgery.}

\subsection{Operating Procedure.}

We prove the Theorem in the following way. Pick $\gamma>0$ and let $\gamma$ effective $(\varepsilon, k, L)$ surgery be the following procedure. We run the Ricci flow, and perform $(\varepsilon, k, L)$ surgery as described in section D1 at the first time $\tau$ when we can satisfy the following conditions:

- the current maximum of the curvature is at least any previous maximum, and

- after surgery the maximum curvature falls by at least the factor $\gamma$.

The volume $V$ decreases under the Ricci flow, and under surgery. In fact surgery when $R_{M A X}=M$ removes a volume at least $c / M \sqrt{M}$ for some $c>0$. Hence only a finite number of surgeries can happen unless $R$ becomes unbounded. We shall show this cannot happen.

First we need to check that there is indeed a first time we can perform surgery (if there is any time at all when we can). So suppose surgery is possible on standard $(\varepsilon, k, L)$ neck at times $\pi_{j} \searrow \pi$, reducing $R_{\text {MAX }}$ by a factor $\gamma$. We claim such a surgery is also possible at time $\pi$. If not, we shall get a contradiction. Suppose then that $P$ is some point where $R_{P}>\gamma R_{\text {MAX }}$ at time $\pi$ but $P$ cannot be removed by standard surgery at time $\pi$. Then $R_{P}>\gamma R_{M A X}$ for all large enough $j$ by continuity, so $P$ can be surgerically removed at time $\pi_{j}$. Pick $(\varepsilon, k, L)$ necks $N_{j}$ containing $P$ at standard distance at least $\lambda$ from the ends at time $\pi_{j}$. Because of all the estimates we have on the necks, it is easy to check that for a subsequence the necks $N_{j}$ converge to a neck $N$, and $N$ is again an $(\varepsilon, k, L)$ neck at time $\pi$. If we had not chosen the standard parametrization carefully, there would be a problem with the regularity, since from the bound on the $k^{\text {th }}$ derivative 
we only get equicontinuity and hence convergence on the $(k-1)^{s t}$ derivative. However in the standard parametrization each sphere is a minimal surface with a harmonic parametrization, so once we control a few derivatives the regularity theory of elliptic partial differential equations gives bounds on the remaining derivatives. Then our other conditions bound the transverse derivatives as well. Of course we don't get the $k^{\text {th }}$ derivative bounded by $\varepsilon$ from this alone; but since the $k^{\text {th }}$ derivatives in the sequence are all bounded by $\varepsilon$ and they converge uniformly, the $k^{\text {th }}$ derivative of the limit is bounded by $\varepsilon$ also. If each neck $N_{j}$ has standard length at least $L$, so does the limit neck $N$, and $P$ is still at standard distance at least $\lambda$ from the ends. Since the surgeries on $N_{j}$ converge to the surgery on $N$, and since surgery on $N_{j}$ reduces $R$ at $P$ to $\leq \gamma R_{M A X}$, so does surgery on $N$. Hence $P$ can indeed be removed by a standard $(\varepsilon, k, L)$ surgery at time $\pi$ also.

We want to show that if $(\varepsilon, k, L)$ is restrictive enough and $\gamma$ is small enough then we only need to perform a finite number of surgeries to keep $R_{\text {MAX }}$ from going to infinity. This will establish the Main Theorem. Our procedure will be to suppose $R_{M A X} \rightarrow \infty$ for all $(\varepsilon, k, L)$ and all $\gamma$, and obtain a contradiction. The following result will be obtained by a limiting argument.

Theorem 1.1. Suppose $R_{M A X} \rightarrow \infty$ no matter how precisely we choose our surgical parameters. Then there exists an $\eta>0$ such that for any $(\varepsilon, k, L)$ and any $\gamma$ we can construct a complete solution to the Ricci flow with bounded curvature on $0 \leq t \leq 1$ and find a point $P$ in the solution with the following properties:

- (a) the solution has positive curvature operator and satisfies the estimates $a_{3} \leq \Psi\left(a_{1}+\rho\right)$ and $c_{3} \leq \Psi\left(c_{1}+\rho\right)$ and $b_{3}^{2} \leq a_{1} c_{1}$ for the constants $\Psi$ and $\rho$ of section D3

- (b) $R_{M A X}(1) \geq \Gamma$ at $t=1$

- (c) $R(P, 1) \geq \eta$ at $P$ at $t=1$

- (d) $P$ cannot be removed by $(\varepsilon, k, L)$ surgery either by finding $P$ in the part of the neck we remove or by finding $P$ in a ball ending in a neck we remove. 
Proof. We can find a constant $c>0$ so that if $R_{M A X} \leq r_{1}$ at time $t_{1}$ and $R_{M A X} \geq r_{2}$ at time $t_{2}$ then

$$
t_{2}-t_{1} \geq c\left(\frac{1}{r_{1}}-\frac{1}{r_{2}}\right)
$$

as a consequence of the maximum principle. Pick

$$
\gamma=\frac{c}{\Gamma+c}
$$

so that

$$
c\left(\frac{1}{\gamma}-1\right)=\Gamma \text {. }
$$

Also pick $(\widetilde{\varepsilon}, \widetilde{k}, \widetilde{L})$ slightly weaker than $(\tilde{\varepsilon}, k, L)$.

Suppose we perform $\gamma$-effective $(\widetilde{\varepsilon}, \widetilde{k}, \widetilde{L})$ surgeries at times $\tau_{j}$ where $R_{M A X}\left(\tau_{j}\right)=M_{j}$ with $M_{j} \rightarrow \infty$. Pick a subsequence with $M_{j}<M_{j+1}$ and pick times $\theta_{j}$ with $\tau_{j}<\theta_{j}<t_{j+1}$ and $R_{M A X}\left(\theta_{j}\right)=N_{j}$ with $M_{j}<N_{j}<M_{j+1}$. We would have done surgery at time $\theta_{j}$ if we could have reduced $R_{M A X}$ by $\gamma$. Since we did not, there must be some point $P_{j}$ with $R\left(P_{j}, \theta_{j}\right) \geq \gamma R_{M A X}\left(\theta_{j}\right)$ which cannot be removed. Since $R_{M A X}\left(\theta_{j}\right)=N_{j} \geq M_{j}$ and $R_{M A X}\left(\tau_{j}\right)$ dropped from $M_{j}$ to $\gamma M_{j}$, the time elapsed $\theta_{j}-\tau_{j} \geq c(1-\gamma) / \gamma M_{j}$. We can now dilate the solution in space and time and translate in time to get a solution on $0 \leq t \leq 1$ with $R\left(P_{j}, 1\right) \geq \eta$ for some $\eta>0$ independent of $\Gamma$, and where $R_{M A X}(1)=\Gamma$ at time $t=1$. And in this solution $P_{j}$ cannot be surgically removed by $(\varepsilon, k, L)$ surgery. Hence in particular the smallest eigenvalue $\lambda_{1}$ of the curvature operator at $P_{j}$ has a lower bound $\delta>0$ independent of $j$ by Theorems C5.1 and C3.2. Moreover since the curvature pinching improves as $R \rightarrow \infty$ from the estimates in section $B$, we get an injectivity radius estimate by Theorem 25.1 of [10] and the sequence will have a convergent subsequence, and we get a limit which is a solution to the Ricci flow on $0 \leq t \leq 1$ which is complete with bounded curvature and strictly positive curvature operator and has $R_{M A X}=\Gamma$, and there is a point $P$ with $R(P, 1) \geq \eta$ which cannot be removed by $(\varepsilon, k, L)$ surgery since otherwise a close enough metric would allow $(\widetilde{\varepsilon}, \widetilde{k}, \widetilde{L})$ surgery.

Moreover each metric in the sequence satisfies the pinching estimates derived for the Ricci Flow in section B and preserved by the surgery in section $\mathrm{D}$, so we have

$$
a_{3} \leq \Psi\left(a_{1}+\rho\right) \quad \text { and } \quad c_{3} \leq \Psi\left(c_{1}+\rho\right)
$$


and

$$
\frac{b_{3}}{\sqrt{\left(a_{1}+\rho\right)\left(c_{1}+\rho\right)}} \leq 1+\frac{L e^{P t}}{\max \left\{\ln \sqrt{\left(a_{1}+\rho\right)\left(c_{1}+\rho\right)}, Q\right\}}
$$

with the same constants $\Psi, L, P$ and $Q$ for each metric, before we translate and dilate. Also the time $t$ is uniformly bounded $t \leq T<\infty$, so $L e^{P t}$ is also uniformly bounded. Now when the curvature becomes large, $\rho$ and $Q$ are negligible in comparison. After dilating the solution to control the curvature and taking the limit, we see the limit metric satisfies the simple estimates

$$
a_{3} \leq \Psi a_{1} \quad \text { and } \quad c_{3} \leq \Psi c_{1} \quad \text { and } \quad b_{3}^{2} \leq a_{1} c_{1} .
$$

However, this result will be ruled out by the following Enabling Theorem, and we will be done.

\subsection{Enabling Surgery.}

Let $\mathcal{M}$ be the set of solutions to the Ricci flow for $0 \leq t \leq 1$ in dimension 4 which are complete but not compact with bounded curvature and which satisfy the estimates above

$$
a_{3} \leq \Psi a_{1} \quad \text { and } \quad c_{3} \leq \Psi c_{1} \quad \text { and } \quad b_{3}^{2} \leq a_{1} c_{1} .
$$

We then let $\mathcal{M}_{1}$ be the set of metrics $g_{1}$ obtained by evaluating a metric $g_{t}$ in $\mathcal{M}$ at $t=1$. For a metric $g_{1}$ in $\mathcal{M}_{1}$ it is a strong restriction if we require that its maximum scalar curvature $R_{M A X}$ is large, for dilating the solution in space and time makes the curvature less and the duration of the solution longer. Note that topologically $\mathcal{M}_{1}$ if diffeomorphic to $R^{4}$.

It is important to study $\mathcal{M}_{1}$ because if we could not keep $R$ bounded for a compact solution by surgery, we would obtain a limit in $\mathcal{M}_{1}$ with $R_{\text {MAX }}=\Gamma$ while some point $P$ where the scalar curvature $R_{P} \geq \eta$ cannot be removed by surgery, with $\eta>0$ independent of $\Gamma$, by Theorem 1.1.

Theorem 2.1 Enabling Theorem. For every $\eta>0$ and every $(\varepsilon, k, L)$ there exists a $\Gamma$ such that for every metric in $\mathcal{M}_{1}$ with $R_{M A X} \geq \Gamma$ and every point $P$ with $R_{P} \geq \eta$ either

(a) $P$ lies on the center sphere $S^{3}$ if an $(\varepsilon, k, L)$ neck, or

(b) $P$ lies in a differentiable ball $B^{4}$ bounded by the center sphere $S^{3}$ of an $(\varepsilon, k, L)$ neck.

Note that in dimension 4 the Schoenflies Conjecture is still open. Even though the manifold, since it has positive sectional curvature, must be diffeomorphic to $R^{4}$, an embedded $S^{3}$ is not known to necessarily bound a 
differentiable $B^{4}$. Hence this part requires some extra effort, which we address in appendix G.

The proof of Theorem 2.1 will be broken up into a string of Lemmas.

Lemma 2.2. For every $(\varepsilon, k, L)$ and every $\eta$ we can find $\beta>0$ and $\mu<\infty$ with the following property. If we take any metric in $\mathcal{M}_{1}$ and any point $P$ where $R_{P} \geq \eta$, then either

(a) $P$ lies on the center sphere of an $(\varepsilon, k, L)$ neck; or

(b) there exists a point $Q$ where $R_{Q} \geq R_{P}$ and $d(P, Q) \leq \mu / \sqrt{R_{P}}$, and there exists an $r \leq \mu / \sqrt{R_{Q}}$ such that every sectional curvature $K$ at every point in the ball $B_{r}(Q)$ of radius $r$ around $Q$ has $K \geq \beta / r^{2}$.

Proof. First pick $(\bar{\varepsilon}, \bar{k}, \bar{L})$ so that if a point is at the center of an $(\bar{\varepsilon}, \bar{k}, \bar{L})$ curvature neck it is on the center sphere of an $(\varepsilon, k, L)$ geometric neck. Next pick $\mu$ large compared to $\bar{L}$, and rechoose $\bar{\varepsilon}$ even smaller if necessary so $\bar{\varepsilon} \mu$ is much smaller than some constant we shall determine later.

Now let $s_{P}$ denote the distance from $P$, and choose $W_{P}$ as the largest radius for which

$$
R\left(W_{P}-s_{P}\right)^{2} \leq \mu^{2}
$$

everywhere in the ball $s_{P} \leq W_{P}$. Of course

$$
R_{P} W_{P}^{2} \leq \mu^{2}
$$

since $s_{P}=0$ at $P$. Since $W_{P}$ is extremal, there must be some point $Q$ where

$$
R_{Q}\left(W_{P}-s_{P}(Q)\right)^{2}=\mu^{2} .
$$

Let $s_{Q}$ denote the distance of a point from $Q$, and let

$$
W_{Q}=W_{P}-s_{P}(Q) .
$$

By the triangle inequality

$$
W_{Q}-s_{Q} \leq W_{P}-s_{P}
$$

so we have

$$
R_{Q} W_{Q}^{2}=\mu^{2}
$$

and

$$
R\left(W_{Q}-s_{Q}\right)^{2} \leq \mu^{2}
$$

everywhere in the ball $s_{Q} \leq W_{Q}$. 
Now the Harnack inequality [6] tell us that $t R$ is pointwise increasing for a solution in $\mathcal{M}$, so the value of $R$ at a point at a time $1 / 2 \leq t \leq 1$ is no more than twice its value at $t=1$. This time interval is a fraction of $1 / \eta$, depending on $\eta$. This gives estimates on $R$ in a ball around $Q$ of radius $W_{Q} / 2$ for $1 / 2 \leq t \leq 1$. Then the strong maximum principle tells us as in Theorem C5.1 that there exists a $\delta>0$ such that either all sectional curvatures $K$ at $Q$ are at least $\delta R_{Q}$, or else $Q$ is the center of an $(\bar{\varepsilon}, \bar{k}, \bar{L})$ curvature neck, as long as we choose $\mu$ large compared to $\bar{L}$.

In the first case, since we have estimates depending on $\eta$ on the derivatives of the curvature in a smaller ball around $Q$ of size $W_{Q} / 3$, we easily find that $Q$ is the center of a bump of radius $r=W_{Q} / 3$ where $K \geq \beta / r^{2}$ for an appropriate $\beta$. Now

$$
d(P, Q) \leq W_{P} \leq \mu / \sqrt{R_{P}}
$$

so $Q$ is close to $P$ as desired, and $r \leq W_{Q}=\mu / \sqrt{R_{Q}}$. In the second case, we claim that $Q=P$, and hence $P$ lies on the center sphere of an $(\varepsilon, k, L)$ neck.

For if $Q \neq P$, choose a minimal length geodesic from $P$ to $Q$. Extend it past $Q$ and call the distance from $P$ along the geodesic $s$. Then $s_{P}=s$ up to $Q$, and $s_{P} \leq s$ past $Q$. It follows that $R\left(W_{P}-s\right)^{2}$ also has a maximum value equal to $\mu^{2}$ at $s=s_{P}(Q)=d(P, Q)$, and its first derivative there must be zero. This gives us the equation

$$
\frac{d}{d s} R_{Q}=2 R_{Q} / W_{Q}=\frac{2}{\mu} R_{Q}^{3 / 2} .
$$

But in an $(\bar{\varepsilon}, \bar{k}, \bar{L})$ neck we have

$$
\frac{\partial}{\partial s} R_{Q} \leq C \bar{\varepsilon} R_{Q}^{3 / 2}
$$

for some constant $C$. If $\bar{\epsilon}$ is so small that $\bar{\varepsilon} \mu$ is smaller than $1 / C$ we get a contradiction. This means $Q$ must be $P$, where we do not get such an argument on the derivative since $s_{P}$ is not differentiable.

Lemma 2.3. For every $\theta$ we can find $(\varepsilon, k, L)$ such that if two minimal geodesics go from the same point on one end of an $(\varepsilon, k, L)$ neck to the other end, then the angle between them at the common endpoint is $\theta$ or less.

Proof. First observe that it is true on a standard cylinder of radius 1 if the length $L$ is great enough. This is because a geodesic in the product metric 
projects to a geodesic in each factor, and if it travels further than $\pi$ in the sphere it does not minimize distance. Consequently the angle a minimal geodesic down the cylinder makes with a straight line down the cylinder is no more than $\operatorname{arc} \tan (\pi / L)$; and the angle between two geodesics is no more than twice this. So it suffices to take $L>\pi / \tan (\theta / 2)$.

Suppose now that the metric is not the standard one, but is very close to it. A geodesic has constant velocity, and its components on the sphere and the interval will be nearly constant. Again if the sphere component of the velocity is too large compared to the interval component, the second variation formula for arc length becomes negative, and the geodesic cannot be minimal. But if two minimal geodesics of speed one have small sphere components to their velocity, the angle between them is small. This proves the Lemma.

Lemma 2.4. For every $(\varepsilon, k, L)$ and every $\eta$ we can find $(\widehat{\varepsilon}, \widehat{k}, \widehat{L})$ with the following property. If we take any metric in $\mathcal{M}_{1}$ which has an $(\widehat{\varepsilon}, \widehat{k}, \widehat{L})$ neck with $R \leq \eta$ somewhere on its center sphere, and $P$ is a point outside the center sphere of the neck on the non-compact side where $R_{P} \geq \eta$, then $P$ lies on the center sphere if an $(\varepsilon, k, L)$ neck.

Proof. Given $(\varepsilon, k, L)$ and $\eta$, choose $\beta$ and $\mu$ as in Lemma 2. Now suppose we have a metric in $\mathcal{M}_{1}$ and a point $P$ where $R_{P} \geq \eta$, but $P$ is outside the center sphere of an $(\varepsilon, k, L)$ neck. Then, as in the Lemma, we can find a point $Q$ where $R_{Q} \geq R_{P} \geq \eta$ and $d(P, Q) \leq \mu / \sqrt{R_{P}}$, and we can find $r \leq \mu / \sqrt{R_{Q}}$ with $K \geq \beta / r^{2}$ in $B_{r}(Q)$.

Now by the Bump Avoidance Theorem 21.4 in [10], we can chose $\lambda$ depending only on $\beta$, so that if we take any minimal geodesic between points $X$ and $Q^{\prime}$ both at distance at least $s$ from $Q$ with $s \geq \lambda r$, then the entire geodesic $X Q^{\prime}$ stays away from $Q$ by a distance at least $s / \lambda$. Choose $\theta$ with $\tan \theta<1 / \lambda$. We then pick $(\widehat{\varepsilon}, \widehat{k}, \widehat{L})$ so that $\widehat{L}$ is large compared to $\lambda$ and $\mu$, and so that any two minimal geodesics from a common endpoint at one end of an $(\widehat{\varepsilon}, \widehat{k}, \widehat{L})$ cylinder to the other end make an angle at most $\theta$, which we can do by Lemma 3.

Suppose now that the point $P$ lies on the outside of the center sphere of an $(\widehat{\varepsilon}, \widehat{k}, \widehat{L})$ neck where some point on the center sphere has $R \leq \eta$. Then the radius of the neck is at least $1 / \sqrt{\eta}$ or not much less, and the length is at least $\widehat{L} / \sqrt{\eta}$ or not much less. Pick a smaller $(\widehat{\varepsilon}, \widehat{k}, \widehat{L} / 6)$ neck in the bigger neck, going from (roughly will do) one-sixth to one-third of the way from the 
inner end to the outer end. Since we have left a collar of length at least or not much less than $\widehat{L} / 6 \sqrt{\eta}$, and $d(P, Q) \leq \mu / \sqrt{R_{P}} \leq \mu / \sqrt{\eta}$, and $\widehat{L}$ is large compared to $\mu$, and $P$ is outside the center sphere, $Q$ is still outside the small neck. Then take a point $X$ on the inner end of the smaller cylinder, and connect it to $Q$ by a minimal geodesic $X Q$. It is clearly inefficient for the geodesic to backtrack out of the inner end of the big cylinder (since we have left a long collar on that end also), and therefore it must proceed monotonically down the small cylinder, exiting at a point $Y$ on the outer end of the small cylinder.

Likewise if we pick a very remote point $Q^{\prime}$ and connect it by a minimal geodesic to $X$, we get another minimal geodesic going monotonely down the small cylinder from $X$ on the inner end to some point $Z$ on the outer end, and then on to $Q^{\prime}$.

Since the distance from $X$ to $Q$ or $Q^{\prime}$ is at least $\widehat{L} / 6 \sqrt{\eta}$ or not much less, while the radius $r$ of the curvature bump at $Q$ has $r \leq \mu / \sqrt{\eta}$, and $\widehat{L}$ is large compared to $\lambda \mu$, we can make the distance $s$ from $X$ to $Q$ at least $\lambda r$. Similarly if $Q^{\prime}$ is remote we can make the distance from $Q$ to $Q^{\prime}$ at least $s$ and the distance from $X$ to $Q^{\prime}$ at least $s$ also.

Pick the point $Q^{\prime \prime}$ along the geodesic from $X$ to $Q^{\prime}$ at distance exactly $s$ from $X$. By the Bump Avoidance Lemma, the distance from $Q$ to $Q^{\prime \prime}$ is at least $s / \lambda$. On the other hand, the angle between $X Q$ and $X Q^{\prime \prime}$ is the angle between $X Y$ and $X Z$, which is at most $\theta<\arctan 1 / \lambda$. Since the sectional curvatures are positive, we can use Toponogov's Theorem (see[1]) to conclude that the distance from $Q$ to $Q^{\prime \prime}$ is at most the base of a Euclidean isosceles triangle with two equal sides $s$ and included angle $\theta$. The base has length $2 s \tan \theta / 2 \leq s \tan \theta<s / \lambda$, and we have a contradiction. This proves the Lemma.

Lemma 2.5. For every $(\varepsilon, k, L)$ and every $\eta$ we can find an $\left(\varepsilon^{\prime}, k^{\prime}, L^{\prime}\right)$ with the following property. If a metric in $\mathcal{M}_{1}$ has an $\left(\varepsilon^{\prime}, k^{\prime}, L^{\prime}\right)$ neck, then every point $P$ outside its center sphere on the non-compact side where $R_{P} \geq \eta$ lies on the center sphere of an $(\varepsilon, k, L)$ neck.

Proof. Given $(\varepsilon, k, L)$ and $\eta$, choose $(\widehat{\varepsilon}, \widehat{k}, \widehat{L})$ as in Lemma 4 , and first require $\left(\varepsilon^{\prime}, k^{\prime}, L^{\prime}\right)$ to be at least as restrictive. Now if a metric in $\mathcal{M}_{1}$ has an $\left(\varepsilon^{\prime}, k^{\prime}, L^{\prime}\right)$ neck it is also an $(\widehat{\varepsilon}, \widehat{k}, \widehat{L})$ neck, and if $R \leq \eta$ somewhere on its center sphere we are done by Lemma 4 .

Otherwise we have an $\left(\varepsilon^{\prime}, k^{\prime}, L^{\prime}\right)$ neck with $R \geq \eta$ everywhere on its center sphere. Again we construct a smaller $\left(\varepsilon^{\prime}, k^{\prime}, L^{\prime} / 6\right)$ neck going from 
(roughly) one-sixth to one-third of the way from the inner end. Suppose now that $P$ is a point beyond the center sphere of the $\left(\varepsilon^{\prime}, k^{\prime}, L^{\prime}\right)$ neck where $R_{P} \geq \eta$. Connect $P$ by a minimal geodesic to a point $X$ on the inner end of the smaller $\left(\varepsilon^{\prime}, k^{\prime}, L^{\prime} / 6\right)$ cylinder. Let $\widetilde{P}$ be the last point going from $X$ to $P$ such that $R_{\widetilde{P}} \geq \eta$ and $\widetilde{P}$ is the center of an $(\widehat{\varepsilon}, \widehat{k}, \widehat{L})$ neck. Since $\left(\varepsilon^{\prime}, k^{\prime}, L^{\prime} / 6\right)$ can be chosen more restrictive than $(\widehat{\varepsilon}, \widehat{k}, \widehat{L})$, we can be sure $\widetilde{P}$ is beyond the center sphere of the bigger $\left(\varepsilon^{\prime}, k^{\prime}, L^{\prime}\right)$ neck. We are guaranteed the existence of a furthest $\widetilde{P}$ because a limit of necks in standard parametrization is a neck. If $\widetilde{P}=P$ we are done. If $R_{\widetilde{P}} \leq \eta$ we are done by Lemma 4 , since $P$ is outside the center sphere of the $(\widehat{\varepsilon}, \widehat{k}, \widehat{L})$ neck at $\widetilde{P}$. (Indeed we get one long neckline region from well before $X$ all the way to well past $P$, as the individual $(\widehat{\varepsilon}, \widehat{k}, \widehat{L})$ necks fit together, and the geodesic from $X$ to $\widetilde{P}$ must be nearly along the lateral direction since it is so long; and since the necklike region extends well past $\widetilde{P}$, if $P$ were back before the center sphere of the neck at $\widetilde{P}$ our path would not be the shortest from $X$ to $P$.) And if $P \neq \widetilde{P}$ and $R_{\widetilde{P}}>\eta$ and $\widetilde{P}$ is furthest, some point $P^{\#}$ just past $\widetilde{P}$ (but as close as we like) is not on the center sphere of an $(\widehat{\varepsilon}, \widehat{k}, \widehat{L})$ neck but has $R_{P \#} \geq \eta$ still.

Let us pause to compare the curvature at $\widetilde{P}$ with its distance $s$ from $X$.

Lemma 2.6 Sublemma. For every $(\widehat{\varepsilon}, \widehat{k}, \widehat{L})$ sufficiently restrictive and every $\nu<\infty$ we can find $\left(\varepsilon^{\prime}, k^{\prime}, L^{\prime}\right)$ so that $s \geq \nu / \sqrt{R_{\widetilde{P}}}$.

Proof. Take a point $\stackrel{\sim}{P}$ on the center sphere through $\widetilde{P}$ of the $(\widehat{\varepsilon}, \widehat{k}, \widehat{L})$ neck there so that $\tilde{P}$ is (roughly) antipodal to $\widetilde{P}$. Then the distance from $\widetilde{P}$ to $\tilde{P}$ is (roughly) $\pi / \sqrt{R_{\tilde{P}}}$. Connect $\tilde{P}$ to $X$ by another minimal geodesic. By choosing $\left(\varepsilon^{\prime}, k^{\prime}, L^{\prime}\right)$ with $L^{\prime}$ large enough we can make the angle $\theta$ between $X \widetilde{P}$ and $X \widetilde{P}$ as small as we like. If $s$ is the distance from $X$ to $\widetilde{P}$, the distance from $X$ to $\tilde{P}$ is within (roughly) $\pi / \sqrt{R_{\widetilde{P}}}$ of $s$. As long as $\widehat{L}$ is reasonably long, we can replace $\widetilde{P}$ and $\tilde{P}$ by points $\widetilde{P}^{\prime}$ and $\tilde{P}^{\prime}$ near them along the geodesics, no more that $\pi / \sqrt{R_{P}}$ away, so that the distance between $\widetilde{P}^{\prime}$ and $\tilde{P}^{\prime}$ is still nearly $\pi / \sqrt{R_{P}}$, so at least $1 / \sqrt{R_{P}}$, and the distance from $X$ to $\widetilde{P}^{\prime}$ and $\tilde{P}^{\prime}$ are both equal to some $s^{\prime} \leq s$. Now comparing to an isosceles Euclidean triangle with two sides $s^{\prime}$ and included angle $\theta$, we see 
the distance from $\widetilde{P}^{\prime}$ to $\stackrel{\sim \prime}{P}^{\prime}$ is at most $s^{\prime} \tan \theta(\operatorname{since} 2 \sin (\theta / 2) \leq \tan \theta)$. Then

$$
s^{\prime} \tan \theta \geq 1 / \sqrt{R_{P}}
$$

and we only need to make $\tan \theta \leq 1 / \nu$ to prove Lemma 6 .

We can now return to the proof of Lemma 2.5. If we apply Lemma 2.2 at the point $P^{\#}$, we find $\beta$ and $\mu$ depending on $(\widehat{\varepsilon}, \widehat{k}, \widehat{L})$ and $\eta$, and we find a point $Q$ and a radius $r$ with $R_{Q} \geq R_{P \#}$ and

$$
d\left(P^{\#}, Q\right) \leq \mu / \sqrt{R_{P^{\#}}} \quad \text { and } \quad r \leq \mu / \sqrt{R_{Q}}
$$

such that every sectional curvature $K$ at every point in $B_{r}(Q)$ has $K \geq \beta / r^{2}$. We can then find $\lambda$ depending on $\beta$ from the Bump Avoidance Lemma so that any minimal geodesic between points at distance at least $s \geq \lambda r$ avoids $Q$ by a distance at least $s / \lambda$.

Now $d\left(X, P^{\#}\right)=s \geq \nu / \sqrt{R_{\tilde{P}}}$ while $d\left(P^{\#}, Q\right) \leq \mu / \sqrt{R_{P \#}}$ and $R_{P \#}$ is nearly $R_{P}$. Since we can make $\nu$ very big compared to $\mu, d(X, Q)$ is nearly $d\left(X, P^{\#}\right)$. Now $P^{\#}$ is beyond the center sphere of the big $\left(\varepsilon^{\prime}, k^{\prime}, L^{\prime}\right)$ neck, so this suffices to place $Q$ outside the smaller $\left(\varepsilon^{\prime}, k^{\prime}, L^{\prime} / 6\right)$ neck. Moreover $r \leq \mu / \sqrt{R_{Q}}$ and $R_{Q} \geq R_{P \#}$ and $R_{P \#}$ is nearly $R_{\tilde{P}}$, so $r$ is not much more than $\mu / \sqrt{R_{\tilde{P}}}$; and by making $\nu$ large compared to $\lambda \mu$, we can make $s \geq \lambda r$. Now as in the proof of Lemma 2.4, if we connect $X$ to a very distant point $Q^{\prime}$ by a minimal geodesic and pick $Q^{\prime \prime}$ along it with the same distance $s$ from $X$ as $Q$, and make the angle between $X Q$ and $X Q^{\prime \prime}$ very small by making $\left(\varepsilon^{\prime}, k^{\prime}, L^{\prime}\right)$ very good, we get a contradiction. This proves Lemma 2.5. Now we just need to show we can find as good a neck as we take by making $R_{M A X}$ large at $t=1$.

Lemma 2.7. For all $\eta$ we can find a $\delta$ with the following property. Given any solution to the Ricci flow in $\mathcal{M}_{1}$ with $R_{M A X} \geq \eta$, we can find a point $P$ where $R_{P} \geq \frac{1}{2} R_{M A X}$ and the smallest eigenvalue of the curvature operator at $P$ satisfies $\lambda_{1, P} \geq \delta R_{M A X}$.

Proof. Given $\eta$ and a decent $(\varepsilon, k, L)$ we can find $\delta$ so that if we have a solution in $\mathcal{M}_{1}$ with $R_{M A X} \geq \eta$ and a point $P$ where $R_{P} \geq \frac{1}{2} R_{M A X}$ and where $\lambda_{1, P} \leq \delta R_{M A X}$, then $P$ lies on the center sphere of an $(\varepsilon, k, L)$ neck. Suppose every point $P$ where $R_{P} \geq \frac{1}{2} R_{\text {MAX }}$ has $\lambda_{1, P} \leq \delta R_{M A X}$, which is true if the Lemma fails. Choose a point $Q$ where $R_{Q} \geq \frac{3}{4} R_{M A X}$. Then 
$R$ is nearly as large near $Q$, so the $(\varepsilon, k, L)$ neck centered at $Q$ extends in both directions to form a long neck. Each point in the middle of the long neck will be on the center sphere of an $(\varepsilon, k, L)$ neck, out at least until we get points $X$ on one side and $Y$ on the other where $R_{X}=R_{Y}=\frac{1}{2} R_{M A X}$. Moreover this neck will be foliated by constant mean curvature spheres. For a good $(\varepsilon, k, L)$ the area of the spheres through $X$ and $Y$ must be a bit larger than the area of the sphere through $Q$. Hence there will be some sphere in the middle where the area is no more than its neighbors. Since the rate of change of the area is zero, this sphere will be a minimal sphere.

We claim it is a stable minimal sphere, in the sense that all nearby spheres have at least as much area. To see this, think of any nearby sphere as a graph in the parametrization $S^{3} \times B^{1}$. At any constant graph the second variation is strictly positive in the directions orthogonal to the constants. (This is clearly true in the product metric, and hence in any metric close enough to the product.) Then we can accomplish any small variation by first moving by a constant, which does not decrease area, and then moving orthogonal to the constants, which increases area. This shows the minimal sphere is stable. But since metrics in $\mathcal{M}_{1}$ have strictly positive curvature operator, the second variation in the normal direction is negative. This contradiction proves the Lemma.

Lemma 2.8. For every $(\varepsilon, k, L)$ we can find $\Gamma$ so that any solution in $\mathcal{M}_{1}$ with $R_{M A X} \geq \Gamma$ has an $(\varepsilon, k, L)$ neck at $t=1$.

Proof. Assume not. Pick a sequence of solutions in $\mathcal{M}_{1}$ with $R_{\text {MAX }} \rightarrow \infty$ but so that none of them has any $(\varepsilon, k, L)$ neck at $t=1$. Pick origins $P_{j}$ where $R_{P_{j}} \geq \frac{1}{2} R_{M A X}$ and $\lambda_{1, P_{j}} \geq \delta R_{M A X}$ by the previous Lemma. Dilate the solutions so $R_{\text {MAX }}=1$ and take a limit. We have to dilate and translate in time so the final time $t=1$ becomes $t=0$ and the solutions exist on an interval $\tau_{j} \leq t \leq 0$ with $\tau_{j} \rightarrow-\infty$. Then we can find a convergent subsequence using Theorem 25.1 in [10]. The limit will be an ancient solution on $-\infty<t \leq 0$ with bounded curvature and strictly positive curvature operator and satisfy $a_{3} \leq \Psi a_{1}$ and $c_{3} \leq \Psi c_{1}$ and $b_{3}^{2}<a_{1} c_{1}$. Such a solution must have an arbitrarily necklike end by the following Theorem E3.3, so each of the approaching solutions far enough out in the sequence has as good a neck as we like. This is a contradiction. It follows from our theorem in appendix $\mathrm{G}$ replacing the Schoenfliess conjecture that the $(\varepsilon, k, L)$ neck bounds a differentiable ball $B^{4}$. This will complete the proof of Theorem once we establish Theorem E3.3. 


\subsection{Ancient Solutions with Necklike Ends.}

Recall from section 19 in [10] the asymptotic scalar curvature ratio

$$
A=\limsup _{s \rightarrow \infty} R s^{2}
$$

where $s$ is the distance from some origin. The definition is independent of the choice of the origin and invariant under dilation. By Theorem 19.1 of that paper, if we have an ancient solution to the Ricci Flow complete with bounded curvature and positive curvature operator, $A$ is constant in time.

We say a complete Riemannian manifold with one topological end has an arbitrarily necklike end if for every $(\varepsilon, k, L)$ we can find an $(\varepsilon, k, L)$ neck in the manifold.

Theorem 3.1. Suppose we have an ancient solution to the Ricci Flow on $-\infty<t \leq 0$ which is complete with bounded curvature and positive curvature operator, and satisfies the pinching conditions

$$
a_{3} \leq \Psi a_{1} \quad \text { and } \quad c_{3} \leq \Psi c_{1} \text { and } b_{3}^{2} \leq a_{1} c_{1} .
$$

Then the solution has an arbitrarily necklike end if and only if $A=\infty$.

Proof. Suppose we have an arbitrarily necklike end. If $\varepsilon$ and $k$ are good, the scalar curvature $R$ in the neck is nearly $1 / r^{2}$ where $r$ is the average radius, and the length of the neck is nearly $L r$. The distance $s$ of some point on the boundary of the neck is nearly $L r / 2$ from the center, so $R s^{2}$ is nearly $L^{2} / 4$. Taking a sequence of necks with $L \rightarrow \infty$ we see $R s^{2}$ is unbounded, so $A=\infty$.

Conversely, suppose $A=\infty$. We can argue along the lines of the dimension reduction procedure of section 22 in [10]. By Lemma 22.2 of that paper, we can choose a sequence of points $P_{j}$ at time $t=0$ and radii $r_{j}$ and numbers $\delta_{j} \rightarrow 0$ such that

(a) $R(P, 0) \leq\left(1+\delta_{j}\right) R\left(P_{j}, 0\right)$ for all $P$ in the ball of radius $r_{j}$ around $P_{j}$ at $t=0$

(b) $r_{j}^{2} R\left(P_{j}, 0\right) \rightarrow \infty$

(c) if $s_{j}$ is the distance of $P_{j}$ from the origin at time 0 , then $s_{j} / r_{j} \rightarrow \infty$

(d) the balls in (a) are disjoint.

Let $\lambda_{j}$ be the smallest eigenvalue of the curvature operator at $P_{j}$ at time $t=0$.

Lemma 3.2. We have $\lambda_{j} / R\left(P_{j}, 0\right) \rightarrow 0$. 
Proof. Suppose not, so that $\lambda_{j} / R\left(P_{j}, 0\right) \geq \varepsilon>0$ for an infinite subsequence. The bound on $R$ in the ball of radius $r_{j}$ around $P_{j}$ at $t=0$ extends for time $t \leq 0$ by the Harnack estimate [6], and this gives interior derivative bounds. Since $r_{j}^{2} R\left(P_{j}, 0\right) \rightarrow \infty$ it will be at least 1 for a subsequence. Dilate the solutions to make $R\left(P_{j}, 0\right)$ become 1 , take $P_{j}$ to be the origins, and take a local limit of the dilated solutions in a ball of radius 1 for time $-1 \leq t \leq 0$ using geodesic coordinates (see [8]). We then have the smallest eigenvalue $\lambda \geq \varepsilon>0$ at the origin at time 0 . Since the solutions have curvature operator $R m \geq 0$, so does the limit.

Suppose $R m$ has a zero eigenvalue in the interior of the ball for the limit. Then the strong maximum principle would contradict $\lambda>0$ at the origin. This means that we can find $\delta>0$ such that $\lambda \geq \delta$ in the ball of radius $1 / 2$ around the origin at $t=0$. But this means that for large $j$ we have $\lambda \geq(\delta / 2) R\left(P_{j}, 0\right)$ in the ball of radius $r_{j} / 2$ around $P_{j}$ at $t=0$ in the sequence. Hence there is a $\beta>0$ such that each $P_{j}$ is the center of a curvature $\beta$-bump of radius $r_{j} / 2$. Since $s_{j} / r_{j} \rightarrow \infty$, for any $\lambda<\infty$ we can find a subsequence of disjoint $\lambda$-remote curvature $\beta$-bumps. This contradicts Theorem 21.6 in [10]. Hence $\lambda_{j} / R\left(P_{j}, 0\right) \rightarrow 0$.

Now we finish the proof of the Theorem as in section C5. We claim that for any $\left(\varepsilon^{\prime}, k^{\prime}, L^{\prime}\right)$ the points $P_{j}$ for large enough $j$ are the centers of $\left(\varepsilon^{\prime}, k^{\prime}, L^{\prime}\right)$ curvature necks. To see this, take geodesic tubes out of the $P_{j}$ for any length $\ell R\left(P_{j}, 0\right)^{1 / 2}$ in any direction ending in points $Q_{j}$ and still control the curvature. Since $r_{j}^{2} R\left(P_{j}, 0\right) \rightarrow \infty$ we can always go out a length $\ell R\left(P_{j}, 0\right)^{1 / 2}$ for large $j$ and still control the curvature. Make $P_{j}$ the origin and the directions the same, dilate so $R\left(P_{j}, 0\right)$ becomes 1 , and take a local limit along the geodesic tube. The limit still has non-negative curvature operator and satisfies the pinching conditions

$$
a_{3} \leq \Psi a_{1} \quad \text { and } \quad c_{3} \leq \Psi c_{1} \quad \text { and } \quad b_{3}^{2} \leq a_{1} c_{1}
$$

since they are dilation invariant. In the limit the curvature operator has a zero eigenvalue at the origin by the previous Lemma. Then as in Theorem C5.1 the holonomy reduces to $S o(3)$, the curvature operator matrix has $A=B=C$, and $b_{3}^{2} \leq a_{1} c_{1}$ forces $A=B=C$ to be a multiple of the identity. In dimension 3 if the curvature is a multiple of the identity at each point, it is constant. Hence the curvature in the limit is cylindrical. For large $j$ it will be $\left(\varepsilon^{\prime}, k^{\prime}\right)$ cylindrical in a ball of radius $\ell R\left(P_{j}, 0\right)^{1 / 2}$ for any $\ell$ we like. Taking $\ell \geq L^{\prime}$ establishes our claim that the curvature is $\left(\varepsilon^{\prime}, k^{\prime}, L^{\prime}\right)$ cylindrical. For any $(\varepsilon, k, L)$ we can pick $\left(\varepsilon^{\prime}, k^{\prime}, L^{\prime}\right)$ so that if the curvature is $\left(\varepsilon^{\prime}, k^{\prime}, L^{\prime}\right)$ cylindrical we have a geometric $(\varepsilon, k, L)$ neck. This proves the Theorem. 
The following result will complete the proof of the Enabling Theorem and hence also the Main Theorem A1.1.

Theorem 3.3. Given an ancient solution on $-\infty<t \leq 0$ to the Ricci Flow which is complete but not compact with bounded curvature and strictly positive curvature operator and satisfying the pinching estimates

$$
a_{3} \leq \Psi a_{1} \quad \text { and } \quad c_{3} \leq \Psi c_{1} \quad \text { and } \quad b_{3}^{2} \leq a_{1} c_{1}
$$

then for every $(\varepsilon, k, L)$ we can find an $(\varepsilon, k, L)$ neck somewhere in the solution at time $t=0$.

Proof. We consider two cases. The first is Type I where $R|t| \leq \Omega<\infty$. Since

$$
R=a_{1}+a_{2}+a_{3}=c_{1}+c_{2}+c_{3} \leq 3 a_{3} \text { or } 3 c_{3}
$$

we have uniform isotropic pinching. By Corollary B3.5 we can find points with necklike curvature, and use Theorem C5.1 (our hypotheses here are stronger) to find the desired neck.

The other case is Type II where

$$
\underset{t \rightarrow-\infty}{\rightarrow} \lim \sup R|t|=\infty
$$

Pick sequences $T_{j} \searrow-\infty$ and $\gamma_{j} \nearrow 1$, and then pick points $P_{j}$ and times $t_{j}$ so that $t_{j}>T_{j}$ and

$$
\left|t_{j}\right|\left(t_{j}-T_{j}\right) R\left(P_{j}, t_{j}\right) \geq \gamma_{j} \sup _{P, t}|t|\left(t-T_{j}\right) R(P, t) .
$$

Then translate in space so $P_{j}$ is the origin and in time so $t_{j}$ becomes time 0 , and dilate in space and time so $R(P, t)$ becomes 1 . Suppose time 0 dilates to become $\Omega_{j}$ and time $T_{j}$ dilates to becomes $-A_{j}$. Then

$$
\frac{1}{1 / A_{j}+1 / \Omega_{j}}=\frac{A_{j} \Omega_{j}}{A_{j}+\Omega_{j}}=\frac{\left|t_{j}\right|\left(t_{j}-T_{j}\right)}{T_{j}} R\left(P_{j}, t_{j}\right)
$$

by dilation invariance, and the last term goes to $\infty$ as $j \rightarrow \infty$ by our hypothesis that $|t| R$ is unbounded. Hence $A_{j} \rightarrow \infty$ and $\Omega_{j} \rightarrow \infty$.

If a subsequence of the lowest eigenvalues $\lambda_{1, j}$ of the curvature operators of the dilated solutions at the points $(0,0)$ (which were $\left(P_{j}, t_{j}\right)$ before we translate and dilate) have $\lambda_{1, j} \rightarrow 0$, we can find arbitrarily good necks in the solution, but at earlier times. If we find an $\left(\varepsilon_{p}, k_{p}, L_{p}\right)$ neck at time $t_{p}$, the 
asymptotic scalar curvature ratio $A$ at that time must be nearly $L_{p}^{2}$. But $A$ is constant in time and $L_{p}$ is arbitrarily large. Hence $A=\infty$. But then Theorem 3.1 implies we have arbitrarily good necks at time $t=0$ also.

If on the other hand the lowest eigenvalues $\lambda_{1, j}$ of the curvature operators of the dilated solutions are uniformly bounded away from 0 , we can control the injectivity radii by Theorem 25.1 of [10], and take a global limit. This limit will have strictly positive curvature operator, since $\lambda_{1}>0$ at the origin, and the scalar curvature $R$ will assume its maximum at the origin at time 0 by our choice of the $P_{j}$ and $t_{j}$. Then it is a Ricci soliton by [7], and by Theorem 20.2 in [10] such a soliton has asymptotic curvature ratio $A=\infty$. Hence the soliton limit has arbitrarily necklike ends. Then again we can find arbitrarily good necks in the original ancient solution at a sequence of times $t_{j} \rightarrow-\infty$. So $A$ is very large at these times, but $A$ is constant; hence $A=\infty$ in the ancient solution also and we can apply Theorem 3.1. Thiscompletes the Proof. 


\section{Appendices}

\section{Smoothing Functions.}

\subsection{Heat Flow Estimates on Tensors.}

We shall prove a smoothing theorem to deal with the following situation. We have a region in a manifold where we have a function $f_{0}$ and a one-form $H_{0}$ so that $D f_{0} \approx H_{0}$ and $D H_{0} \approx 0$. Then we would like to approximate $f_{0}$ by another function $f_{*}$ on a smaller region so that

$$
f_{*} \approx f_{0} \text { and } D f_{*} \approx H_{0} \text { and } D^{2} f_{*} \approx 0 .
$$

This requires smoothing since $f_{0}$ may not even have a second derivative.

Our technique for smoothing $f_{0}$ will be to solve a heat equation with Dirichlet boundary data, and use some fairly standard derivative estimates from the maximum principle. We shall also have to do the same with $H_{0}$. Of course one could probably do this also by writing down a convolution. However this becomes somewhat complicated in dealing with forms on a manifold, so the author has used a method with which he is more familiar.

We start by observing we can always take the boundary of a region to be smooth, up to a small error in distance. Of course we have no control over the extrinsic curvature of the boundary, but this will never enter our subsequent estimates. This is just a device to make things as smooth as possible to avoid technical questions when possible.

Lemma 1.1. Given a compact set $K$ inside an open set $U$ in a smooth manifold $M$, there exists a compact set $N$ with $K \subseteq N \subseteq U$ such that the boundary of $N$ is a smooth submanifold of $M$.

Proof. Choose a Riemannian metric on $M$. Cover $K$ with a finite number of open coordinate balls of radius at most $\rho$. If $\rho$ is small each coordinate ball is a smooth ball with a smooth boundary, and each ball lies well inside $U$. Call the balls $B_{1}, B_{2}, \ldots, B_{k}$. Let $s_{1}$ be the distance of a point outside $B_{1}$ to its boundary, and likewise for $s_{2}, \ldots, s_{k}$. There will be some $\delta>0$ such that each $s_{j}$ is smooth on $0 \leq s_{j} \leq 2 \delta$. By enlarging some of the radii of the balls slightly if necessary, we can guarantee they all meet transversally. Then we can find $\delta>0$ so that if some collection of balls, say $B_{1}, \ldots, B_{p}$, has 
$s_{1} \leq 2 \delta, \ldots, s_{p} \leq 2 \delta$ at a point then the vectors $D s_{1}, \ldots, D s_{p}$ are linearly independent at that point.

Choose a smooth function $\varphi(s)$ with $\varphi(s)=\delta$ for $0 \leq s \leq \delta$ and $\varphi(s)=$ $2 \delta$ for $s \leq 2 \delta$, while $\varphi^{\prime}(s)>0$ if $0 \leq s<2 \delta$ for all $s$. Let $\tilde{s}_{j}=\varphi\left(s_{j}\right)$. Then $\tilde{s}_{j}$ is smooth everywhere. Consider the product

$$
S=\tilde{s}_{1} \tilde{s}_{2} \ldots \tilde{s}_{k} .
$$

We let $N$ be the set where $S \leq \epsilon$ for some small $\epsilon>0$ to be chosen. Clearly $N$ contains $K$, and if $\epsilon$ is small $N$ is contained in $U$. If $\epsilon \leq \delta^{k}$ then when $S=\epsilon$ on the boundary of $N$, some function, say $\tilde{s}_{1}$, has $\tilde{s}_{1} \leq \delta$. Then $\tilde{s}_{1}=s_{1}$ and $D s_{1}$ is a unit vector, so $D \tilde{s}_{1} \neq 0$. Suppose $\tilde{s}_{1}, \tilde{s}_{2}, \ldots, \tilde{s}_{p} \leq 2 \delta$ while $\tilde{s}_{p+1}, \ldots, \tilde{s}_{k} \leq 2 \delta$. Since

$$
D \tilde{s}_{j}=\varphi^{\prime}\left(\tilde{s}_{j}\right) D s_{j}
$$

and $D s_{1}, \ldots D s_{p}$ are linearly independent, so are $D \tilde{s}_{1}, \ldots D \tilde{s}_{p}$; while $D \tilde{s}_{p+1}, \ldots, D \tilde{s}_{k}=0$ since $\varphi^{\prime}(s)=0$ for $s \geq 2 \delta$ by the product rule

$$
D S=D \tilde{s}_{1} \cdot \tilde{s}_{2} \ldots \tilde{s}_{k}+\tilde{s}_{1} \cdot D \tilde{s}_{2} \cdot \tilde{s}_{3} \ldots \tilde{s}_{k}+\ldots
$$

and we see that $D S \neq 0$ or $S=\epsilon$. This shows the boundary of $N$ is smooth, proving Lemma 1.1.

Let $N$ be a compact manifold with boundary. Choose a distance $s$ on $N$. Let $N_{s}$ denote the set of points whose distance from the boundary $\partial N$ is $s$ or more. We assume $N$ has a Riemannian metric whose sectional curvature tensor is bounded in absolute value by

$$
|R m| \leq 1 / s^{2}
$$

which is always true if $s$ is small. Let $P$ be a smooth tensor on $N$ evolving over time by a heat equation of the form

$$
\frac{\partial P}{\partial t}=\Delta P+L * P+Q
$$

where $L$ and $Q$ are some other tensors on $N$ and $L * P$ is a tensor contraction. We assume moreover that for some constant $B$ we have bounds

$$
|P| \leq B \text { and }|L| \leq 1 / s^{2} \text { and }|Q| \leq B / s^{2}
$$

on $N$ for $0 \leq t \leq \omega$. We also assume $s$ is so small that $s^{2} \leq \omega$. 
Theorem 1.2. Suppose in addition

$$
|P| \leq \delta B
$$

for some small $\delta \geq 0$ on $N$ at $t=0$. Then also

$$
|P| \leq\left(\delta+C t / s^{2}\right) B
$$

on $N_{s}$ for $0 \leq t \leq s^{2}$ for some constant $C$ depending only on the dimension.

Proof. Let

$$
p=e^{-t / s^{2}}(1+|P| / B)-(1+\delta) .
$$

Then $p$ is smooth for $P \neq 0$, and $P=0$ will not matter. Using our estimates we find that $p \leq 1$ on $N$ for $t \geq 0$ and $p \leq 0$ on $N$ at $t=0$. Moreover $p$ satisfies the inequality

$$
\frac{\partial p}{\partial t} \leq \Delta p
$$

on $N$ for $t \leq 0$, as we see from the estimates on $L$ and $Q$. We want to keep $p$ from growing too fast on $N_{s}$.

Take any point $X$ in $N_{s}$ and look at the exponential map at $X$ taking a ball of radius $s$ in the tangent space at $X$ into $N$. Since $|R m| \leq 1 / s^{2}$ this is a local cover. By reducing the original $s$ by a constant factor, we can get such a cover on a ball of radius many times $s$, and this will only scale the constant $C$ in our estimate. Then we can find harmonic coordinates on the ball of radius $s$ in the tangent space. By making $s$ small, these coordinates will be such that the metric is nearly the identity and its first derivatives are nearly zero. If $r$ is the Euclidean radius in the ball, we will have $\Delta r^{2}$ nearly equal to $2 n$; this gives

$$
\Delta r^{2} \leq C
$$

for a constant depending only on $n$. Let

$$
q=\frac{r^{2}+C t}{s^{2}}
$$

on the ball. Then $q \leq 0$ on the ball, and $q \geq 1$ on its boundary. Moreover

$$
\frac{\partial q}{\partial t} \geq \Delta q
$$


by the previous estimate. If we pull back the function $p$ to the ball, we find that $p \leq q$ by the maximum principle, since this holds at $t=0$ and on the boundary of the ball. This gives

$$
|P| \leq B\left[\delta+\left(e^{t / s^{2}}-1\right)+C B t / S^{2}\right]
$$

at $X$ since $q \leq C t / s^{2}$ there. Now

$$
e^{t / s^{2}}-1 \leq C t / s^{2}
$$

for $0 \leq t \leq s^{2}$, and we are done.

Corollary 1.3. If we only assume $P$ is in $L^{\infty}$ at $t=0$ with the same bounds, the conclusion still holds for the weak solution.

Proof. We can approximate $P$ by smooth functions at $t=0$ in the classical way using consolutions in local coordinates and patching together with a partition of unity. This gives us a sequence of smooth functions $P_{j}$ at $t=0$ with $P_{j} \rightarrow P$ weakly and

$$
\limsup _{j \rightarrow \infty} \sup _{x}\left|P_{j}(x, 0)\right| \leq \underset{x}{\operatorname{ess} \sup }|P(x, 0)|
$$

Solve the same linear equation with smooth coefficients for $t \geq 0$ using the $P_{j}$ as initial data. Then the solutions for the $P_{j}$ converge weakly to the solution $P$. Since the $P_{j}$ satisfy the bounds of the Theorem, so does $P$.

Now we give estimates on the first derivative. Suppose again that $P$ is smooth and

$$
\frac{\partial P}{\partial t}=\Delta P+L * P+Q
$$

on $0 \leq t \leq s^{2}$ and

$$
|P| \leq B \text { and }|L| \leq 1 / s^{2} \text { and }|Q| \leq B / s^{2} \text { and }|R m| \leq 1 / s^{2}
$$

and now suppose also that

$$
|D L| \leq 1 / s^{3} \text { and }|D \varphi| \leq B / s^{3} \text { and }|D R m| \leq 1 / s^{3} .
$$

Theorem 1.4. We have an estimate

$$
|D P| \leq C B / \sqrt{t}
$$


on $N_{s}$ for $0 \leq t \leq s^{2}$. If we assume in addition that

$$
|D P| \leq B / s
$$

at $t=0$ then we also have an estimate

$$
|D P| \leq C B / s
$$

for $0 \leq t \leq s^{2}$. Here $C$ denotes constants depending only on the dimension.

Proof. We let

$$
F=a\left(A B^{2}+|P|^{2}\right)|D P|^{2} / B^{4}
$$

where $a$ and $A$ are constants we can choose later. The derivative $D P$ will satisfy an equation of the form

$$
\frac{\partial}{\partial t} D P=\Delta D P+R m * D P+D R m * P+L * D P+D L * P+D Q
$$

and hence $F$ will satisfy an equation of the form

$$
\frac{\partial F}{\partial t}=\Delta F-\frac{2 a}{B^{4}}\left[|D P|^{4}+\left(A B^{2}+|P|^{2}\right)\left|D^{2} P\right|^{2}\right]+\frac{a E}{B^{4}}
$$

where $E$ is a sum of terms of the form

$$
\begin{aligned}
& E_{1}=P * D P * D P * D^{2} P \\
& E_{2}=P *(L * P+Q)|D P|^{2} \\
& E_{3}=\left(A B^{2}+|P|^{2}\right)[R m * D P+D R m * P+L * D P+D L * P+D Q] * D P .
\end{aligned}
$$

Our estimates give

$$
\begin{aligned}
& \left|E_{1}\right| \leq C B|D P|^{2}\left|D^{2} P\right| \\
& \left|E_{2}\right| \leq C B^{2}|D P|^{2} / s^{2} \\
& \left|E_{3}\right| \leq C B^{2}|D P|^{2} / s^{2}+C B^{3}|D P| / s^{3}
\end{aligned}
$$

and we can use the negative terms to get

$$
\frac{\partial F}{\partial t} \leq \Delta F-F^{2}+C / s^{4}
$$

by making $A$ large enough to bound $E_{1}$, taking care of $E_{2}$ and $E_{3}$, and making $a$ small enough that the term in $|D P|^{4}$ bounds $F^{2}$. If we let

$$
\widetilde{F}=F-C t / s^{4}
$$


then when $\widetilde{F} \geq 0$ (which is all we need) we have

$$
\frac{\partial \widetilde{F}}{\partial t} \leq \Delta \widetilde{F}-\widetilde{F}^{2}
$$

This is our basic differential inequality.

To estimate $\widetilde{F}$ we again construct a comparison function in a harmonic coordinate ball on a local cover. If $r$ is the Euclidean radius in the ball, we have

$$
\left|D r^{2}\right| \leq C s \text { and }\left|\Delta r^{2}\right| \leq C
$$

as before. Then we let

$$
h=\frac{1}{t}+\frac{A s^{2}}{\left(s^{2}-r^{2}\right)^{2}}
$$

where $A$ is a constant we can choose later. Since

$$
\frac{\partial h}{\partial t}=-\frac{1}{t^{2}} \quad \text { and } \quad \Delta h \leq \frac{C A s^{4}}{\left(s^{2}-r^{2}\right)^{4}},
$$

if $A$ is large compared to $C$ we get

$$
\frac{\partial h}{\partial t} \geq \Delta h-h^{2}
$$

and $h \rightarrow \infty$ if $t \rightarrow 0$ or $r \rightarrow s$. Therefore by the maximum principle $\widetilde{F} \leq h$. This gives the bound (i) in the general case.

If we also assume $|D P| \leq B / s$ at $t=0$, we get a bound

$$
\widetilde{F} \leq C / s^{2} \text { at } t=0
$$

and we can take the simpler comparison function

$$
h=\frac{A s^{2}}{\left(s^{2}-r^{2}\right)^{2}}
$$

with $A$ suitably large compared to the constants $C$. Then we still have

$$
\frac{\partial h}{\partial t} \geq \Delta h-h^{2}
$$

and $\widetilde{F} \leq h$ at $t=0$, while $h \rightarrow \infty$ as $r \rightarrow s$. This makes $\widetilde{F} \leq h$ for $t \geq 0$, which gives the bound (ii).

Corollary 1.5. If we only assume $P$ is in $L^{\infty}$ with the same bounds, (i) still holds. If we only assume $P$ is Lipschitz continuous with the same bounds, (ii) holds. 
Proof. The result for (i) is the same proof as Corollary 1.3 before. The result for (ii) is similar. If $P$ is Lipschitz, the sequence of smooth approximations $P_{j}$ at $t=0$ will have

$$
\limsup _{j \rightarrow \infty} \sup _{x}\left|P_{j}(x, 0)\right| \leq \sup _{x}|P(x, 0)|
$$

and for the covariant derivative

$$
\limsup _{j \rightarrow \infty} \sup _{x}\left|D P_{j}(x, 0)\right| \leq \limsup _{d(x, y) \rightarrow 0}|P(x, 0)-P(y, 0)| / d(x, y) .
$$

Hence we can replace the bound on $D P$ at $t=0$ by the Lipschitz seminorm.

\subsection{The Smoothing Procedure.}

Now we prove our smoothing result.

Theorem 2.1 Smoothing Theorem. In any dimension $n$, for every $\epsilon>$ 0 there exists a $\delta>0$ depending only on $\epsilon$ and $n$ with the following property. If $N$ is a smooth manifold with boundary $\partial N$ of dimension $n, f_{0}$ is a Lipschitz continuous function and $H_{0}$ is a Lipschitz continuous one-form on $N$, $s$ is a distance and $B$ is a constant such that

$$
|R m| \leq 1 / s^{2} \text { and }|D R m| \leq 1 / s^{3} \text { and }\left|D^{2} R m\right| \leq 1 / s^{4}
$$

and all of $N$, and

$$
\left|f_{0}\right| \leq B \text { and }\left|H_{0}\right| \leq B / s
$$

on all of $N$, and

$$
\left|D f_{0}-H_{0}\right| \leq \delta B / s \text { and }\left|D H_{0}\right| \leq \delta B / s^{2}
$$

almost everywhere on $N$, then we can find a smooth function $f_{*}$ on the interior of $N$ such that

$$
\left|f_{*}-f_{0}\right| \leq \epsilon B \text { and }\left|D f_{*}-H_{0}\right| \leq \epsilon B / s \quad \text { and } \quad\left|D^{2} f_{*}\right| \leq \epsilon B / s^{2}
$$

at every point of $N$ whose distance from the boundary $\partial N$ is at least $s$. 
Proof. We shall start with the given value $f_{0}$, and construct a one-parameter family of functions $f_{t}$ for $t \leq 0$ by solving the heat equation

$$
\frac{\partial f}{\partial t}=\Delta f
$$

on $N$ with initial value and boundary value conditions $f=f_{0}$ at $t=0$ and on $\partial N$. The resulting solution is smooth in the interior of $N$ for $t>0$. We will take our smoothing $f_{*}$ to be the value of the solution $f_{t}$ at a suitable value $t_{*}$ of $t$.

Lemma 2.2. For all $t \geq 0$ we have $|f| \leq B$ on all of $N$.

Proof. This follows from the maximum principle since $|f| \leq B$ at $t=0$ and on $\partial N$.

Lemma 2.3. We have an estimate

$$
|D f| \leq C B / s
$$

at all points at distance at least $s$ from $\partial N$ for $0 \leq t \leq s^{2}$.

Proof. We can apply our interior derivative estimate to $D f$. At $t=0$ we have

$$
|D f| \leq|D f-H|+|H| \leq(\delta+1) B / s
$$

and we can take $\delta \leq 1$. We also have bounds on $R m$ and $D R m$, and this is all we need to use Theorem 1.4 (ii).

Lemma 2.4. We have an estimate

$$
\left|D^{2} f\right| \leq C B / s \sqrt{t}
$$

at distance at least $s$ from $\partial N$ for $0<t \leq s^{2}$.

Proof. Let $N_{s}$ denote the points at distance $s$ from $\partial N$. Then

$$
N_{s}=\left(N_{s / 2}\right)_{s / 2}
$$


The previous Lemma gives an estimate of the same form on $N_{s / 2}$ with a larger constant $C$. We use this as our bound on $D f$ when we apply the interior derivative estimate to $D^{2} f$. Now $D f$ satisfies an equation

$$
\frac{\partial}{\partial t} D_{i} f=\Delta D_{i} f-2 R_{i j} D_{j} f
$$

which has the form

$$
\frac{\partial}{\partial t} D f=\Delta D f+R m * D f
$$

and we have bounds on $R m$ and $D R m$. Then we can use Theorem 1.4(i).

Corollary 2.5. We have an estimate

$$
\left|f_{*}-f_{0}\right| \leq C B \sqrt{t} / s
$$

at distance $s$ from the boundary for $0 \leq t \leq s^{2}$.

Proof. We use

$$
\frac{\partial f}{\partial t}=\Delta f \quad \text { and } \quad|\Delta f| \leq C\left|D^{2} f\right|
$$

and integrate the previous estimate from 0 to $t$. Note that although $1 / \sqrt{t} \rightarrow$ $\infty$ as $g \rightarrow 0$, its integral is bounded by $\sqrt{t}$.

For the first inequality in the theorem to hold, we need to make

$$
\left|f_{*}-f_{0}\right| \leq \varepsilon B .
$$

We can accomplish this by Corollary 2.5 by making

$$
t_{*} \leq(\epsilon s / C)^{2} .
$$

This is one condition we must meet.

To make further estimates, we construct a one-parameter family of oneforms $H_{t}$ for $t \geq 0$ by solving the heat equation

$$
\frac{\partial H}{\partial t}=\Delta_{B} H
$$

where $\Delta_{B}$ is the Bochner Laplacian on one-forms

$$
\Delta_{B} H=\left(d d^{*}+d^{*} d\right) H
$$


given in coordinates by

$$
\Delta_{B} H_{k}=\Delta H_{k}-R_{k \ell} H_{\ell}
$$

where $\Delta$ is the naive Laplacian

$$
\Delta H_{k}=g^{i j} D_{i} D_{j} H_{k} .
$$

Note that $D f$ satisfies the same equation

$$
\frac{\partial}{\partial t} D f=\Delta_{B} D f
$$

The equation has the form

$$
\frac{\partial}{\partial t} D f=\Delta_{B} D f
$$

The equation has the form

$$
\frac{\partial}{\partial t} H=\Delta H+R m * H
$$

which is all we use for our estimates. We impose the initial value and boundary value conditions

$$
H=H_{0} \quad \text { at } t=0 \text { and on } \partial N \text {. }
$$

Lemma 2.6. For $0 \leq t \leq s^{2}$ we have an estimate

$$
|H| \leq C B / s
$$

on all of $N$ for some constant $C$.

Proof. The estimate holds at $t=0$ and on $\partial N$. The length squared of $H$ satisfies an equation of the form

$$
\frac{\partial}{\partial t}|H|^{2}=\Delta|H|^{2}-2|D H|^{2}+R m * H * H
$$

which gives an inequality

$$
\frac{\partial}{\partial t}|H|^{2} \leq \Delta|H|^{2}+C|H|^{2} / s^{2} .
$$


The maximum principle can be applied to

$$
h=e^{-C t / s^{2}}|H|^{2}
$$

since

$$
\frac{\partial h}{\partial t} \leq \Delta h
$$

and $h \leq B / s$ at $t=0$ and on $\partial N$. This gives $h \leq B^{2} / s^{2}$ for $t \geq 0$ on $N$, and for $0 \leq t \leq s^{2}$ we get $|H|^{2} \leq C B^{2} / s^{2}$.

Lemma 2.7. We have an estimate

$$
|D H| \leq C B / s^{2}
$$

at all points at distance at least $s$ from $\partial N$ for $0 \leq t \leq s^{2}$.

Proof. We apply the interior derivative estimate to $D H$. At $t=0$ we use

$$
|D H| \leq \delta B / s^{2}
$$

and take $\delta \leq 1$, since it can't help to make it small because there are other terms in the answer without $\delta$. We can apply Theorem 1.4(ii) to get the result.

Lemma 2.8. We have an estimate

$$
\left|D^{2} H\right| \leq C B / s^{2} \sqrt{t}
$$

at all points at distance at least $s$ from $\partial N$ for $0 \leq t \leq s^{2}$.

Proof. First we use the previous result to estimate $|D H|$ not just on $N_{s}$ but on the larger set $N_{s / 2}$, and then use this to estimate $\left|D^{2} H\right|$ on $N_{s}$. The tensor $D H$ satisfies an evolution equation of the form

$$
\frac{\partial}{\partial t} D H=\Delta D H+R m * D H+D R m * H
$$

and we have bounds on $R m$ and $D R m$ and $D^{2} R m$ and $H$. and $D H$. We can use Theorem 1.3(i). 
Corollary 2.9. We have an estimate

$$
\left|H_{t}-H_{0}\right| \leq C B \sqrt{t} / s^{2}
$$

at all points at distance at least $s$ for $\partial N$ for $0 \leq t \leq s^{2}$.

Proof. We use the equation for $\partial H / \partial t$ and integrate the previous bound from 0 to $t$.

Next consider the tensor

$$
J=D f-H
$$

which also satisfies the equation

$$
\frac{\partial J}{\partial t}=\Delta_{B} J
$$

which has the form

$$
\frac{\partial J}{\partial t}=\Delta J+R m * J
$$

Using our estimates on $D f$ and $H$ we have a bound

$$
|J| \leq C B / s
$$

on $N_{s}$ for $t \geq 0$, and hence a similar bound on $N_{s / 2}$. By our hypotheses we also have a bound

$$
|J| \leq \delta B / s
$$

on $N_{s}$ at $t=0$.

Lemma 2.10. We have a bound

$$
|J| \leq\left(\delta+C t / s^{2}\right) B / s
$$

at all points at distance at least $s$ from $\partial N$ for $0 \leq t \leq s^{2}$.

Proof. We apply our bound in Theorem 1.2 to $J$ on $N_{s / 2}$.

Now observe that

$$
D f_{t}-H_{t}=J_{t}+\left(H_{t}-H_{0}\right)
$$


and hence on $N_{s}$ we have an estimate

$$
\left|D f_{t}-H_{t}\right| \leq(\delta+C \sqrt{t} / s) B / s
$$

for $0 \leq t \leq s^{2}$ using Lemma 2.10 and Corollary 2.9 and noting $t / s^{2} \leq \sqrt{t} / s$.

We can then make

$$
\left|D f_{*}-H_{*}\right| \leq \epsilon B / s
$$

by choosing $\delta \leq \epsilon / 2$ and $t_{*} \leq(\epsilon s / 2 C)^{2}$.

Lemma 2.11. We have a bound

$$
|D H| \leq\left(\delta+C t / s^{2}\right) B / s^{2}
$$

at all points at distance $s$ from $\partial N$ for $0 \leq t \leq s^{2}$.

Proof. We have $|D H| \leq C B / s^{2}$ on $N_{s / 2}$ for $0 \leq t \leq s^{2}$ from Lemma 7 and $|D H| \leq \delta B / s^{2}$ on $N$ at $t=0$ from the hypotheses. We can then apply Theorem 1 to $D H$ on $N_{s}$.

Lemma 2.12. We have a bound

$$
|D J| \leq C\left(\delta+t / s^{2}\right) B / s \sqrt{t}
$$

at all points at distance at least $s$ from $\partial N$ for $0 \leq t \leq s$ ?

Proof. We use the evolution equation for $J$ and the bound on $J$ from Lemma 10, which will also hold on $N_{s / 2}$ in the same form. We can then apply Theorem 1.4(i).

We are now in a position to estimate $D^{2} f_{t}$ using

$$
D^{2} f_{t}=D J_{t}+D H_{t}
$$

to get

$$
\left|D^{2} f_{t}\right| \leq C\left(\delta+t / s^{2}\right) B / s \sqrt{t}
$$

on $N_{s}$ for $0 \leq t \leq s^{2}$ (using $1 / s \leq 1 / \sqrt{t}$ in the estimate for $D H$. We can now make

$$
\left|D^{2} f_{*}\right| \leq \epsilon B / s^{2}
$$

by first making

$$
t_{*} \leq(\epsilon s / 2 C)^{2}
$$


and then making

$$
\delta \leq \epsilon \sqrt{t}_{*} / 2 C s
$$

Since these are compatible with our previous restrictions we are done. This proves the smoothing theorem.

\section{A Replacement for Schoenfliess Conjecture.}

We use the following result, which is much easier than the Conjecture.

Theorem 1.1. There exists $(\epsilon, k, L)$ with the following property. If we have a complete Riemannian manifold $M^{4}$ with a metric of strictly positive sectional curvature $K>0$, and if $M^{4}$ contains an $(\epsilon, k, L)$ neck, then its center sphere $S^{3}$ bounds a differentiable ball $B^{4}$.

Remark. A complete Riemannian manifold $M^{n}$ with strictly positive sectional curvature is diffeomorphic to $R^{n}$, and a differentiable sphere $S^{n-1}$ in $R^{n}$ is known to bound a differentiable ball $B^{n}$ in all dimensions except $n=4$ by the Schoenfliess conjecture. But our proof works in all dimensions.

Proof. The standard proof that $M^{n}$ is diffeomorphic to $R^{n}$ above is to construct a strictly convex Lipschitz Busemann exhaustion function $b$, and smooth $b$ a little to get a $C^{\infty}$ strictly convex exhaustion function $\tilde{b}$. Since $\tilde{b}$ has a single central point which is a minimum, the result follows by Morse Theory. Of course every sublevel set $\tilde{b} \leq C$ is a differentiable ball $B^{n}$. We shall show that a level set $\tilde{b}=C$ near the middle of a good long neck is transverse to the foliation of the neck $S^{3} \times B^{1}$ by lines $\{P\} \times B^{1}$, in fact being nearly perpendicular. It is then easy to see that the sublevel set $\tilde{b} \leq C$, which is a differentiable ball, is diffeomorphic to the ball inside the center sphere of the neck.

For simplicity, we scale the metric so the radius of the standard metric on $S^{3}$ is 1 , and the metric on $M^{4}$ is $(\epsilon, k, L)$ close to the product $S^{3} \times[0, L]$. Let $f$ be the function on the neck corresponding to the coordinate in $[0, L]$. We also take a Busemann function $b$ on $M^{4}$ from the standard construction (see Cheeger and Ebin[1]).

Lemma 2. There exists a function $b$ on $M^{4}$ with the following properties.

- (i) b is proper and bounded below

- (ii) b is Lipschitz continuous; $|b(Y)-b(X)| \leq d(X, Y)$ 
- (iii) $b$ is strictly convex (in the sense that the composition of $b$ with any geodesic $s: R^{1} \rightarrow M^{4}$ is a strictly convex function of the arc length $s$ )

- (iv) for any $r \leq s$, the compact strictly convex sublevel set $B_{r}=\{b \leq$ $r\}$ is exactly the set of all points in $B_{s}$ whose distance from the boundary of $B_{s}$ is at least $s$.

Since $b$ is strictly convex, if we smooth it a little (using smoothing operators as in Appendix F) it will still be strictly convex. We shall show that near the middle of the neck $b$ is close to $f$ (if we shift $b$ by a constant) both in the supremum sense $|b-f| \leq \delta$ and in the Lipschitz sense

$$
|[b(Y)-f(Y)]-[b(X)-f(X)]| \leq \delta d(X, Y) .
$$

Then if we smooth $b$ a little to $\tilde{b}$, and smooth $f$ by the same process to $\tilde{f}$, then $\tilde{b}-\tilde{f}$ will still be small in the Lipschitz sense (see Appendix $\mathrm{F}$ again), and $\tilde{f}$ will still be close to $f$ in the Lipschitz sense. This suffices to make the level sets of $\tilde{b}$ near the middle of the cylinder almost perpendicular to the lines of the cylinder, as desired. It remains to show $b$ is close to $f$ as claimed.

Lemma 4. For every $\delta>0$ and every fraction $c>0$ we can find $(\epsilon, k, L)$ so that once we are the fraction $c$ of the way from the far end of the cylinder we have $|b-f| \leq \delta$.

Proof. First we shift $b$ by a constant so that on the far end of the cylinder, where $f=L$, the minimum value of $b$ is exactly $L$, occurring at some point $P$. Consider any point $X$ in the cylinder on the sphere where $f(X)=L-\ell$. The value of $b$ at $X$ is given by

$$
b(X)=L-d(X,\{b=L\}) .
$$

Since $P \in\{b=L\}$, we have

$$
b(X) \geq L-d(X, P) .
$$

In the product metric $\bar{d}$

$$
\bar{d}(X, P)^{2} \leq \ell^{2}+\pi^{2}
$$

and this makes

$$
\bar{d}(X, P) \leq \ell+\frac{\pi}{2 \ell}
$$


while in our metric the distance will not be much more if $(\epsilon, k, L)$ is good. If we take $\ell$ large compared to $\delta$ we can make

$$
d(X, P) \leq \ell+\delta
$$

and get

$$
b(X) \geq L-\ell-\delta=f(X)-\delta .
$$

On the other hand, since the set $\{b=L\}$ lies on or beyond the far end of the cylinder, the distance from $X$ to $\{b=L\}$ is at least the distance to the far end. In the product metric this distance is $\ell$, and we can make it at least $\ell-\delta$ in our metric if $(\epsilon, k, L)$ is good. Then

$$
d(X, P) \geq \ell-\delta
$$

gives

$$
b(X) \leq L-\ell+\delta=f(X)+\delta
$$

and we are done.

Lemma 5. For every $\delta>0$ and every fraction $c>0$ we can find $(\epsilon, k, L)$ so that once we are the fraction $c$ from the far end of the cylinder we have a Lipschitz estimate

$$
|[b(Y)-f(Y)]-[b(X)-f(X)]| \leq \delta d(X, Y) .
$$

Proof. By the previous Lemma, we can afford to assume that at the far end of the cylinder we already have $|b-f| \leq \delta$. Choose $M$ so the level set $\{b=M\}$ is between the level spheres $\{f=L\}$ and $\{f=L-\delta\}$, and with $L-\delta \leq M \leq L$. Then take points $X$ and $Y$ near each other and well down the cylinder.

Let $P$ be the point on the level set $\{b=M\}$ closest to $X$. Then

$$
b(X)=M-d(X, P)
$$

while for any $Y$ we have

$$
b(Y) \leq M-d(Y, P)
$$

and this gives

$$
b(Y)-b(X) \leq d(X, P)-d(Y, P) .
$$


Choose minimal geodesics $X Y, X P$, and $Y P$, and let $\theta$ be the angle between $X Y$ and $X P$. When $X$ is far down the cylinder from $P$, the tangent vector to any minimal geodesic $X P$ must be close to the gradient vector $D f(X)$. In fact we can make the angle between them as small as we like by making $(\epsilon, k, L)$ good and making $X P$ a fraction of $L$. Then since $f$ is smooth we can make

$$
|f(Y)-f(X)-d(X, Y) \cos \theta| \leq \delta d(X, Y) / 2
$$

for $Y$ close to $X$. Now since $K>0$, Toponogov's Theorem (see [1] again) allows us to compare to a Euclidean triangle with sides $d(X, Y)$ and $d(X, P)$ and angle $\theta$ between them to conclude

$$
d(Y, P)^{2} \leq d(X, P)^{2}+d(X, Y)^{2}-2 d(X, P) d(X, Y) \cos \theta
$$

by the law of cosines. We rewrite this as

$$
d(Y, P)^{2} \leq[d(X, P)-d(X, Y) \cos \theta]^{2}+d(X, Y)^{2} \sin ^{2} \theta .
$$

Now we use $\sin \theta \leq 1$ and

$$
\sqrt{a^{2}+b^{2}} \leq a+\frac{b^{2}}{2 a}
$$

and $\cos \theta \leq 1$ in the denominator to get

$$
d(Y, P) \leq d(X, P)-d(X, Y) \cos \theta+\frac{d(X, Y)^{2}}{d(X, P)-d(X, Y)} .
$$

If $P$ is far down the cylinder and $X$ is close to $Y$ we can make the last term smaller than $\delta d(X, Y) / 2$. This gives

$$
b(Y)-f(Y) \leq b(X)-f(X)+\delta d(X, Y)
$$

and since $X$ and $Y$ are interchangeable we are done. This completes the proof of Theorem 1 .

\section{References.}

[1] J. Cheeger and D. Ebin, Comparison theorems in Riemannian geometry.

[2] R. S. Hamilton (1982), Three-manifolds with positive Ricci curvature, J. Differential Geom. 17, 255-306. 
[3] R. S. Hamilton (1986), Four-manifolds with positive curvature operator, J. Differential

Geom. 24, 153-179.

[4] R. S. Hamilton (1988), The Ricci flow on surfaces, Contemporary Mathematics $71,237-261$.

[5] R. S. Hamilton (1989), Lecture Notes on Heat Equations in Geometry, Honolulu, Hawaii.

[6] R. S. Hamilton (1993), The Harnack estimate for the Ricci flow, J. Differential Geom. 37, 225-243.

[7] R. S. Hamilton (1993), Eternal solutions to the Ricci flow, J. Differential Geom. 38, 113-126.

[8] R. S. Hamilton, A compactness property for solutions of the Ricci flow, to appear, Am. J. Math.

[9] R.S. Hamilton (1993), A matrix Harnack estimate for the heat equation. Comm. Anal. Geom. Vol.1, No.1, 113-126.

[10] R. S. Hamilton, The formation of singularities in the Ricci Flow, preprint UCSD.

[11] G. Huisken (1985), Ricci deformation of the metric on a riemannian manifold, J. Differential Geom. 21.

[12] J. Jost and H. Karcher (1982), Geom. Meth. zur gewinnung für harmonische Abbilding, Manuscripta Math. 40, 27-77.

[13] M. Micallef and J.D. Moore (1988), Minimal two-spheres and the topology ofmanifolds with positive curvature on totally isotropictwo-planes, Ann. of Math. (2) 127 No.1, 199-227.

RECEIVED NOVEMBER 3, 1994.

DEPARTMENT OF MATHEMATICS

UNIVERSiTy of CALIForNia, SAN DiEgo

LA JOLLA, CA 92093 